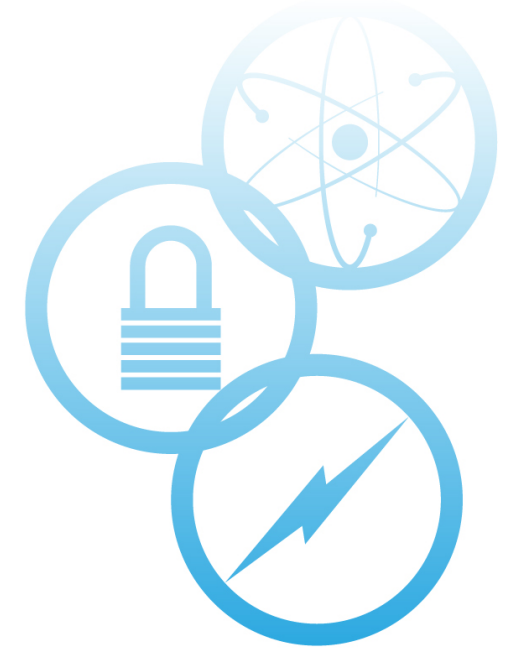

\title{
Increasing the Usability of Sockeye: Implementing Customer-Driven Modeling Improvements, Features, and Documentation of Sockeye
}

\section{March 2021}

J. E. Hansel ${ }^{1}$

L. C. Charlot ${ }^{1}$

${ }^{1}$ Idaho National Laboratory

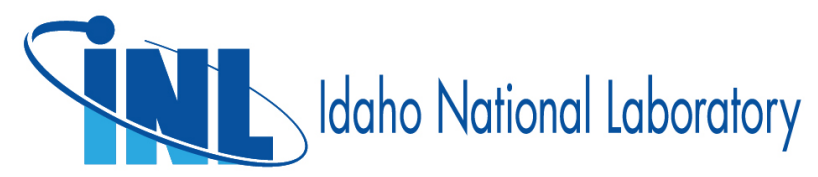




\section{DISCLAIMER}

This information was prepared as an account of work sponsored by an agency of the U.S. Government. Neither the U.S. Government nor any agency thereof, nor any of their employees, makes any warranty, expressed or implied, or assumes any legal liability or responsibility for the accuracy, completeness, or usefulness, of any information, apparatus, product, or process disclosed, or represents that its use would not infringe privately owned rights. References herein to any specific commercial product, process, or service by trade name, trade mark, manufacturer, or otherwise, does not necessarily constitute or imply its endorsement, recommendation, or favoring by the U.S. Government or any agency thereof. The views and opinions of authors expressed herein do not necessarily state or reflect those of the U.S. Government or any agency thereof. 


\title{
Increasing the Usability of Sockeye: Implementing Customer-Driven Modeling Improvements, Features, and Documentation of Sockeye
}

\author{
J. E. Hansel ${ }^{1}$ \\ L. C. Charlot $^{1}$ \\ ${ }^{1}$ Idaho National Laboratory
}

March 2021

\begin{abstract}
Idaho National Laboratory
Computational Frameworks Department

Idaho Falls, Idaho 83415
\end{abstract}

http://www.inl.gov

Prepared for the

U.S. Department of Energy

Office of Nuclear Energy

Under DOE Idaho Operations Office

Contract DE-AC07-05ID14517 
Page intentionally left blank 


\section{SUMMARY}

Numerous nuclear microreactor designs are currently under development, with an ambitious timeline that includes demonstrations within the next five years. The key attribute of these reactors is their relatively compact size, which makes them able to be factory-assembled and shipped to site, rather than requiring large-scale construction onsite. Many of these reactors employ heat pipe technology for cooling and heat transfer. Heat pipes have been used for decades in a wide variety of applications because of their extreme thermal efficiency, compactness, manufacturing simplicity, and reliability. While reliable, heat pipes have limitations on their heat throughput, due to a variety of thermal hydraulic phenomena. These limits motivate the need for modeling and simulation, as traditional methods of analysis are largely limited to steady-state analysis and usually employ additional, restrictive assumptions. To support this effort, the Nuclear Energy Advanced Modeling and Simulation (NEAMS) program began development of Sockeye, an engineeringscale heat pipe tool to be used for the design and safety analysis of nuclear microreactors. Sockeye is built on the Multiphysics Object-Oriented Simulation Environment (MOOSE) framework, which allows for seamless multiphysics coupling with other NEAMS tools for full microreactor modeling.

Sockeye development is largely driven by the needs of key stakeholders, such as Westinghouse Electric Company. Stakeholders have provided ample feedback and numerous discussions on their functional requirements, which led to the identification of a number of tasks, which this report discusses. These tasks include new capabilities, model improvements, user documentation and examples, and verification and validation.

New capabilities were implemented to increase the flexibility of the existing model and provide new tools to facilitate heat pipe design exploration, allowing users to quickly estimate performance from design parameters, before engaging in a more comprehensive design evaluation via simulation. Flexibility improvements made to the existing heat pipe model include the ability to provide a general working fluid distribution, the ability to provide non-constant permeability for the wick, and the ability to provide a closure relationship for the interfacial curvature. An alternative heat pipe model provides users with a simple, robust surrogate model to complement Sockeye's higher fidelity model. Design utilities were created to allow the user to evaluate heat pipe designs based on analytic approximations, which are very useful for heat pipe design exploration.

Numerous modeling improvements were implemented in this work to support customer functional requirements and address issues raised by users. This included some major improvements to the capillary pressure model that were designed in collaboration with Argonne National Laboratory. Other modeling improvements improved the accuracy of pressure loss calculations as well as wall heat transfer and wall boiling, which is a prerequisite for modeling the boiling limit of heat pipes.

Due to the increasing interest of users in the near term, training resources have become a high priority, with user examples in particular. Customers identified a number of key examples necessary for their analyses, including demonstrating the ability to provide custom closures, use different types of heat transfer boundary conditions, and capture heat pipe operational limits. In addition to training resources, other documentation requested by users included the details of the spatial discretization.

While Sockeye continues to undergo rapid development, we performed some initial verification and validation to give users a basic demonstration of its capabilities. Comparisons are made between numerical results and some analytic solutions, and results both show excellent agreement and demonstrate some shortcomings of traditional heat pipe analyses. Additionally, we demonstrate theoretical spatial convergence, indicating the correct implementation of the spatial discretization. We performed some initial validation work, and its results indicate good agreement with experimental data but also demonstrate the need for future work. 
Page intentionally left blank 


\section{ACKNOWLEDGEMENTS}

Sockeye development is being carried out under the auspices of Idaho National Laboratory, a contractor of the U.S. Government under contract number DEAC07-05ID14517. Accordingly, the U.S. Government retains a nonexclusive, royalty-free license to publish or reproduce the published form of this contribution, or allows others to do so, for U.S. Government purposes. 
Page intentionally left blank 


\section{CONTENTS}

Summary iii

$\begin{array}{lc}\text { Acknowledgements } & \mathbf{v}\end{array}$

1 Introduction $\quad 1$

2 New Capabilities $\quad 2$

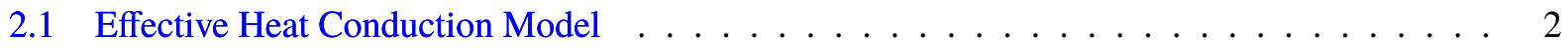

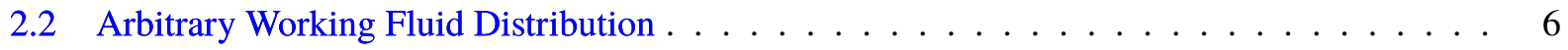

2.3 Variable Permeability . . . . . . . . . . . . . . . . . . . 7

2.4 Variable Meniscus Angle $\ldots \ldots \ldots \ldots \ldots \ldots$

2.5 Design Utilities $\ldots \ldots \ldots \ldots \ldots \ldots \ldots$

2.5 .1 Operational Limits Viewer . . . . . . . . . . . . . . . 11

2.5.2 Frozen Startup Limit Number . . . . . . . . . . . . . . . . . . . . 14

3 Model Improvements $\quad 16$

3.1 Capillary Pressure . . . . . . . . . . . . . . . . . 16

3.1 .1 Excess Liquid Regime $\ldots \ldots \ldots \ldots$. . . . . . . . . . . . . 17

3.1 .2 Saturation Dependence . . . . . . . . . . . . . . . . . 18

3.2 Pressure Losses . . . . . . . . . . . . . . . . . . . . . . . . . . . . . . . . . 19

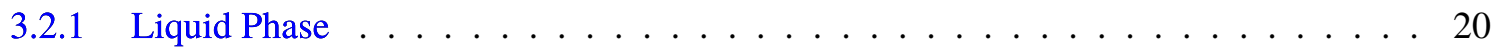

$3.2 .2 \quad$ Vapor Phase . . . . . . . . . . . . . . . . . . . . 22

3.3 Wall Heat Transfer . . . . . . . . . . . . . . . . . . . . . . . . . 22

4 User Documentation and Training 25

4.1 User Examples . . . . . . . . . . . . . . . . . . . . . . . . . 25

4.1 .1 Custom Friction Closures $\ldots \ldots \ldots \ldots \ldots$

4.1.2 Heat Transfer Boundary Conditions . . . . . . . . . . . . . . . . . . 27

4.1 .3 Capillary and Sonic Limits . . . . . . . . . . . . . . . . . . 28

4.2 Discretization Documentation . . . . . . . . . . . . . . . . . 29

5 Verification and Validation $\quad 32$

5.1 Analytic Solutions . . . . . . . . . . . . . . . . . . . . . . . 32

5.2 Spatial Convergence Rate Verification . . . . . . . . . . . . . . . . . . . 39

5.3 SAFE-30 Heat Pipe Module Test . . . . . . . . . . . . . . . . . . . . . 41 
6 Conclusions $\quad 49$

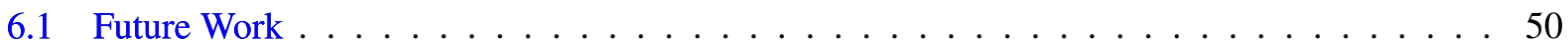

$\begin{array}{ll}\text { References } & 51\end{array}$

\section{FIGURES}

1 Heat pipe cross section and operation of a conventional heat pipe. . . . . . . . . 1

2 Heat pipe cross section with dimensions. . . . . . . . . . . . . . . . . . . . 4

3 Temperature vs. time for the cascading heat pipe failure test problem using the effective heat

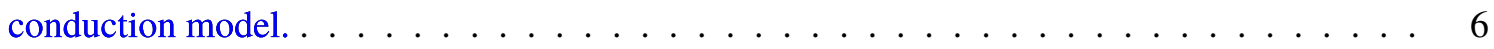

4 Power vs. time for the cascading heat pipe failure test problem using the effective heat conduction model. . . . . . . . . . . . . . . . . . . . 7

5 Effect of variable permeability on the pressure distribution. . . . . . . . . 8

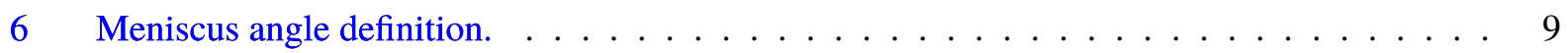

7 Wetting angle of sodium on stainless steel $304 \mathrm{~L} \ldots \ldots \ldots \ldots$

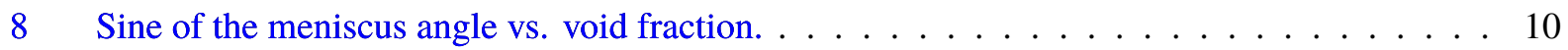

9 Capillary pressure and interfacial area density vs. void fraction. . . . . . . . . . . 10

10 Analytic operating limits utility example. . . . . . . . . . . . . . . . . . . . 12

11 Illustration of liquid-vapor interface vs. void fraction at bounding values. . . . . . . . 17

12 Void fraction and pressure solutions using the old excess liquid capillary pressure relationship. 18

13 Void fraction and pressure solutions using the new excess liquid capillary pressure relationship. 19

14 Capillary pressure example using the $J$-function $J(\zeta)=1-\zeta \ldots \ldots \ldots$

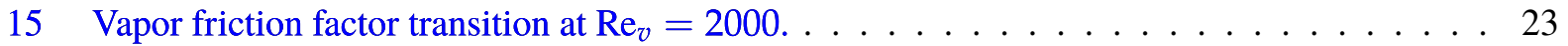

16 Temperature and mass transfers distributions during wall boiling. . . . . . . . . . . . . 25

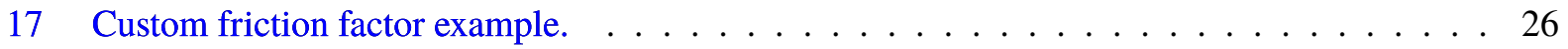

18 Vapor pressure distribution with custom vapor friction factor. . . . . . . . . . . . 27

19 Capillary limit example. . . . . . . . . . . . . . . . . . . . . . . . . 29

20 Sonic limit example. . . . . . . . . . . . . . . . . . . . . 30

$21 x$-t diagrams for the phase $k$ equations. . . . . . . . . . . . . . . 31

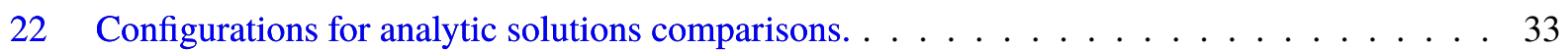

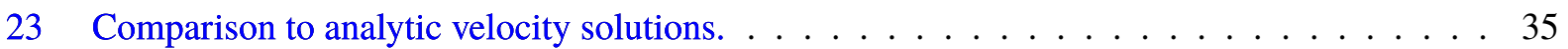

24 Steady temperature solutions for single-ended vs. double-ended configurations. . . . . . . . 36

25 Steady void fraction solutions for single-ended vs. double-ended configurations. . . . . . . . 37

26 Steady pressure solutions for single-ended vs. double-ended configurations. . . . . . . . . 37

27 Comparison of vapor pressure drop with analytic solution for single-ended configuration. . . 38

28 Comparison of liquid pressure drop with analytic solution for single-ended configuration. . . 39 


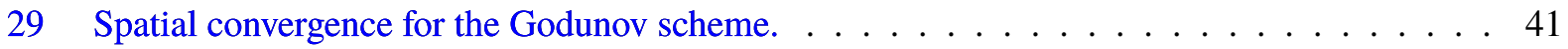

30 Spatial convergence for the full-reconstruction scheme. . . . . . . . . . . . . . . . 42

31 Comparison of experimental and numerical power transients for SAFE-30 test problem. . . . 43

32 Comparison of experimental thermocouple values against temperature solution values at thermocouple positions for SAFE-30 test problem. . . . . . . . . . . . . . 45

33 Final temperature profiles for SAFE-30 test problem. . . . . . . . . . . . . 46

34 Final cladding temperature profile for SAFE-30 test problem . . . . . . . . . . . . 46

35 Final void fraction solutions for SAFE-30 test problem . . . . . . . . . . . . . . 47

36 Final velocity solutions for SAFE-30 test problem. . . . . . . . . . . . . . . . 48

37 Final pressure solutions for SAFE-30 test problem. . . . . . . . . . . . . . . . 49

\section{TABLES}

1 Thermal properties in each region of the effective heat conduction heat pipe model. . . . . 3

2 Comparison of computed FSL values with reference values. . . . . . . . . . . . . 16

3 List of heat transfer examples. . . . . . . . . . . . . . . . . . 28

4 Heat pipe specifications for analytic solutions comparisons. . . . . . . . . . . . . . 34

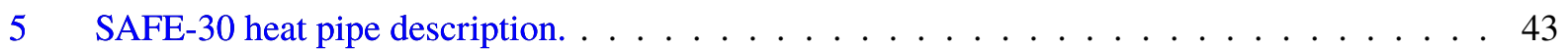

6 SAFE-30 thermocouple locations. . . . . . . . . . . . . . . . . . . . 44 


\section{INTRODUCTION}

Numerous nuclear microreactor designs are currently under development, with an ambitious timeline that includes demonstrations within the next five years. These microreactors are compact enough to be entirely factory-assembled and transported on-demand to locations by truck, railcar, vessel, or airplane, which suits them for many unique applications, such as power generation in remote locations and regions devastated by natural disasters. These designs also boast economic advantages due to the elimination of difficulties associated with large-scale construction projects, a reduced capital cost, and modular scalability. Of these microreactor designs, some of the most promising utilize heat pipe technology to transfer heat from the reactor core to the secondary side heat exchanger, due to the compactness, efficiency, passivity, and reliability of heat pipe technology [1].

Heat pipes are extremely efficient heat transfer devices due to their high effective thermal conductivity, small cross-sectional area, and superior ability to transport heat over great distances. For example, a heat pipe with a 30-mm diameter and $1-\mathrm{m}$ length can transfer $1 \mathrm{~kW}$ of power with a temperature difference of $10^{\circ} \mathrm{C}$, whereas a solid copper rod of the same dimensions would require a temperature difference of $900{ }^{\circ} \mathrm{C}$ [2]. Additionally, heat pipes have a high reliability due to their simple construction, featuring no moving parts. Heat pipe technology has been used in various forms for decades in a wide variety of applications, such as refrigeration, air conditioning, deicing, electronics cooling, oil pipelines, and space reactors [2,3]. The designs of greatest interest for microreactor applications are categorized as "conventional" heat pipes; that is, they each consist of a straight tube (hereafter termed the "cladding"), sealed at both ends, containing a wick structure and a working fluid. An illustration of the cross section of such a heat pipe is shown in Figure 1a, and the principle of operation of this heat pipe is illustrated in Figure 1b. The principle of operation of a heat pipe is illustrated in Figure 1b. Heat is applied at the "evaporator" end of the heat pipe and conducts radially inward into a working fluid, which then vaporizes and traverses to the opposite end of the heat pipe, termed the "condenser" end. Here, the working fluid condenses onto a wick structure, which, through capillary action, draws the liquid working fluid back to the evaporator end, where the cycle begins again. The enormous amount of energy that can be stored as latent heat in the working fluid is the key to the superior thermal efficiency of the heat pipe. The wick structure can take a number of forms, but the form deemed of greatest interest for microreactor heat pipes consists of a wrapped mesh screen.

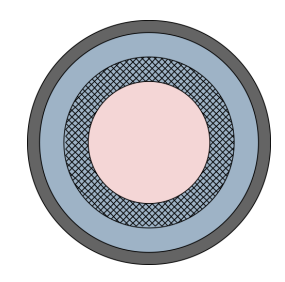

(a) Cross section of a conventional heat pipe. The wick structure is indicated by the cross-hatch pattern.

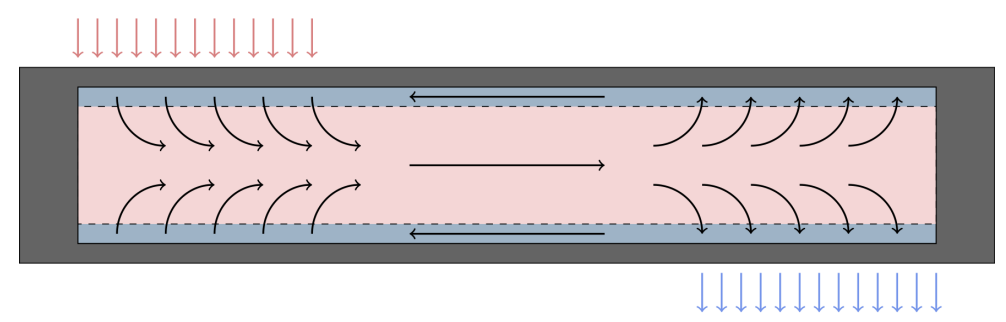

(b) Operation of a conventional heat pipe. Heat input at the evaporator end is shown with red arrows, heat removal at the condenser end is shown with blue arrows, and the working fluid flow path is indicated with black arrows.

Figure 1: Heat pipe cross section and operation of a conventional heat pipe. The vapor phase of the working fluid is shown in pink, the liquid phase of the working fluid is shown in blue, and the cladding is shown in gray.

While reliable, heat pipes are limited by the heat throughput that can be maintained at a given operating temperature. There are a number of different phenomena that lead to these limitations, mostly associated with 
thermal hydraulic aspects of the system. These different limits are the main considerations that need to made in heat pipe design and one of the main complications in heat pipe modeling. Some amount of analysis can be performed analytically but not without serious shortcomings; these analyses employ major assumptions, such as steady conditions and various approximations. While steady conditions may be acceptable for some initial design work, it is important to predict a number of transient phenomena, such as how a heat pipe responds to an operational limit and if and how fast it is able to recover.

To support this effort, the Nuclear Energy Advanced Modeling and Simulation (NEAMS) program began development of Sockeye, an engineering-scale heat pipe tool to be used for the design and safety analysis of nuclear microreactors [4]. Sockeye is built on the Multiphysics Object-Oriented Simulation Environment (MOOSE) [5] framework, which allows for seamless multiphysics coupling with other NEAMS tools, such as BISON and Griffin for thermomechanics and neutronics coupling, respectively. This is demonstrated in the multiphysics application DireWolf, whose objective is the simulation of nuclear microreactors [6].

Sockeye's core capability lies in a 1D, two-phase, compressible flow model, used to simulate the working fluid inside the heat pipe. The engineering-scale design, and thus 1D discretization, is motivated by the computational considerations of a full-scale microreactor assembly, which can include hundreds or thousands of heat pipes; a multidimensional simulation of the flow in all of these heat pipes would be computationally intractable. Literature reviews on the subject of heat pipe modeling reveal a variety of approaches of varying fidelity [2, 7-12]. However, even the most high fidelity of these approaches use simplifying assumptions, such as that of a perfectly saturated wick. Also, none of the existing simulation tools present a demonstration of operational limits, instead restricting their scope to stay within safe operational limits. Capturing and modeling the transient effects of these limits is a key driver of Sockeye's models.

Development of this tool is largely guided by the needs of key stakeholders, such as Westinghouse Electric Company, currently developing the eVinci ${ }^{\mathrm{TM}}$ Micro Reactor [13]. Regular meetings with Westinghouse were used to discuss their functional requirements for heat pipe modeling as well as provide an opportunity for user feedback. This report describes a number of improvements that support these needs and is organized as follows. Section 2 discusses new capabilities, Section 3 discusses model improvements, Section 4 discusses documentation and user examples, Section 5 discusses verification and validation, and Section 6 discusses some conclusions of this work. Note that unless otherwise specified, the results shown in this report were generated using the following version of Sockeye: pub/inl-ext-21-61673.

\section{NEW CAPABILITIES}

This section discusses a number of new Sockeye capabilities. Section 2.1 describes an alternative heat pipe model that offers a simple, robust, and complementary surrogate for Sockeye's flow model. Sections 2.2, 2.3, and 2.4 describe user flexibility improvements, namely the ability to specify an arbitrary working fluid distribution, the ability to vary wick permeability in space and time, and the ability to specify the range of curvature of the liquid-vapor interface, respectively. Section 2.5 describes user utilities that provide the ability to explore heat pipe design considerations before simulation.

\subsection{Effective Heat Conduction Model}

After the startup phase of a heat pipe, a heat pipe can be idealized as a solid cylinder with a very high thermal conductivity, thus ignoring its internal mechanisms, which can be difficult to model. Many researchers have used this analogy to model heat pipes because of its relative simplicity and its ability to accurately capture thermal response in many cases $[14,15]$. The idea is simply to solve the heat conduction 
equation in $2 \mathrm{D}$ or $3 \mathrm{D}$ and use thermal properties that yield a rod of superconductivity.

In the context of Sockeye, this model represents an alternative to the existing heat pipe model, which solves 1D flow equations in the interior of the heat pipe and couples them to $2 \mathrm{D}$ heat conduction equations solved in the cladding alone. The effective heat conduction model offers a number of advantages over the flow model. The training burden for this model is much lower, as there is a single solution variable, temperature, that users need to understand and process. The model is much simpler, being composed of the heat conduction equation alone, and thus its implementation and maintenance require less time and effort. Finally, its usage is more robust in terms of run failure; convergence is easier to achieve, since it does distinguish many of the regimes subject to difficulties for the flow model. However, the model does have serious fidelity shortcomings, compared to the flow model. It does not model the operational limits mechanistically and thus relies on coarse approximations to the limits, which will be described later in this section. Sockeye's implementation of both models allows for a complementary modeling approach, where the model can be chosen based on the needs of a particular problem.

The heat conduction equation is expressed as follows:

$$
\rho c_{p} \frac{\partial T}{\partial t}-\nabla \cdot(k \nabla T)=0
$$

where $\rho$ is density, $c_{p}$ is specific heat capacity, $k$ is thermal conductivity, and $T$ is temperature. To take the effective heat conduction approach, one can either use thermal properties that are averaged over the entire cross section of the heat pipe, or one can use different thermal properties in different radial regions. Sockeye takes the latter approach, as it offers a more accurate temperature distribution. Table 1 summarizes the different regions and different thermal properties used in each region.

Table 1: Thermal properties in each region of the effective heat conduction heat pipe model.

\begin{tabular}{lccc}
\hline Radial Region & $\rho$ & $c_{p}$ & $k$ \\
\hline Cladding & $\rho_{\text {clad }}$ & $c_{p, \text { clad }}$ & $k_{\text {clad }}$ \\
Wick/Annulus & $\rho_{\text {wick/ann }}$ & $c_{p \text {,wick/ann }}$ & $k_{\text {wick/ann }}$ \\
Core & $\rho_{v}$ & $c_{p, v}$ & $k_{\text {core }}$ \\
\hline
\end{tabular}

In the cladding region, the thermal properties are simply equal to the thermal properties of the cladding material, just like in the flow model. The wick/annulus region includes the liquid-saturated wick structure and the liquid annulus, if any. Its thermal properties are simply area averages of the constituent wick matrix and liquid phase thermal properties:

$$
\begin{gathered}
\rho_{\text {wick } / \mathrm{ann}}=\frac{\epsilon A_{\text {wick }} \rho_{\ell}+(1-\epsilon) A_{\text {wick }} \rho_{\text {wick }}+A_{\text {ann }} \rho_{\ell}}{A_{\text {wick }}+A_{\text {ann }}}, \\
c_{p \text {,wick/ann }}=\frac{\epsilon A_{\text {wick }} c_{p, \ell}+(1-\epsilon) A_{\text {wick }} c_{p \text {,wick }}+A_{\text {ann }} c_{p, \ell}}{A_{\text {wick }}+A_{\text {ann }}}, \\
k_{\text {wick/ann }}=\frac{\epsilon A_{\text {wick }} k_{\ell}+(1-\epsilon) A_{\text {wick }} k_{\text {wick }}+A_{\text {ann }} k_{\ell}}{A_{\text {wick }}+A_{\text {ann }}},
\end{gathered}
$$

where $\epsilon$ is the porosity of the wick and the cross-sectional areas of the wick and annulus regions are defined as follows:

$$
A_{\text {wick }}=\frac{\pi}{4}\left(D_{\text {wick }, \mathrm{o}}^{2}-D_{\text {wick }, \mathrm{i}}^{2}\right),
$$




$$
A_{\text {ann }}=\frac{\pi}{4}\left(D_{\text {clad,i }}^{2}-D_{\text {wick,o }}^{2}\right),
$$

with the diameters illustrated in Figure 2. In the vapor core region, the density and specific heat capacity are taken to be those of the vapor phase of the working fluid. The thermal conductivity, however, is chosen to reflect the effective heat conductance of heat pipe operation. Typically, an arbitrary, high value is used, such as $k_{\text {core }}=10^{5} \mathrm{~W} / \mathrm{m}$.

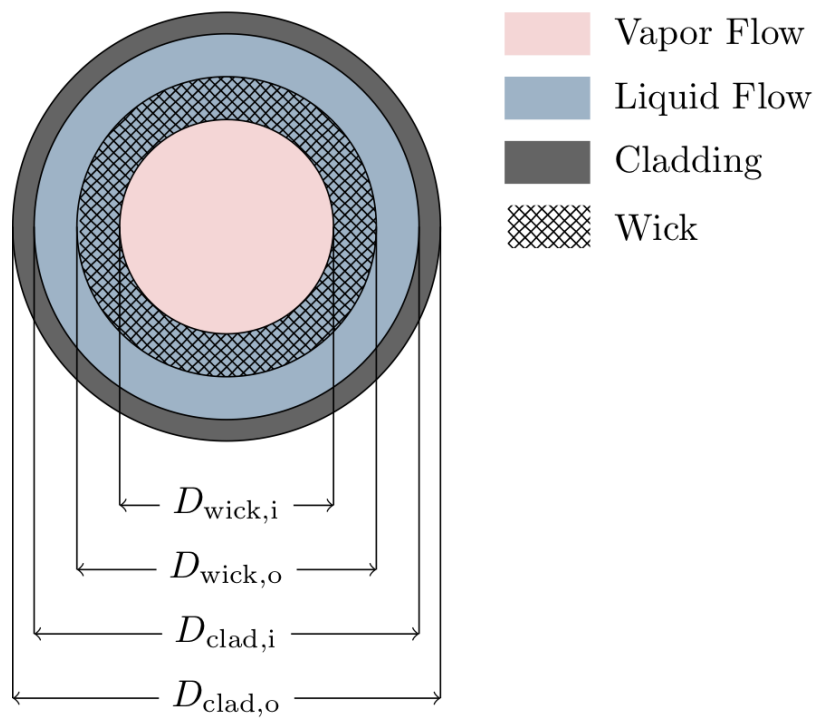

Figure 2: Heat pipe cross section with dimensions.

Now we discuss the approach of modeling operational limits. Recall that heat pipe operational limits are effectively upper bounds for the heat throughput. In this effective heat conduction model, we enforce these upper bounds at the boundary conditions on either the evaporator or condenser end of the heat pipe. In reality, the heat bottleneck location varies by the operational limit. In the sonic limit, for example, flow is choked at the evaporator exit, so heat transport should be limited at that location instead. However, we adopted the approach of enforcing these limits at the boundary conditions due to its simplicity and generality.

The enforcement of the operational limits at the boundary conditions is performed by applying a scaling factor $\gamma$ to the boundary conditions of the chosen end of the heat pipe. That is, the nominal (i.e., before scaling) heat flux function $q$ is replaced by $\gamma q$. The nominal heat rate $\dot{Q}$ is computed as follows:

$$
\dot{Q}=\int_{\Gamma} q \mathrm{~d} \Gamma
$$

where $\Gamma$ is the relevant boundary of the problem domain. The scaling factor is separated into two components: $\gamma=\gamma_{\text {rec }} \gamma_{\text {cat }}$, where $\gamma_{\text {rec }}$ corresponds to those limits that are considered "recoverable" and $\gamma_{\text {cat }}$ corresponds to those limits that are considered "catastrophic." Here, "recoverable" means that the limit is temporary; the limit is naturally resolved as temperature increases. This is the case for the viscous and sonic limits; see, for example, Figure 10. The other limits lead to a dry-out condition, and thus intervention is required to recover the operation of the heat pipe; these are referred as "catastrophic" limits here.

To compute the scaling factors, first the minimum limit in each category is computed:

$$
\dot{Q}_{\mathrm{rec}}=\min _{j} \dot{Q}_{j}^{\mathrm{rec}},
$$




$$
\dot{Q}_{\text {cat }}=\min _{j} \dot{Q}_{j}^{\text {cat }},
$$

where the individual heat rates $\dot{Q}_{j}^{\text {rec }}$ and $\dot{Q}_{j}^{\text {cat }}$ are estimated using the analytic approximations discussed in Section 2.5.1. Recall that these expressions are functions of temperature. The temperature used in these evaluations is the average temperature in the vapor core region $\Omega_{\text {core: }}$ :

$$
\bar{T}_{\text {core }}=\frac{1}{V_{\text {core }}} \int_{\Omega_{\text {core }}} T \mathrm{~d} V,
$$

where $V_{\text {core }}$ is the volume of the core region. The recoverable scaling factor is determined as follows:

$$
\gamma_{\mathrm{rec}}=\left\{\begin{array}{ll}
1 & \dot{Q} \leq \dot{Q}_{\mathrm{rec}} \\
\frac{\dot{Q}_{\mathrm{rec}}}{\dot{Q}} & \dot{Q}>\dot{Q}_{\mathrm{rec}}
\end{array} .\right.
$$

The catastrophic scaling factor is simply a switch for the boundary conditions:

$$
\gamma_{\text {cat }}=\left\{\begin{array}{cc}
1 & \dot{Q} \leq \dot{Q}_{\text {cat }} \\
0 & \dot{Q}>\dot{Q}_{\text {cat }}
\end{array} .\right.
$$

Once the catastrophic scaling factor is set to zero, it remains zero for the remainder of the transient.

Now we give a demonstration of the capability of this model. The test problem shows how a cascading heat pipe failure might be modeled. Consider a heat pipe in a large hexagonal core block assembly, such that it has six neighboring heat pipes. Initially, each heat pipe is removing heat at the rate of $\dot{Q}_{\text {single. If a }}$ neighboring heat pipe fails, its heat removal responsibility is assumed to fall equally to its six neighbors, one of which includes the original heat pipe in question, thereby increasing its power by $\dot{Q}_{\text {single }} / 6$. This test problem models the transient associated with multiple heat pipe failures and demonstrates how a heat pipe might be able to sustain the heat rate from a number of adjacent heat pipe failures. The chosen heat rate applied to the boundary is as follows:

$$
\dot{Q}(t)= \begin{cases}\dot{Q}_{\text {single }} & t<200 \mathrm{~s} \\ \dot{Q}_{\text {single }}+\frac{1}{6} \dot{Q}_{\text {single }} & 200 \mathrm{~s} \leq t<400 \mathrm{~s} \\ \dot{Q}_{\text {single }}+\frac{2}{6} \dot{Q}_{\text {single }} & t \geq 400 \mathrm{~s}\end{cases}
$$

Figure 3 shows the resulting transient of the temperature for this problem. At $200 \mathrm{~s}$, the temperature had nearly approached a steady value, but, at this time, a neighboring heat pipe fails, causing the temperature to seek a new steady value. This occurs again with another failure at $400 \mathrm{~s}$, but, with this failure, the modeled heat pipe eventually fails during the climb to a new steady value, causing its temperature to climb rapidly due to the lack of heat removal from the heat pipe. In this problem, the scaling factor $\gamma$ was applied to the condenser boundary; thus heat is not rejected from entering the heat pipe but from leaving it.

Figure 4 gives more context for the events by showing power vs. time. Each of the operational limits are shown, as well as the nominal (before limitation) power at the condenser, $\dot{Q}_{\text {cond }}$, labeled as "Condenser Power (Unlimited)," and the scaled power at the condenser, $\gamma \dot{Q}_{\text {cond }}$, labeled as "Condenser Power." As mentioned previously, the sonic and viscous limits are considered recoverable and thus contribute to $\gamma_{\text {rec }}$, while the others contribute to $\gamma_{\text {cat }}$. At the beginning of the transient, the temperature is relatively low, where the sonic and viscous limits are restrictive; thus, the condenser power is limited and follows the minimum of these two limits. At around $50 \mathrm{~s}$, the heat pipe is no longer viscous-limited or sonic-limited and approaches the $\dot{Q}_{\text {single }}$ value, until $200 \mathrm{~s}$, when $\dot{Q}_{\text {single }} / 6$ is added and then again at $400 \mathrm{~s}$, when another $\dot{Q}_{\text {single }} / 6$ is added. After this second adjacent heat pipe failure, the power removed at the condenser eventually matches the capillary 


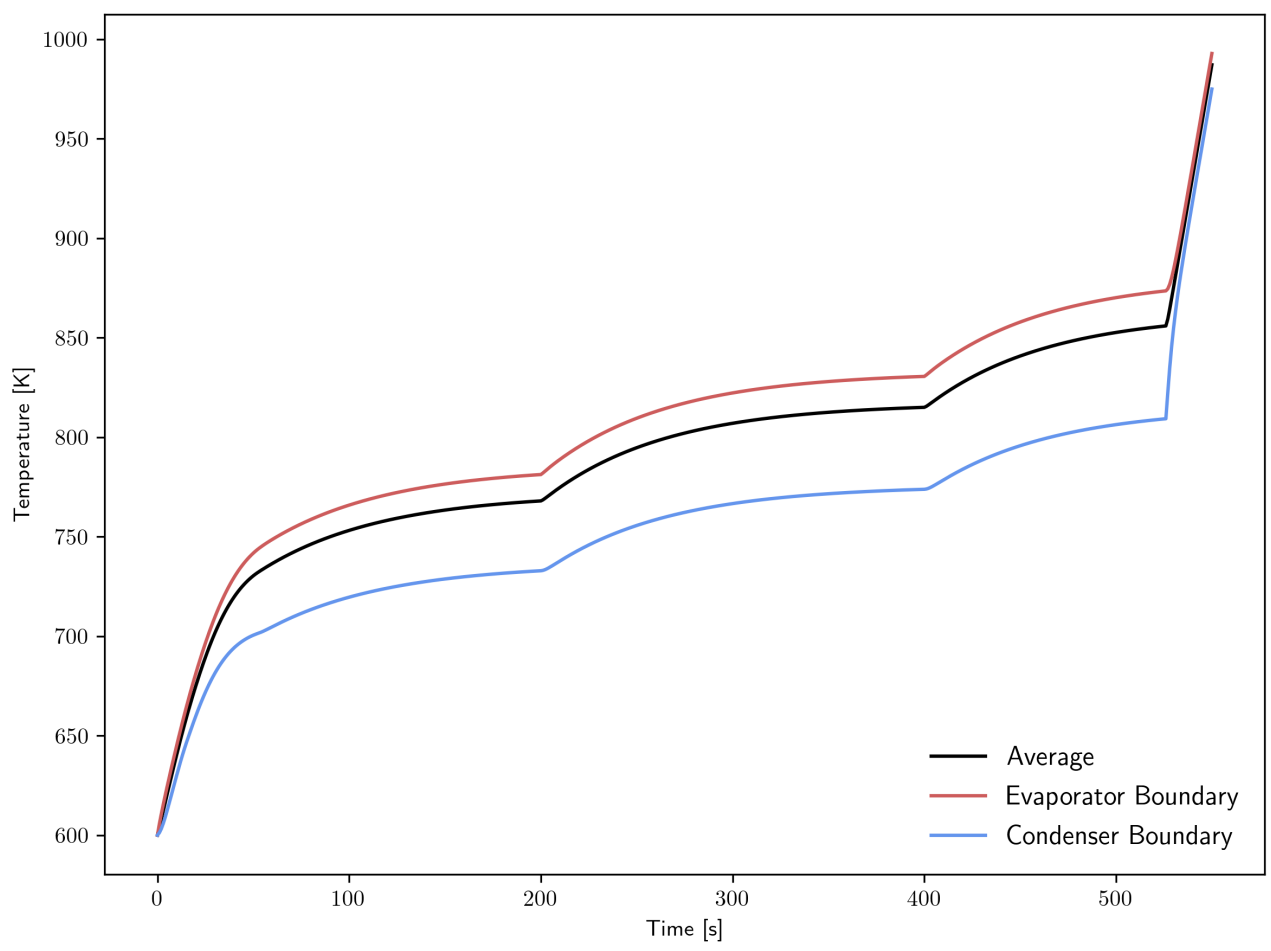

Figure 3: Temperature vs. time for the cascading heat pipe failure test problem using the effective heat conduction model.

limit, causing the failure of the modeled heat pipe. Power removed is forced to zero with $\gamma_{\text {cat }}=0$, causing a runaway temperature excursion, thus demonstrating a cascading heat pipe failure.

The model described in this section is simple, and there are numerous improvements that can be made. Firstly, each limit can be treated independently with custom behavior. The limits restrict heat flow at different locations and have different effects. For example, the capillary limit eventually leads to a dry-out condition. To model this more accurately, this dryout can be approximated by adjusting thermal properties in the wickannulus region to reflect those of vapor instead of liquid. Another improvement that can be made would be to account for the latent heat of fusion during melting. This energy is not insignificant and can thus significantly shift the timing of temperature rises and declines during startup and shutdown, respectively. However, it should be noted that this accounting requires the use of a different formulation of heat conduction, using internal energy as the solution variable instead of temperature. Furthermore, this would also require a problem division between the cladding region and other regions, since the cladding region would still use the original heat conduction equations. This approach may introduce numerical difficulties. Other improvements that can be made to the model include various predictions of lengths of regions, like pools of excess working fluid and noncondensable gases, which can be leveraged for a more accurate prediction of heat transfer at the secondary side.

\subsection{Arbitrary Working Fluid Distribution}

The total working fluid inventory in a heat pipe is an important design factor that other tools implicitly do not consider since they assume a perfectly saturated wick. The working fluid inventory affects not only 


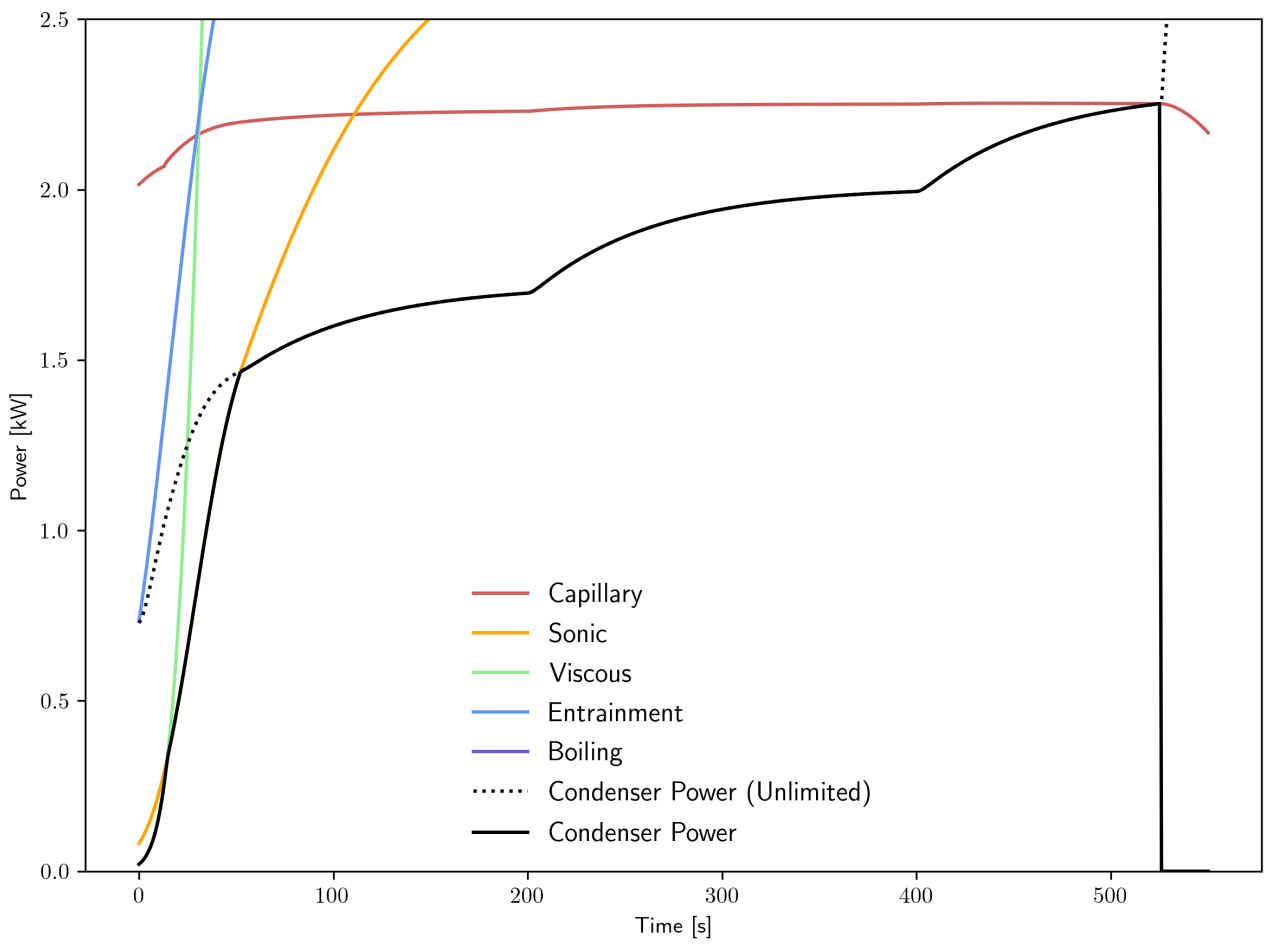

Figure 4: Power vs. time for the cascading heat pipe failure test problem using the effective heat conduction model.

the total heat capacity of the heat pipe but also plays an important role with respect to operational limits. The initial distribution of the working fluid varies for frozen startup and, during normal operation, generally does not attain uniformity, often due to the presence of a condenser pool of excess working fluid.

\subsection{Variable Permeability}

Heat pipes in microreactors are operated at high temperatures, which increases the potential for solubility corrosion and mass transfer, which can degrade the capillary action of the wick structure. Soluble material can be transported over time by the liquid phase from the condenser region and precipitate on the wick in the evaporator region as the working fluid evaporates [16]. While the chemical processes are complicated, it is possible to approximate their effects by modifying wick properties spatially and temporally.

The permeability can now be input by the user as a function of space and time as a first step toward variable wick properties. The variable porosity is more complex to implement as it makes the wick volume fraction variable as well and, thus, has impact on the conservation equations.

Figure 5a shows an example comparing the pressure distributions for a case where the permeability varies over the length of the heat pipe vs. the same heat pipe with a uniform permeability. The permeability distributions are shown in Figure 5b. The uniform permeability case uses the average of the permeability in the variable case. The evaporator is $0.5 \mathrm{~m}$ long, then the adiabatic region goes from $0.5 \mathrm{~m}$ to $1.5 \mathrm{~m}$, and the final $0.5 \mathrm{~m}$ corresponds to the condenser region. In the condenser region, the variable permeability is higher than the uniform permeability, and Figure 5a shows that the pressure drop in that region is smaller with the 
variable permeability. As the permeability continues to drop, the pressure drop increases and is much higher in the evaporator region. In this example, the variable permeability could adversely impact the performance of the heat pipe since its higher pressure drop poses a greater risk for the capillary limit.

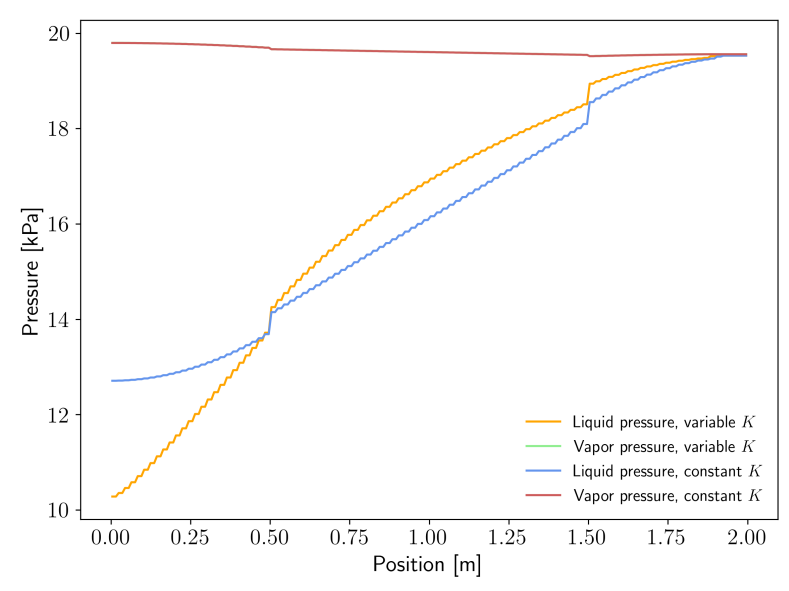

(a) Pressure distributions

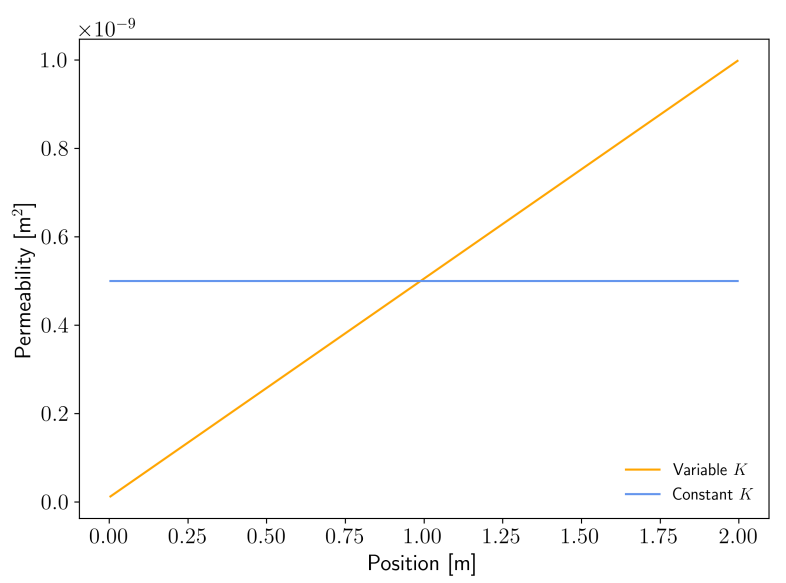

(b) Permeability

Figure 5: Effect of variable permeability on the pressure distribution.

\subsection{Variable Meniscus Angle}

The capillary pressure difference between the phases and the interfacial area density both depend on the curvature of the liquid-vapor interface. We assumed that the interface has a spherical cap shape with a radius of $R_{\text {men }}$. The meniscus angle of this interface, $\theta_{\text {men }}$, is defined as

$$
\cos \theta_{\text {men }}=\frac{R_{\text {pore }}}{R_{\text {men }}}
$$

where $R_{\text {men }}$ is the curvature radius of the interface. Figure 6 gives an illustration of the meniscus angle. Note that this picture is not a physical illustration of the liquid-vapor-solid contact angles but a demonstration of how the meniscus angle is determined in Sockeye.

The details of the calculation of the meniscus angle, depending on the location of the liquid-vapor interface, are given in the theory manual [17]. Sockeye's default closures assume that the curvature of the liquid-vapor interface can range from being flat to being fully hemispherical in each pore. It is now possible to restrict the maximum curvature by specifying the closure to compute the minimum contact angle, using a Material. One major use of this closure is to specify the temperature dependence of the minimum contact angle. Another use might be to capture the degradation of the wetting ability of the wick due to contamination or other factors.

Figure 7 shows that the contact angle of sodium on stainless steel $304 \mathrm{~L}$ has a strong temperature dependence. Below $400{ }^{\circ} \mathrm{C}$, the liquid phase is not wetting the solid. Above $400{ }^{\circ} \mathrm{C}$, the wetting angle decreases, and almost perfect wetting occurs above $570{ }^{\circ} \mathrm{C}$. Thus, before the liquid wets the solid with a small contact angle, the pumping action of the wick will be very limited. This will strongly impact the performance of the heat pipe and thus needs to be included in the heat pipe model. 


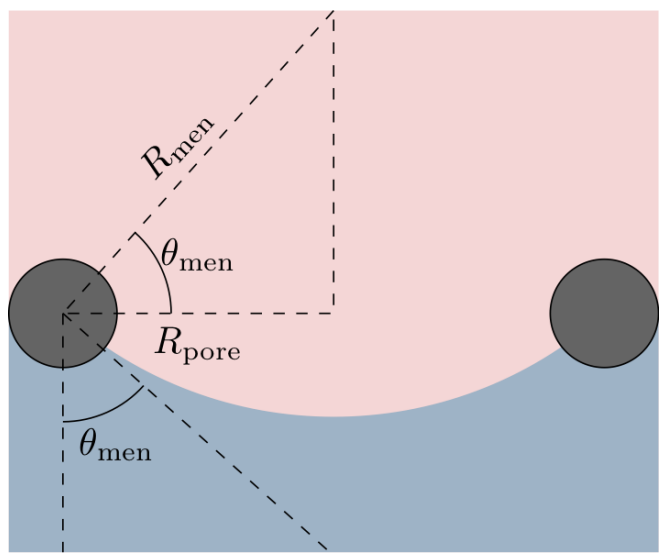

Figure 6: Meniscus angle definition.

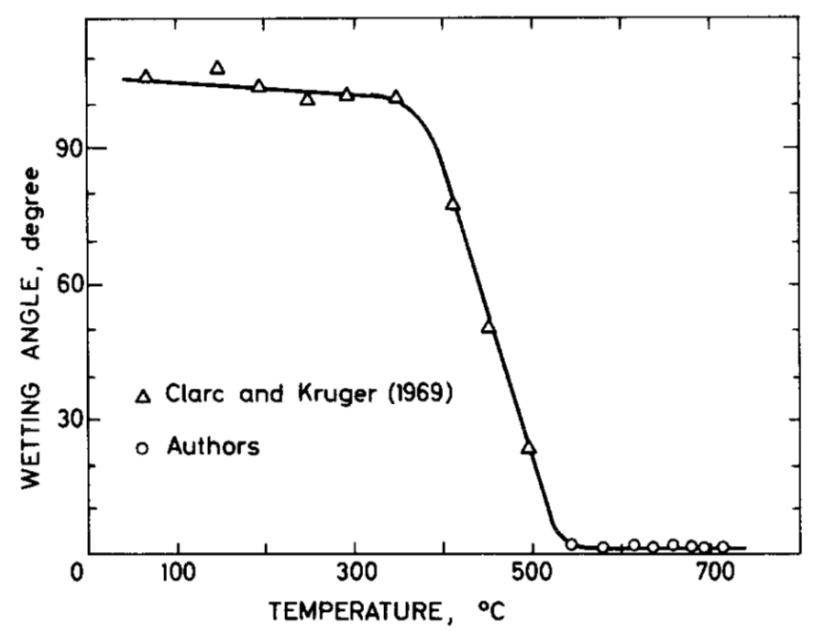

Figure 7: Wetting angle of sodium on stainless steel 304 L [18].

Figure 8 shows an example of the sine of the meniscus angle as a function of the void fraction for the perfect wetting case and for the meniscus angle limited to $30^{\circ}$. When the liquid-vapor interface is located at the inner wick surface, it is assumed to be flat. As the void fraction increases, the curvature of the interface increases to reach the maximum curvature defined by the minimum meniscus angle. Past this point, the interface recesses in the wick structure.

The effect of limiting the meniscus angle on the capillary pressure and on the interfacial area density is shown in Figure 9a and in Figure 9b. 


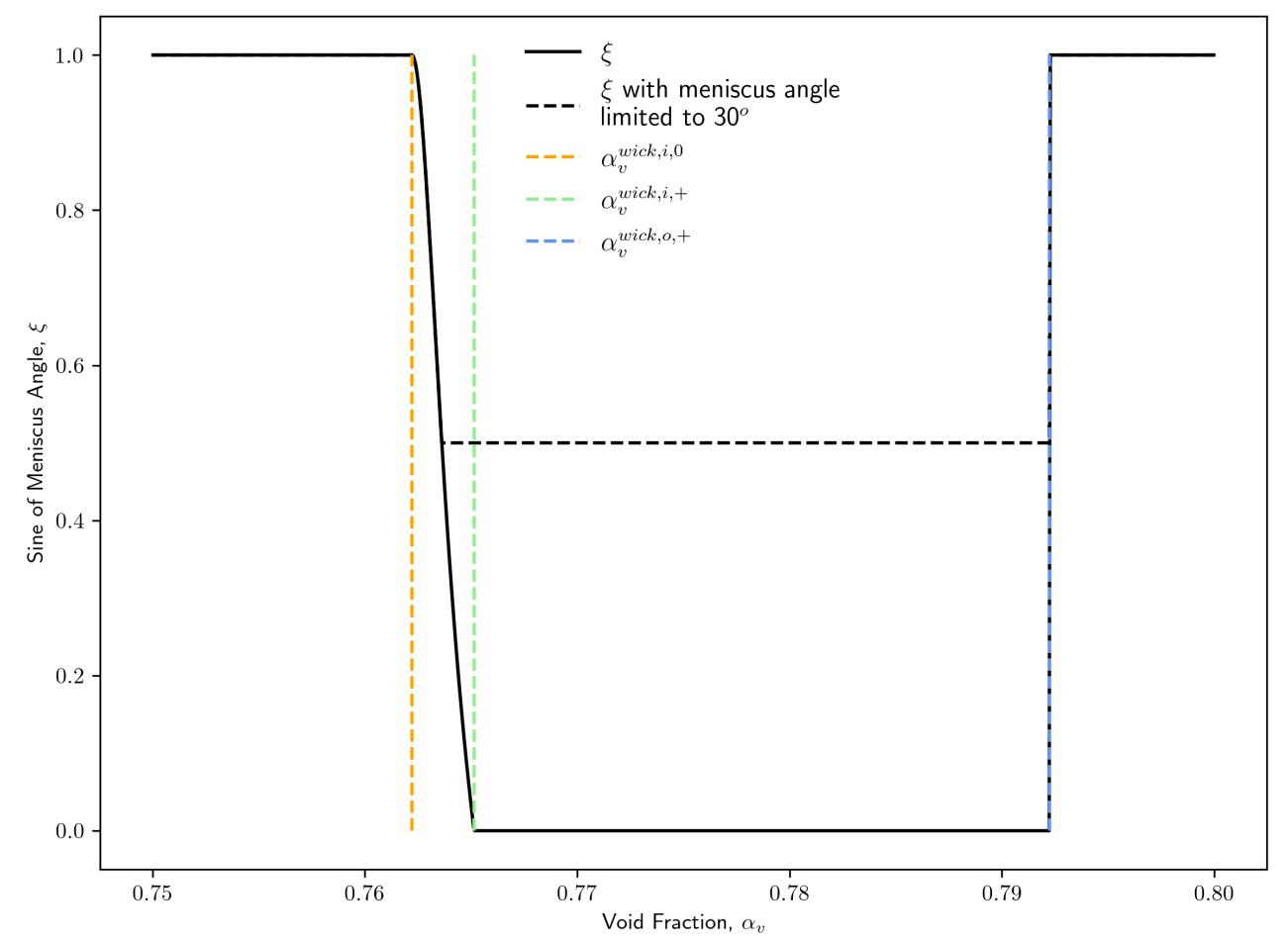

Figure 8: Sine of the meniscus angle vs. void fraction.

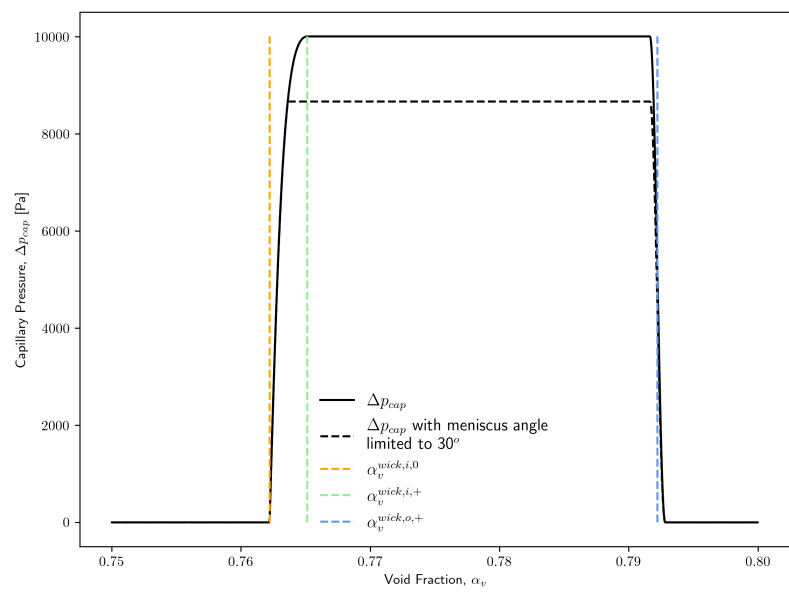

(a) Capillary pressure.

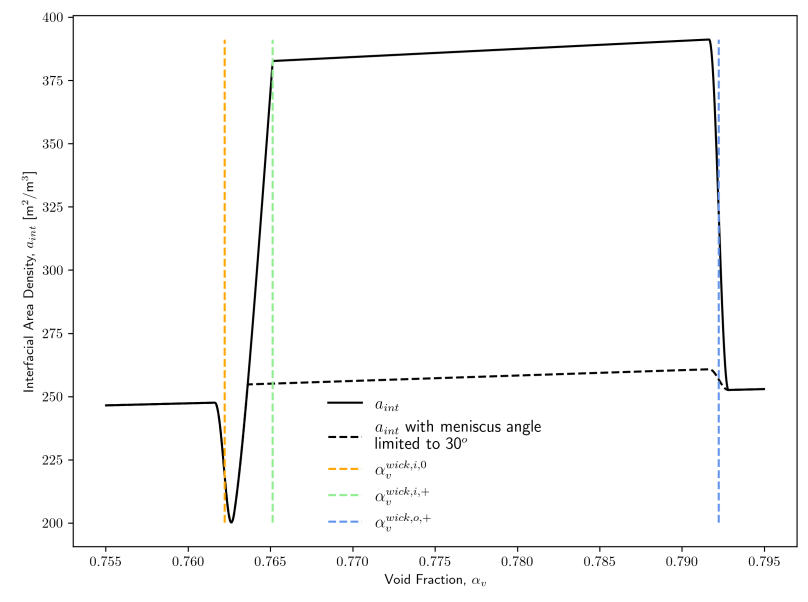

(b) Interfacial area density.

Figure 9: Capillary pressure and interfacial area density vs. void fraction. 


\subsection{Design Utilities}

\subsubsection{Operational Limits Viewer}

Heat pipes are very reliable heat transfer devices, but they are limited in their power throughput by a number of phenomena. The most commonly cited limits are the following [2]:

- The capillary limit

- The entrainment limit

- The sonic limit

- The viscous limit

- The boiling limit.

Descriptions of these limits are given later in this section. These limits are a primary consideration in heat pipe design, and, though Sockeye aims to reproduce each of these limits through simulation, it is advantageous to include analytic approximations to these limits as a first step in heat pipe analysis. The recommended workflow is to design a heat pipe based on an approximation of heat pipe limits and then perform a simulation to assess the heat pipe's performance in a variety of scenarios. Also, these analytic approximations can be used as a means of verification of Sockeye's modeling of these limits. Such examples are given in Section 4.1.3 for the viscous and sonic limits.

The user interface for using these analytic approximations is designed to share a similar structure to that of a simulation's input file, which allows for a more seamless transition between design and simulation steps. The approximations often make assumptions regarding the heat pipe heating configuration (see a discussion in Section 5.1), so unfortunately the design utility described here does not have the full flexibility of Sockeye's flow model, and thus there is no clear path for an automatic design assessment from simulation input file. Nevertheless, this utility provides a very useful capability for a vast majority of cases. After running a design input file with the Sockeye executable, the user simply uses a provided plot script to produce a plot of the operating envelope. An example is shown in Figure 10.

Now we briefly discuss the analytic approximations of each of the operating limits. For more detail, please see the theory manual [17] and [2].

Capillary Limit The capillary limit is derived by comparing the sum of the pressure drops around the heat pipe to the maximum pressure difference $\Delta p_{\text {cap,max }}$ that can be supported at the liquid-vapor interface:

$$
\Delta p_{\text {evap }}+\Delta p_{v}+\Delta p_{\text {cond }}+\Delta p_{\ell, \text { flow }}+\Delta p_{\ell, g} \leq \Delta p_{\text {cap, } \max },
$$

where

- $\Delta p_{\text {cap,max }}$ is the maximum pressure difference that can be supported at the liquid-vapor interface

- $\Delta p_{\text {evap }}$ is the pressure drop due to evaporation

- $\Delta p_{\text {cond }}$ is the pressure drop due to condensation

- $\Delta p_{v}$ is the pressure drop on the vapor side due to acceleration, friction, etc. 


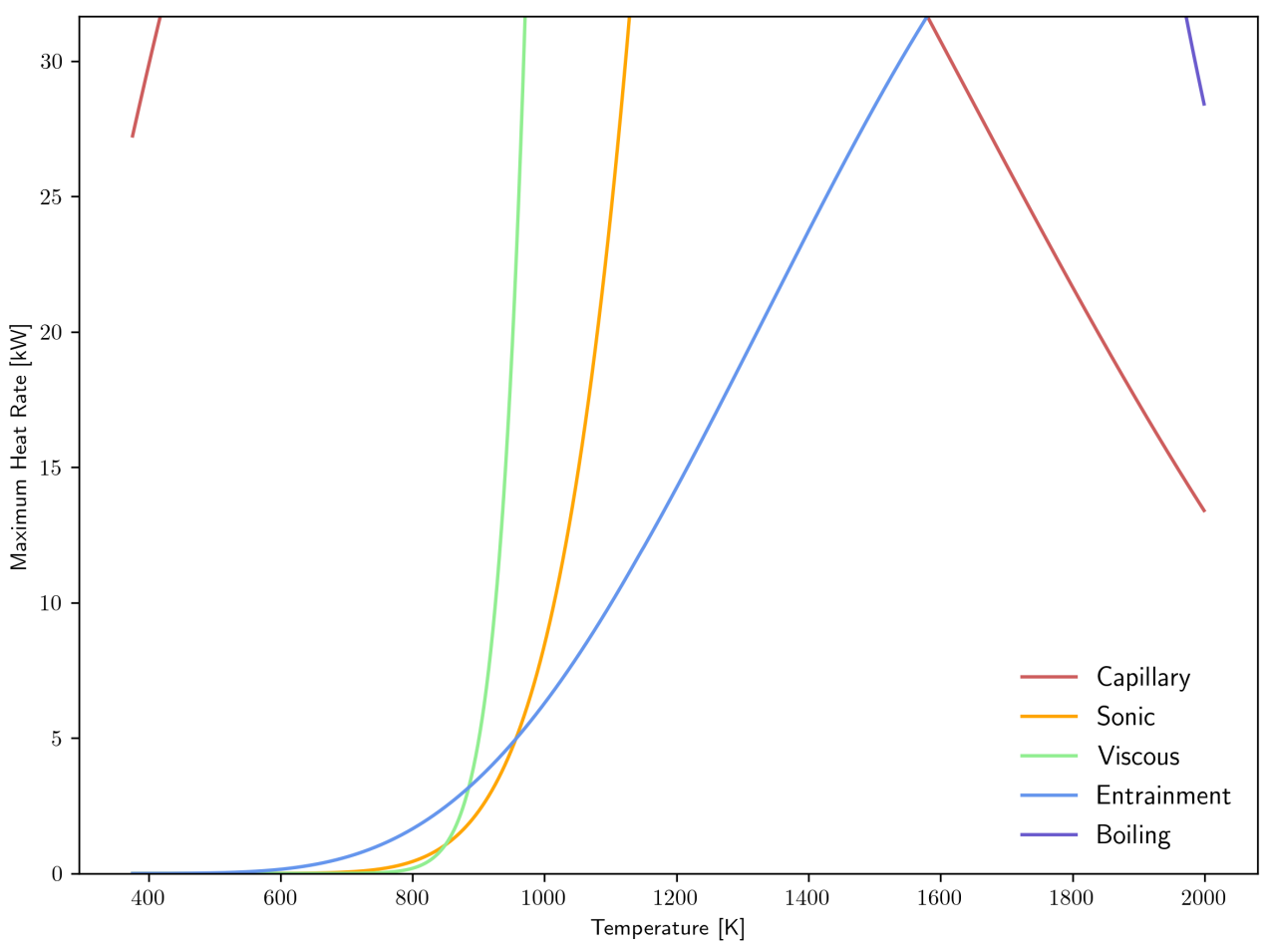

Figure 10: Analytic operating limits utility example.

- $\Delta p_{\ell, \text { flow }}$ is the pressure drop on the liquid side due to friction and porous drag

- $\Delta p_{\ell, g}$ is the pressure drop on the liquid side due to gravity (note this is negligible for the vapor side).

The maximum capillary pressure is given by

$$
\Delta p_{\text {cap }, \max }=\frac{2 \sigma}{R_{\mathrm{eff}}}
$$

where $R_{\text {eff }}$ is the effective pore radius, which is ideally determined experimentally, but can be estimated analytically if needed.

Neglecting $\Delta p_{\text {evap }}, \Delta p_{\text {cond }}$, and $\Delta p_{v}$ gives

$$
\Delta p_{\ell, \text { flow }}+\Delta p_{\ell, g} \leq \Delta p_{\text {cap }, \max } .
$$

The gravity pressure drop is computed as

$$
\Delta p_{\ell, g}=\rho_{\ell} g L_{\mathrm{hp}} \sin \theta,
$$

where

- $L_{\mathrm{hp}}$ is the total length of the heat pipe

- $\theta$ is the angle between the heat pipe and the horizontal at the condenser end (thus, $\theta=0$ corresponds to horizontal, $\theta=-\frac{\pi}{2}$ corresponds to gravity assisted, and $\theta=\frac{\pi}{2}$ corresponds to gravity adverse). 
Using Darcy's law, the liquid flow pressure drop is as follows:

$$
\Delta p_{\ell, \text { flow }}=\frac{\mu_{\ell} L_{\mathrm{eff}} \dot{m}}{\rho_{\ell} K A_{\ell}}
$$

where

- $L_{\mathrm{eff}}=\frac{1}{2} L_{\mathrm{evap}}+L_{\mathrm{adia}}+\frac{1}{2} L_{\mathrm{cond}}$ is the effective length

- $\dot{m}$ is the mass flow rate

- $K$ is the permeability of the liquid channel

- $A_{\ell}$ is the cross-sectional area of the liquid channel.

Using $\dot{Q}=\dot{m} h_{\text {lat }}$ (where $h_{\text {lat }}$ is the latent heat), the capillary limit is obtained [2, 3]:

$$
\begin{gathered}
\dot{Q} \leq \dot{Q}_{\text {max,cap }} \\
\dot{Q}_{\text {max }, \text { cap }}=\frac{\rho_{\ell} \sigma h_{\text {lat }}}{\mu_{\ell}} \frac{K A_{\ell}}{L_{\mathrm{eff}}}\left(\frac{2}{R_{\mathrm{eff}}}-\frac{\rho_{\ell} g L_{\mathrm{hp}}}{\sigma} \sin \theta\right) .
\end{gathered}
$$

Sonic Limit The sonic limit occurs because the flow becomes choked when it becomes sonic. An estimate for this limit is as follows [2]:

$$
\dot{Q}_{\text {max }, \text { son }}=\frac{\rho_{v} c_{v} h_{\mathrm{lat}} A_{v}}{\left(2\left(\gamma_{v}+1\right)\right)^{1 / 2}}
$$

where

- $\rho_{v}, c_{v}$, and $\gamma_{v}$ are the vapor density, sound speed, and ratio of specific heats $\left(c_{p} / c_{v}\right)$, respectively, all evaluated at the evaporator end cap

- $A_{v}=\frac{\pi D_{v}^{2}}{4}$ is the cross-sectional area of the vapor channel.

Viscous Limit The viscous limit occurs because the vapor pressure at the condenser end cap cannot be less than zero. By performing some analyses in the viscous flow regime, the following expression is obtained for the maximum heat transfer rate due to the viscous limit [2]:

$$
\dot{Q}_{\text {max }, \text { vis }}=\frac{A_{v}^{2} h_{\text {lat }} \rho_{v} p_{v}}{16 \pi \mu_{v} L_{\mathrm{eff}}}
$$

where

- $A_{v}=\frac{\pi D_{v}^{2}}{4}$ is the cross-sectional area of the vapor channel

- $\rho_{v}$ and $p_{v}$ correspond to the vapor density and pressure, respectively, at the evaporator end cap

- $L_{\text {eff }}=\frac{1}{2} L_{\text {evap }}+L_{\text {adia }}+\frac{1}{2} L_{\text {cond }}$ is the effective length. 
Entrainment Limit The entrainment limit exists because the vapor phase can shear off liquid into the core vapor channel, thus reducing the rate at which liquid can return to the evaporator. Entrainment occurs when the shear force of the vapor overcomes the surface tension force. The Weber number is a dimensionless number that is a ratio of these two forces, and entrainment occurs when this number is greater than unity, thus leading to the following heat transfer rate limit [2]:

$$
\dot{Q}_{\text {max ent }}=A_{v} h_{\text {lat }}\left(\frac{\sigma \rho_{v}}{D_{h, \text { pore }}}\right)^{1 / 2},
$$

where

- $A_{v}=\frac{\pi D_{v}^{2}}{4}$ is the cross-sectional area of the vapor channel

- $D_{h \text {,pore }}$ is the hydraulic diameter of a pore.

Boiling Limit The boiling limit occurs because bubble formation and departure can inhibit the ability of the liquid to return to the evaporator. This limit is estimated as follows [2]:

$$
\dot{Q}_{\text {max boil }}=\frac{4 \pi L_{\mathrm{evap}} k_{\mathrm{eff}} \sigma T_{v}}{h_{\mathrm{lat}} \rho_{v} \ln \left(D_{\mathrm{hp}, \mathrm{i}} / D_{v}\right)}\left(\frac{1}{R_{b}}-\frac{1}{R_{\mathrm{men}}}\right)
$$

where

- $k_{\text {eff }}$ is the effective thermal conductivity of the wick, which in general depends on the geometry and thermal conductivity of the wick structure itself, as well as the thermal conductivity of the liquid

- $D_{\mathrm{hp}, \mathrm{i}}$ is the inner heat pipe wall diameter

- $D_{v}$ is the vapor channel diameter

- $R_{b}$ is the bubble radius ([2] notes that this could be taken to be $10^{-7} \mathrm{~m}$ for conventional heat pipes)

- $R_{\text {men }}$ is the meniscus radius at the liquid-vapor interface in the evaporator region ([2] notes that this could be estimated as being equal to $R_{\text {eff }}$ ).

\subsubsection{Frozen Startup Limit Number}

The heat pipe limits described in Section 2.5.1 represent maximum heat rates that can be sustained at a given operating temperature. The limit described in this section is of a different nature, representing either success or failure of a heat pipe to start up from a frozen state.

The startup of a high-temperature heat pipe from a frozen state proceeds as follows. First, heat is applied in the evaporator region, eventually producing a melt front that propagates inward radially toward the vapor core. Assuming a lack of noncondensable gases in the core, a vacuum exists until the melt front reaches the wick surface, at which time vaporization begins to occur. As vaporization continues, eventually a continuum vapor phase develops instead of just a rarefied gas, and this continuum vapor front gradually moves down the heat pipe toward the condenser end, along with the melt front in the liquid phase. Vapor condenses partly on the already-melted working fluid and partly on the still-frozen working fluid. Upon condensing on the still-frozen working fluid, heat is transferred to the solid phase and, if close to the melting point, may even supply enough energy to melt it. Otherwise, the condensed vapor ends up freezing on this surface. If this 
freezing rate exceeds the rate at which the melt rate propagates down the heat pipe, the frozen startup limit (FSL), as defined by Faghri [2], is encountered, and the heat pipe fails due to an insufficient liquid return to the evaporator, since it is frozen elsewhere.

Cao and Faghri quantitatively investigated this limit using an FSL number, defined as follows:

$$
\mathrm{FSL}=\frac{\rho_{\ell} \Delta h_{\ell v} A_{\ell}}{\Delta E_{\text {melt }}^{\prime}}
$$

where $\rho_{\ell}$ is the liquid density, $\Delta h_{\ell v}$ is the latent heat of vaporization, $A_{\ell}$ is the cross-sectional area of the liquid phase, and $\Delta E_{\text {melt }}^{\prime}$ is the energy change per unit length required to melt the working fluid from the initial temperature $T_{0}$ :

$$
\Delta E_{\text {melt }}^{\prime}=C^{\prime}\left(T_{\text {melt }}-T_{0}\right)+\rho_{\ell} \Delta h_{\text {fus }} A_{\ell},
$$

where $T_{\text {melt }}$ is the melting temperature, $\Delta h_{\text {fus }}$ is the latent heat of fusion, and $C^{\prime}$ is the heat capacity per unit length:

$$
C^{\prime}=A_{\text {clad }} \rho_{\text {clad }} c_{p, \text { clad }}+A_{\ell} \rho_{\ell} c_{p, \ell}+(1-\epsilon) A_{\text {wick }} \rho_{\text {wick }} c_{p, \text { wick }} .
$$

Here, the subscript "clad" denotes quantities associated with the cladding, $\ell$ with the liquid phase of the working fluid, and "wick" with the wick material. If FSL $\geq 1$, the heat pipe is predicted to start up successfully; otherwise, it is predicted to encounter the frozen startup limit.

Interestingly, the limit does not depend on the heat rate applied to the heat pipe; rather, it depends only on the following:

- The initial temperature

- The thermodynamic properties of the working fluid

- The thermal properties of the wick and cladding

- The fill level of the working fluid

- The heat pipe geometry.

The lack of dependence on the heat rate is due to both of the effects (melt propagation and refreezing of condensing vapor) being proportional to the heat rate and thus cancelling when computing the ratio.

Unfortunately, there are only a few examples in the literature that report usage of the above FSL criteria, for which a comparison can be made to the implementation in Sockeye. These comparisons are summarized in Table 2, where $\mathrm{FSL}_{\text {Sockeye }}$ is the value that Sockeye computes, $\mathrm{FSL}_{\text {ref }}$ is the value reported in the given reference, and $r$ is the relative difference between these numbers, expressed as a percentage:

$$
r \equiv 100 \frac{\mathrm{FSL}_{\text {Sockeye }}-\mathrm{FSL}_{\mathrm{ref}}}{\mathrm{FSL}_{\mathrm{ref}}} .
$$

While the comparison to the Dickinson references shows good agreement, the comparison to the Cao and Faghri reference indicates a significant discrepancy. Some possibilities for this discrepancy are as follows:

- Different thermodynamic property values used

- Different initial temperature used

- Different geometrical approximations (e.g., area of thin annulus) 
Table 2: Comparison of computed FSL values with reference values.

\begin{tabular}{lccc}
\hline Case & FSL $_{\text {Sockeye }}$ & FSL $_{\text {ref }}$ & Relative Difference (\%), $r$ \\
\hline Cao and Faghri [19] & 2.32 & 1.46 & 58.9 \\
Dickinson, Annular [20] & 11.5 & 12.3 & 6.50 \\
Dickinson, Homogeneous [20] & 6.8 & 6.7 & 1.5 \\
\hline
\end{tabular}

- Cao and Faghri's neglect of unfilled wick heat capacity

- Different porosity calculation.

While this discrepancy is significant, it is worthwhile to note that the conclusions between the Sockeye and reference calculations are the same; in both cases, FSL $\geq 1$, and thus the startup of the heat pipe is predicted to be successful. Unfortunately, there are no cases in the literature that demonstrate that FSL $<1$ for a heat pipe configuration for which startup failed.

\section{MODEL IMPROVEMENTS}

This section describes a number of improvements to existing models that customers have requested and is organized as follows. Section 3.1 describes improvements made to the capillary pressure model, Section 3.2 describes improvements made to pressure loss terms in both the liquid and vapor phases, and Section 3.3 describes improvements made to wall heat transfer and the addition of terms to enable wall boiling.

\subsection{Capillary Pressure}

Capillary pressure is responsible for a heat pipe's ability to sustain its operation by circulating the working fluid, possibly against the force of gravity. Sockeye's flow model is classified as a two-pressure model; that is, each of its phases (liquid and vapor) has its own pressure, which is not necessarily equal to that of the other phase. These pressures are independent, but pressure relaxation terms drive the phase boundaries to try to match the following condition:

$$
\Delta p_{\text {cap }}=p_{v}-p_{\ell}
$$

where $\Delta p_{\text {cap }}$ is the capillary pressure, $p_{v}$ is the vapor pressure, and $p_{\ell}$ is the liquid pressure. For example, if $\Delta p_{\text {cap }}$ exceeds the local pressure difference $p_{v}-p_{\ell}$, the liquid phase will attempt to expand. An accurate prediction of $\Delta p_{\text {cap }}$ thus governs important fluid dynamics that determine if dryout will ultimately occur in the heat pipe under a certain set of conditions.

This section describes improvements made to the calculation of capillary pressure and is organized as follows. Section 3.1.1 describes major changes made the "excess-liquid" regime that prevent the prediction of negative capillary pressure, and Section 3.1.2 describes the new ability to fine-tune the capillary pressure relationship as a function of wick saturation, using experimentally determined data. 


\subsubsection{Excess Liquid Regime}

The nature of capillary pressure is complex, depending on factors such as the combination of working fluid and wick material, the wick structure, temperature, wick surface conditions, and local, dynamic conditions. The prediction accuracy of capillary pressure is important to correctly predict the capillary limit and the circulation of the working fluid.

Sockeye calculates capillary pressure from the void fraction solution, in conjunction with geometrical information about the wick provided by the user. The main assumption is that each void fraction solution value maps uniquely to a liquid-vapor interface configuration, which consists of the radial position of the interface and the curvature of the interface in each pore of the wick at that radial position, if the interface is in the wick. If in the wick, there is capillary pressure, obeying the following relation:

$$
\Delta p_{\text {cap }}=\frac{2 \sigma}{R_{\text {men }}}
$$

where $R_{\text {men }}$ is the curvature radius of the meniscus of the liquid-vapor interface. This section describes some changes made to the existing assumptions regarding the shape of the interface in certain ranges of the void fraction.

Figure 11 gives an illustration of the liquid-vapor interface location and shape at a number of bounding

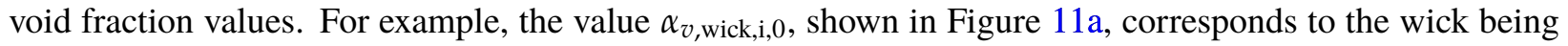
perfectly saturated with working fluid, with a flat liquid-vapor interface. The value $\alpha_{v, \text { wick,i,+ }}$ corresponds to the radial interface position still being at the inner surface of the wick but this time having a fully hemispherical, concave interface in each pore. Similarly, $\alpha_{v, \text { wick,o,+ }}$ again corresponds to having a fully hemispherical, concave interface in each pore but this time having the radial position of the interface at the outer surface of the wick. For $\alpha_{v}<\alpha_{v \text {,wick,i, } 0}$ or $\alpha_{v}>\alpha_{v \text {, wick,o,+ }}$, the interface is assumed to be flat. For $\alpha_{v \text {,wick,i, } 0}<\alpha_{v}<\alpha_{v \text {,wick,i,+ }}$, the interface curvature varies from flat to fully hemispherical, depending on $\alpha_{v}$. For $\alpha_{v \text {,wick,o,- }}<\alpha_{v}<\alpha_{v \text {,wick,o,+ }}$, we assume the interface is a fully hemispherical, concave interface in each pore.

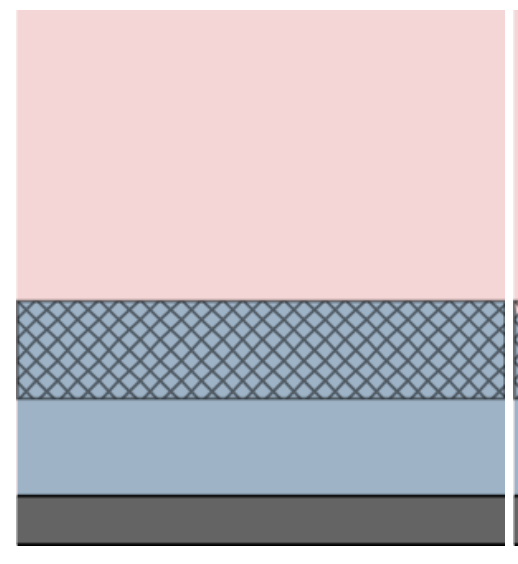

(a) $\alpha_{v, \text { wick,i,0 }}$

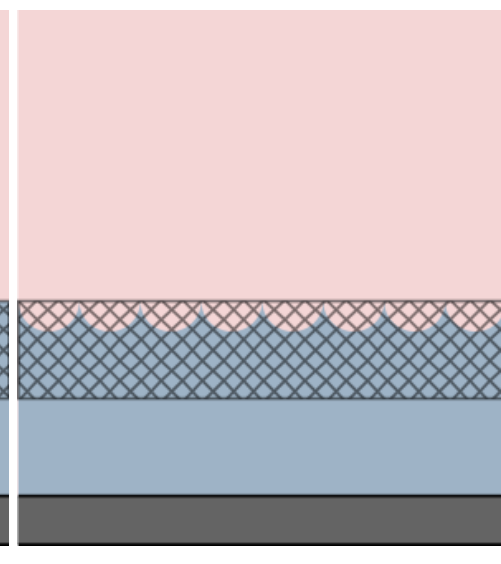

(b) $\alpha_{v, \text { wick,i,+ }}$

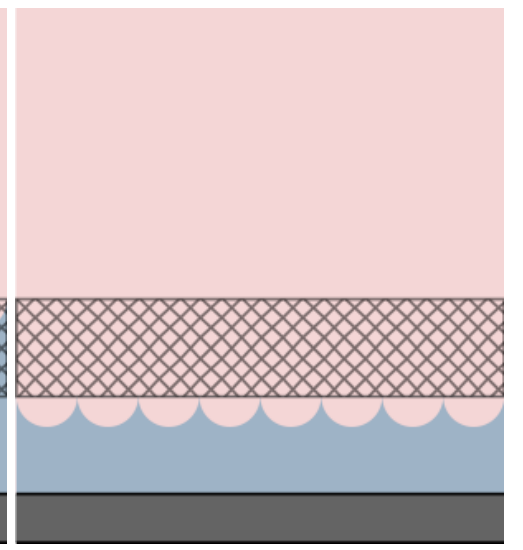

(c) $\alpha_{v, \text { wick,o,+ }}$

Figure 11: Illustration of liquid-vapor interface vs. void fraction at bounding values.

This picture corresponds to the current assumptions of the liquid-vapor interface. The old assumptions

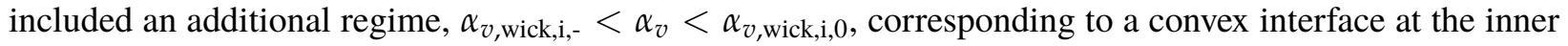


radial surface of the wick, where $\alpha_{v \text {, wick,i,- }}$ corresponds to a fully hemispherical, convex interface in each pore. This convexity flips the sign in (31), leading to a negative capillary pressure. A negative capillary pressure is not necessarily unphysical; some fluid-surface combinations are known to produce negative capillary pressures in some conditions. Unfortunately, experimental data for pressure measurements inside heat pipes is nonexistent, so the observation of no negative capillary pressures inside a heat pipe is based solely on theoretical analysis, which is often restricted to arguments relying on steady conditions. Nevertheless, the prevailing expectation is that heat pipes do not feature negative capillary pressure, which motivated the change in assumption described here.

Figure 12 shows results corresponding to the old capillary pressure model, featured in [21]. The liquid pressure exceeds the vapor pressure when $\alpha_{v}<\alpha_{v \text {,wick,i, } 0}$, due to the assumption that the interface is convex in this condition, yielding a negative capillary pressure. In contrast, Figure 13 shows results corresponding to the new capillary pressure model. The liquid pressure never exceeds the vapor pressure, due to the assumption of no convex interfaces and thus no negative capillary pressures.

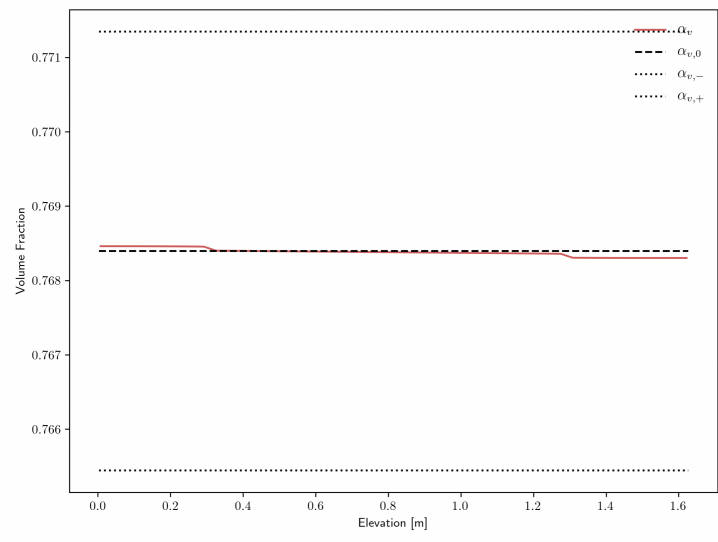

(a) Void fraction solution.

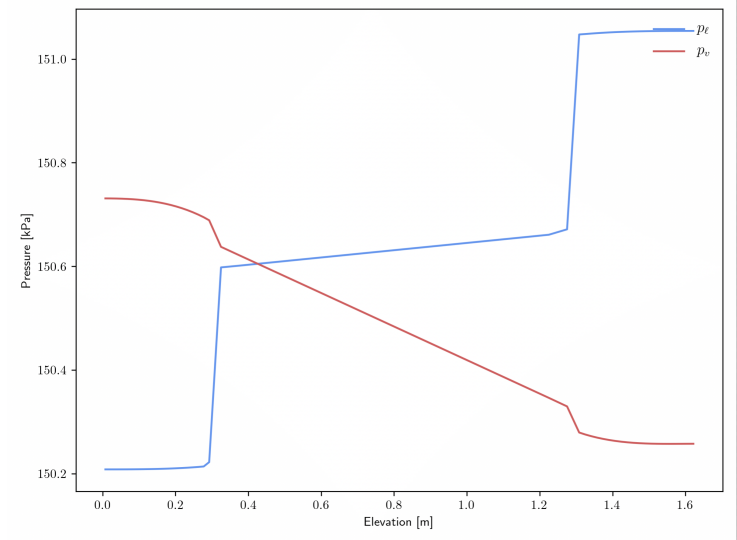

(b) Pressure solutions.

Figure 12: Void fraction and pressure solutions using the old excess liquid capillary pressure relationship.

\subsubsection{Saturation Dependence}

This section describes a model, developed in collaboration with Argonne National Laboratory, which allows the user to customize the capillary pressure relationship.

Experimental evidence indicates a dependence of capillary pressure in a porous structure on its saturation with the wetting phase [22-24]. This is believed to be due to a number of factors that affect the effective pore radius as the fluid moves through the structure layers, such as the randomness of pore distributions or even by manufacturing design. For example, some heat pipe wicks of the wrapped screen variety use a composite design where different layers have different mesh sizes, which can prove advantageous for capillary pressure [25]. Another cause of variable capillary pressure in wrapped screen wicks is the compression between layers. In general, these features are all very complex, and representative wick samples should be used to experimentally determine the saturation relationship of capillary pressure when possible.

In the scientific discipline of porous flow, the saturation, $\zeta$, is defined as the fraction of void volume of the porous structure that is occupied by the wetting phase. In this case, the wick is the porous structure, and 


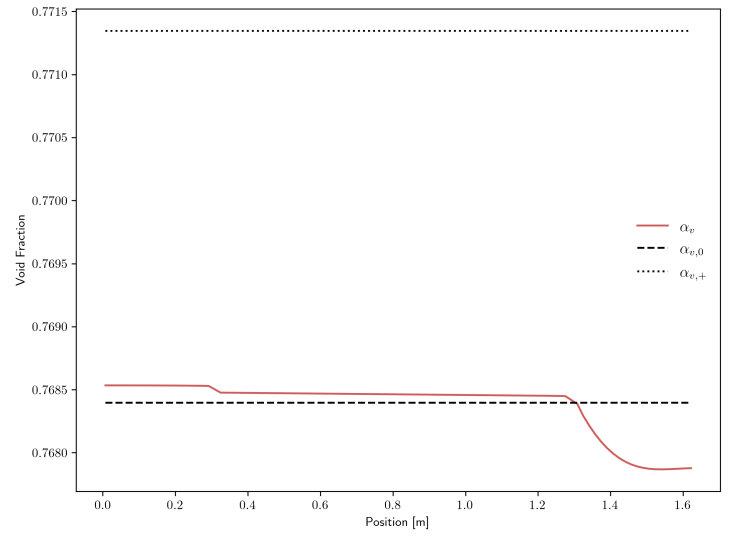

(a) Void fraction solution.

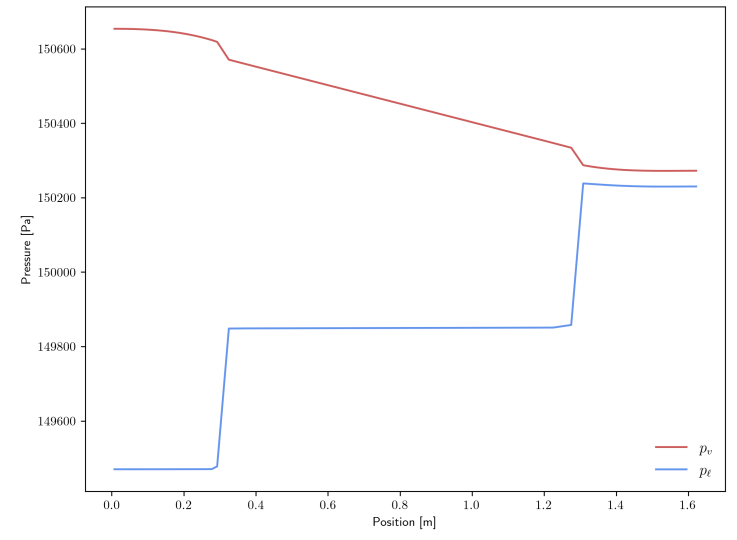

(b) Pressure solutions.

Figure 13: Void fraction and pressure solutions using the new excess liquid capillary pressure relationship.

the wetting phase is the liquid phase of the working fluid. Sockeye considers wicks having an annular cross section, whose area is denoted by $A_{\text {wick }}$ and porosity by $\epsilon$, thus making its void area $\epsilon A_{\text {wick. }}$ Denoting the cross-sectional area of the liquid phase within the wick by $A_{\ell \text {, wick }}$, the wick saturation is defined to be

$$
\zeta \equiv \frac{A_{\ell \text {,wick }}}{\epsilon A_{\text {wick }}}
$$

The term $A_{\ell \text {,wick }}$ is computed using the void fraction solution $\alpha_{v}$ and wick geometrical parameters.

There are a number of formulations of capillary pressure vs. saturation, but one of the most prominent is the Leverett "J-function," which has its origins in the petroleum industry [22]. This formulation gives a dimensionless pressure $J$ as a function of saturation:

$$
J(\zeta)=\frac{\sqrt{\frac{K}{\epsilon}}}{\sigma \cos \theta} \Delta p_{\text {cap }},
$$

where $K$ is the wick permeability, $\sigma$ is the surface tension, $\theta$ is the contact angle, and $\Delta p_{\text {cap }}$ is the capillary pressure. This function is determined experimentally and then inverted to give the capillary pressure as a function of saturation.

This J-function was implemented in Sockeye as an optional user parameter, defaulting to a value of unity, which corresponds to no saturation dependence. An example of this function is shown in Figure 14.

\subsection{Pressure Losses}

The maximum power that a heat pipe can sustain at a given operating temperature is determined by a number of limits. As shown in Figure 10, for a typical heat pipe, at low temperature, the viscous and sonic limits are limiting the heat rate. During normal operation, the temperature will be higher, and the operation of the heat pipe will be limited by the capillary limit in most cases. Thus, the performance of the heat pipe is tightly linked to the capillary limit of the heat pipe, which occurs when the pumping rate of the wick cannot provide sufficient liquid to the evaporator section [2] and results in dryout at the evaporator. In other terms, it 


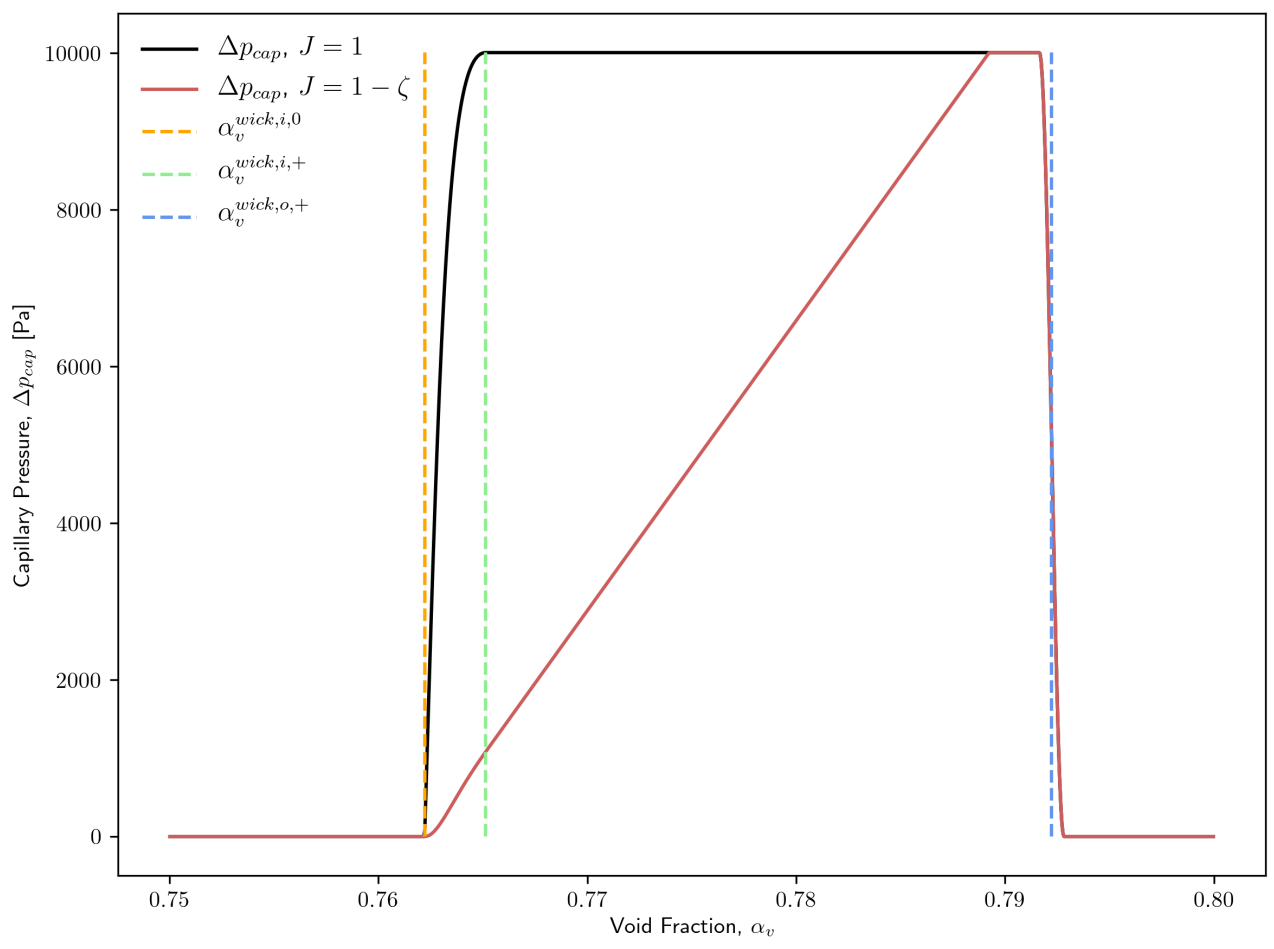

Figure 14: Capillary pressure example using the $J$-function $J(\zeta)=1-\zeta$.

occurs when the sum of the liquid and vapor pressure drops exceeds the maximum capillary pressure of the wick. Thus, it is important to properly evaluate the pressure losses due to wall friction and the porous flow in the wick to capture the capillary limit. In particular, high performance heat pipes will have an annular wick structure with a small pore radius to obtain a higher maximum capillary pressure, leading to a small permeability. The axial liquid velocity will exhibit strong radial variations, with higher velocities in the annulus and smaller velocities in the wick. These multidimensional effects can be captured by the Sockeye 1D equation system by using appropriate source terms in the momentum equations. Therefore, closures for the liquid and vapor friction factors have been added to improve the accuracy of pressure loss predictions.

\subsubsection{Liquid Phase}

For a homogeneous wick in contact with the cladding, the axial flow of the liquid phase through a porous wick structure yields a pressure loss that can be approximated using the Darcy equation:

$$
\frac{d p_{\ell}}{d x}=-\frac{\mu_{\ell} u_{\ell}}{K}
$$

where $\mu_{\ell}$ is the liquid viscosity, $u_{\ell}$ is the liquid velocity in the porous structure, and the $K$ is the permeability of the wick.

When the wick structure is not flush to the inner cladding surface, there are two flow paths for the liquid to travel back from the condenser region to the evaporator region: the wick and the annulus. Thus, the liquid pressure losses are a combination of the losses due to the porous flow in the wick and due to the wall friction in the annulus. The total liquid mass flow rate is split so that the pressure losses in the wick structure and in the annulus are equal, leading to different average velocities in each flow path. 
The wall friction in the annulus is due to the inner wall of the heat pipe and wick structure. It is evaluated using a Darcy friction loss factor:

$$
\begin{aligned}
\left(\frac{d p_{\ell}}{d x}\right)_{\mathrm{ann}} & =-\frac{1}{2} f_{\mathrm{D}, \ell} \frac{\rho_{\ell}\left|u_{\mathrm{ann}, \ell}\right| u_{\mathrm{ann}, \ell}}{D_{\mathrm{h}, \mathrm{ann}, \ell}} \\
& =-\frac{1}{2} f_{\mathrm{D}, \ell} \frac{\left|\dot{m}_{\mathrm{ann}, \ell}\right| \dot{m}_{\mathrm{ann}, \ell}}{\rho_{\ell} A_{\mathrm{ann}, \ell}^{2} D_{\mathrm{h}, \mathrm{ann}, \ell}}=-C_{a}\left|\dot{m}_{\mathrm{ann}, \ell}\right| \dot{m}_{\mathrm{ann}, \ell},
\end{aligned}
$$

where $D_{\mathrm{h}, \text { ann, } \ell}$ is the hydraulic diameter in the annulus, $u_{\mathrm{ann}, \ell}$ is the average velocity of the liquid phase in the annulus, and $C_{a}$ is defined as

$$
C_{a}=\frac{f_{\mathrm{D}, \ell}}{2 \rho_{\ell} A_{\mathrm{ann}, \ell}^{2} D_{\mathrm{h}, \mathrm{ann}, \ell}} .
$$

The hydraulic diameter of the annulus depends on the location of the interface between the liquid and the vapor phases. If the interface is located in the wick structure or in the vapor core,

$$
D_{\mathrm{h}, \mathrm{ann}, \ell}=D_{\mathrm{hp}, \mathrm{i}}-D_{\text {wick,o }} .
$$

If the interface is located in the annulus, there is no contact between the liquid phase and the wick structure and the hydraulic diameter is calculated the liquid thickness. The liquid thickness on the heat pipe wall is calculated as

$$
\delta=\frac{D_{\mathrm{hp}, \mathrm{i}}}{2}\left(1-\sqrt{1-\alpha_{\ell}}\right) .
$$

The hydraulic diameter is then

$$
D_{\mathrm{h}, \mathrm{ann}, \ell}=2 \delta\left(2-\frac{2 \delta}{D_{\mathrm{hp}, \mathrm{i}}}\right) .
$$

If the user does not provide a friction factor for the liquid phase, the friction factor is determined assuming a laminar flow in the annulus [26]:

$$
f_{\mathrm{D}}=\frac{24}{\operatorname{Re}}\left(\frac{D_{\text {wick }, \mathrm{o}}}{D_{\text {hp,i }}}\right)^{0.035}
$$

The porous losses in the wick can be expressed using Equation (34):

$$
\begin{aligned}
\left(\frac{d p_{\ell}}{d x}\right)_{\text {wick }} & =-\frac{\mu_{\ell} u_{\text {wick }, \ell}}{K} \\
& =-\frac{\mu_{\ell} \dot{m}_{\text {wick }, \ell}}{\rho_{\ell} A_{\text {wick }, \ell} K}=-C_{w} \dot{m}_{\text {wick }, \ell},
\end{aligned}
$$

where $u_{\text {wick }, \ell}$ is the average velocity in the wick, $\dot{m}_{\text {wick }, \ell}$ is the liquid mass flow rate in the wick structure, and $C_{w}$ is defined as

$$
C_{w}=\frac{\mu_{\ell}}{\rho_{\ell} A_{\text {wick }, \ell} K} .
$$

By equating the pressure loss in each flow path, the mass flow rates can be expressed as

$$
\begin{aligned}
\left|\dot{m}_{\mathrm{ann}, \ell}\right| & =\frac{C_{w}}{2 C_{a}}\left(-1+\sqrt{1+4 \frac{C_{a}}{C_{w}}\left|\dot{m}_{\ell}\right|}\right), \\
\left|\dot{m}_{\text {wick }, \ell}\right| & =\left|\dot{m}_{\ell}\right|-\left|\dot{m}_{\text {ann }, \ell}\right|
\end{aligned}
$$


where $\dot{m}_{\ell}$ is the total liquid mass flow rate.

The momentum source term for the combined porous and wall friction is then

$$
-F_{\ell} A=-C_{a}\left|\dot{m}_{\mathrm{ann}, \ell}\right| \dot{m}_{\mathrm{ann}, \ell} \alpha_{\ell} A .
$$

If there is no liquid in the wick structure, all the flow goes through the annulus, and only the wall friction losses on the heat pipe inner wall are applied.

Note that this derivation assumes that there is no liquid flow in the core of the heat pipe. This might not be true, especially in the condenser where a liquid pool can be formed, but this assumption is used only to derive the pressure losses in the liquid phase. In addition, if a liquid pool is present, the velocities are small and the resulting pressure losses would be small as well. It is also assumed that the flow is co-current in each flow path.

\subsubsection{Vapor Phase}

The pressure losses in the vapor phase are caused by the wall friction on the inner wall of the wick structure. Using a Darcy friction factor $f_{\mathrm{D}, v}$, the friction force density $F_{v}^{\text {wall }}$ in the vapor momentum conservation equation is the following:

$$
F_{v}^{\text {wall }}=\frac{1}{2} \frac{f_{\mathrm{D}, v} \alpha_{v} \rho_{v}\left|u_{v}\right| u_{v}}{D_{\mathrm{h}, v}}
$$

where $D_{\mathrm{h}, v}$ is the hydraulic diameter associated with the vapor phase

$$
D_{\mathrm{h}, \mathrm{v}}=D_{\text {wick,i }} \text {. }
$$

If the user does not provide a friction factor for the vapor phase, the friction factor is determined as follows. For laminar flow $\left(\operatorname{Re}_{v}<2000\right)$, the Hagen-Poiseuille equation is used, and, for turbulent flow $\left(\operatorname{Re}_{v} \geq 2000\right)$, the Blasius relation is used [2]:

$$
f_{\mathrm{D}}=\left\{\begin{array}{l}
\frac{64}{\operatorname{Re}_{v}} \quad \operatorname{Re}_{v}<2000 \\
\frac{0.316}{\operatorname{Re}_{v}^{1 / 4}} \operatorname{Re}_{v} \geq 2000
\end{array} .\right.
$$

A smooth transition is implemented between $\operatorname{Re}_{v}=1500$ and $\operatorname{Re}_{v}=2500$ to alleviate the discontinuity at $\operatorname{Re}_{v}=2000$. This is shown in Figure 15.

\subsection{Wall Heat Transfer}

Heat is radially transferred to the heat pipe on the evaporator side and rejected on the condenser side through heat conduction through the cladding. Thus, the heat transfer between the cladding inner wall and the working fluid is key to properly capture the thermal resistance and predict the working fluid temperature distribution. Furthermore, high heat flux at startup can trigger wall boiling, which can significantly affect the performance of the heat pipe by increasing the vapor generation. In addition, the bubbles generated on the wall interact in the wick with the meniscus, disturbing the action of the capillary pressure. The wall 


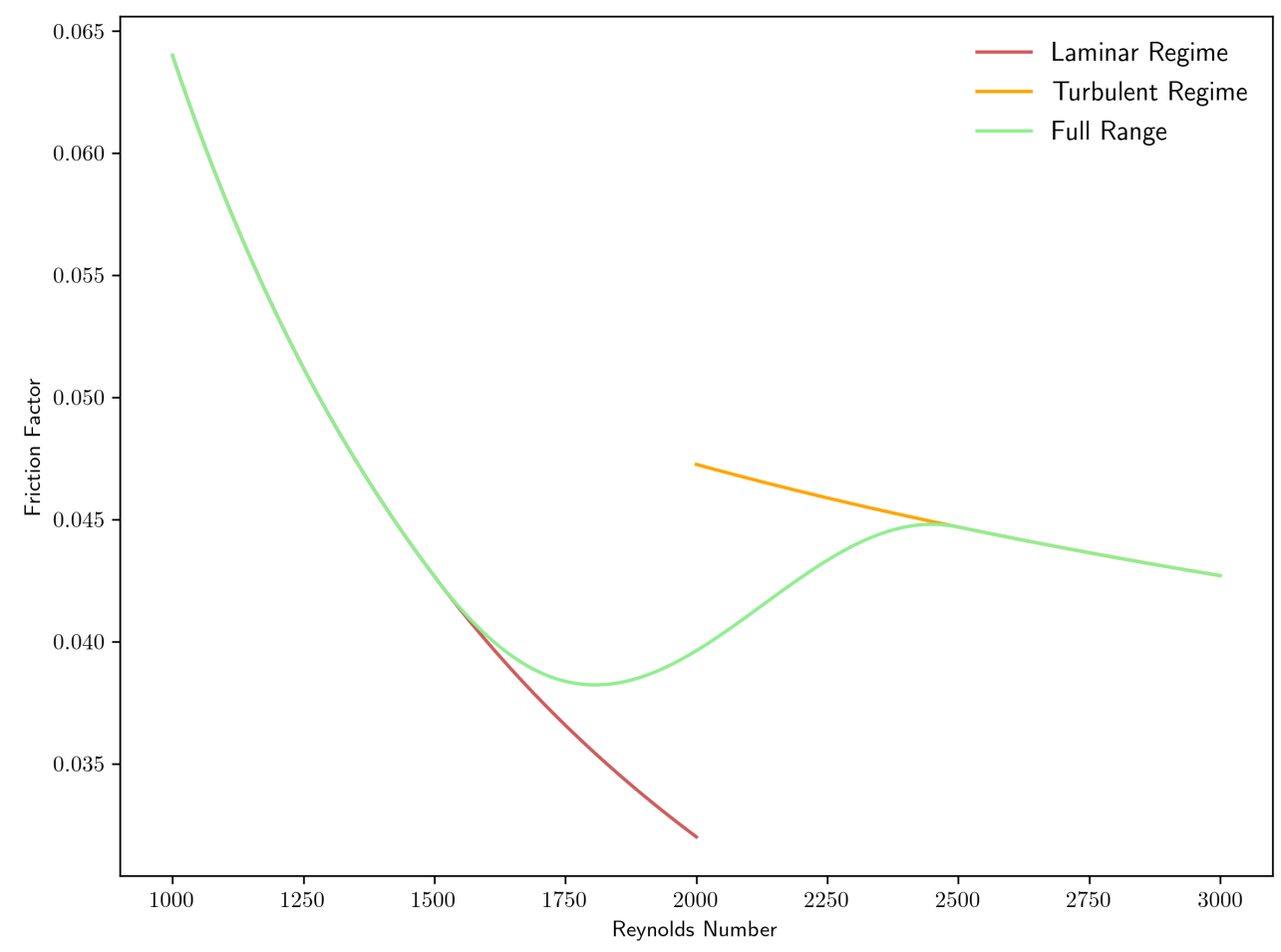

Figure 15: Vapor friction factor transition at $\operatorname{Re}_{v}=2000$.

heat transfer has been further developed to include flow regime dependent closures for the wall heat transfer coefficient and a wall boiling model to calculate the mass transfer at the wall.

The wall heat transfer model is described below. The wall heat flux is partitioned into a portion that may go directly to convective heat transfer to the vapor phase and a portion that is available to both convectively heat the liquid phase and generate vapor via wall boiling. This partitioning is specified with a simple function $\kappa$, which is the fraction of the wall surface in contact with the vapor phase. The portion of the wall heat flux available to convectively heat the liquid phase and generate vapor is further partitioned into a portion that may convectively heat the liquid phase and a portion that goes toward the generation of vapor. This partitioning is specified with a simple function $f_{\text {boil }}$. Rendering this into equation form,

$$
\begin{gathered}
q_{\text {wall,total }}=q_{\text {wall } \rightarrow \ell}^{\text {conv }}+q_{\text {wall } \rightarrow \ell}^{\text {boil }}+q_{\text {wall } \rightarrow v}, \\
q_{\text {wall } \rightarrow \ell}^{\text {boil }}=f_{\text {boil }} \mathcal{H}_{\text {wall }, \ell}\left(T_{\text {wall }}-T_{\ell}\right) \kappa, \\
q_{\text {wall } \rightarrow \ell}^{\text {conv }}=\left(1-f_{\text {boil }}\right) \mathcal{H}_{\text {wall }, \ell}\left(T_{\text {wall }}-T_{\ell}\right) \kappa, \\
q_{\text {wall } \rightarrow v}=\mathcal{H}_{\text {wall }, v}\left(T_{\text {wall }}-T_{v}\right)(1-\kappa),
\end{gathered}
$$

where $T_{\text {wall }}$ is the inner cladding wall temperature, $T_{\ell}$ and $T_{v}$ are the liquid and vapor bulk temperatures, and $\mathcal{H}_{\text {wall }, \ell}$ and $\mathcal{H}_{\text {wall }, v}$ are the liquid and vapor heat transfer coefficients.

The mass flux of vapor generated by boiling at the wall due to wall heat flux is

$$
\Gamma_{\ell \rightarrow v}^{\text {wall }}=\frac{q_{\mathrm{wall} \rightarrow \ell}^{\text {boil }}}{h_{v}^{\text {int }}-h_{\ell}},
$$


where $h_{v}^{\mathrm{int}}-h_{\ell}$ represents the change in specific enthalpy required for vaporization.

The wall heat transfer coefficients $\mathcal{H}_{\text {wall, } \ell}$ and $\mathcal{H}_{\text {wall }, v}$ and the coefficients $\kappa$ and $f_{\text {boil }}$ are determined depending on the heat transfer mode. To determine the heat transfer mode, the inner cladding wall temperature $T_{\text {wall }}$ is compared with the temperature of onset nucleate boiling.

\section{Temperature of onset of nucleate boiling}

The temperature of onset of nucleate boiling, $T_{\mathrm{ONB}}$, is calculated with the same approach as the boiling limit given by [2]:

$$
T_{\mathrm{ONB}}=T_{v}+\frac{2 \sigma T_{v}}{h_{\text {lat }} \rho_{v}}\left(\frac{1}{R_{b}}-\frac{1}{R_{\mathrm{men}}}\right)
$$

where $h_{\text {lat }}$ is the latent heat of evaporation, $R_{b}$ is the radius of the bubbles generating at the wall, and $R_{\text {men }}$ is the meniscus radius. The bubble radius is difficult to estimate as it depends on the surface conditions. For simplicity, we set it to $1 \mu \mathrm{m}$.

Convective liquid heat transfer Convective liquid heat transfer occurs if the void fraction is lower than 0.99999 and the wall temperature is lower than the temperature of onset of nucleate boiling. The heat flux partitioning coefficient $\mathcal{K}$ is set to 1 . There is no wall boiling, so $f_{\text {boil }}$ is set to 0 .

The liquid heat transfer coefficient is computed by

$$
\mathcal{H}_{\text {wall }, \ell}^{\mathrm{FC}}=\mathrm{Nu}_{\ell} \frac{k_{\ell}}{D_{h p, i}},
$$

where the Nusselt number is calculated using the Skupinski correlation [27]:

$$
\mathrm{Nu}_{\ell}=4.82+0.0185 \cdot \mathrm{Pe}_{\ell}^{0.827},
$$

with Pe being the Peclet number. In this heat transfer mode, no heat is transferred to the vapor. Thus, the vapor heat transfer coefficient $\mathcal{H}_{\text {wall, } v}$ is set to 0 .

Single phase vapor heat transfer This regime occurs when the void fraction is higher than 0.99999 . Only vapor is in contact with the wall. In these conditions, the heat flux partitioning coefficient $\kappa$ is set to 0 . There is no wall boiling, so $f_{\text {boil }}$ is set to 0 . The vapor heat transfer coefficient $\mathcal{H}_{\text {wall }, v}$ is calculated similarly to the liquid heat transfer coefficient using the Skupinski correlation:

$$
\mathcal{H}_{\text {wall }, v}=\mathrm{Nu}_{v} \frac{k_{v}}{D_{h p, i}},
$$

where the Nusselt number is:

$$
\mathrm{Nu}_{v}=4.82+0.0185 \cdot \mathrm{Pe}_{v}^{0.827}
$$

Nucleate boiling For nucleate boiling, the wall is covered by the liquid phase, so $\kappa$ and $\mathcal{H}_{\text {wall, } v}$ are set to 0 . The heat flux is the convective heat flux plus pool boiling heat flux:

$$
\mathcal{H}_{\mathrm{wall}, \ell}^{\mathrm{NB}}=\mathcal{H}^{\mathrm{FC}}+\mathcal{H}^{\mathrm{PB}}\left(T_{\mathrm{wall}}\right)-\mathcal{H}^{\mathrm{PB}}\left(T_{\mathrm{ONB}}\right),
$$

where $\mathcal{H}^{\mathrm{FC}}$ is the convective heat transfer coefficient calculated with the Skupinski correlation and $\mathcal{H}^{\mathrm{PB}}$ is the pool boiling heat transfer coefficient, which is calculated using the correlation from Shah [28]:

$$
\mathcal{H}^{\mathrm{PB}}=C q^{0.7} p_{r}^{m}
$$


with $p_{r}$ being the ratio of the liquid pressure to the critical pressure and $C$ and $m$ being constants (13.7 and 0.22 , respectively, for $p_{r}<0.001 ; 6.9$ and 0.12 for $p_{r}>0.001$ ).

The boiling fraction is then calculated by:

$$
f_{\text {boil }}=\frac{\mathcal{H}^{\mathrm{PB}}\left(T_{\mathrm{wall}}\right)-\mathcal{H}^{\mathrm{PB}}\left(T_{\mathrm{ONB}}\right)}{\mathcal{H}_{\mathrm{wall}, \ell}^{\mathrm{NB}}} .
$$

An example has been added to demonstrate the wall boiling capability. A very high heat flux is applied to the evaporator and the temperature of the outer cladding wall is maintained to a constant value. As shown in Figure 16a, the temperature on the inner wall of the cladding is rising above the temperature of onset of nucleate boiling. The result is that the wall boiling fraction becomes greater than 0 in this region and vapor is generated at the wall, as shown in Figure 16b. To compensate for the added vapor generation, the condensation rate is higher in the condenser region. Note that this setup has been designed to demonstrate the wall boiling capability and is not representative of a realistic configuration.

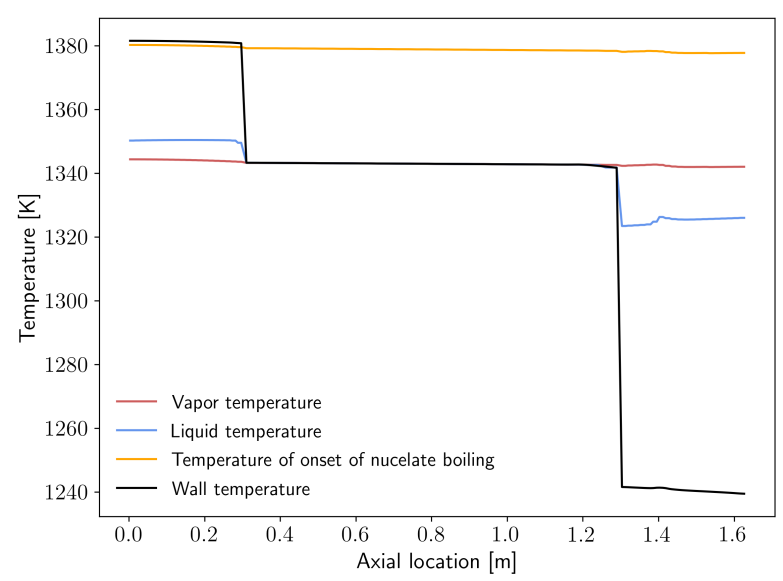

(a) Temperature distributions.

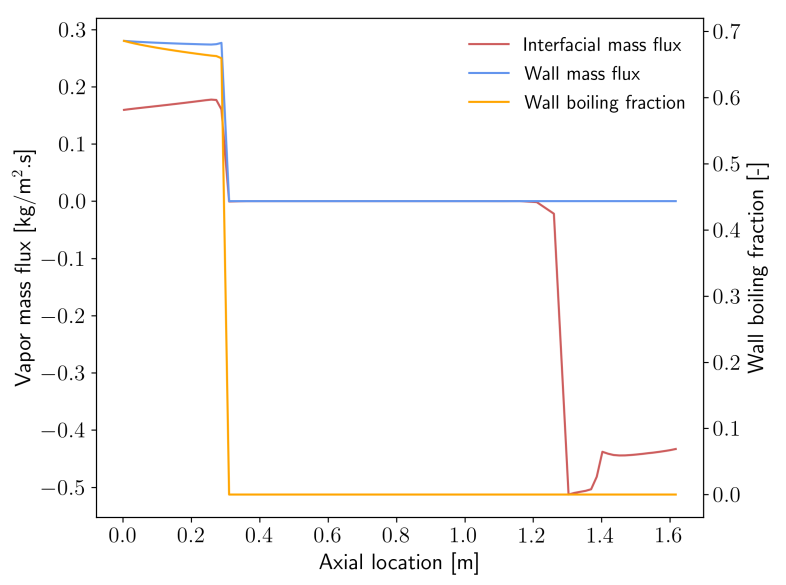

(b) Wall boiling fraction and interfacial and wall mass fluxes.

Figure 16: Temperature and mass transfers distributions during wall boiling.

\section{USER DOCUMENTATION AND TRAINING}

User documentation and training resources are essential for users learning to use the software. Section 4.1 describes a number of user examples that were added, and Section 4.2 describes some discretization documentation that was added to the theory manual.

\subsection{User Examples}

One of the main requests from users is for usage examples. Many examples are available in the distributed code repository. Each example demonstrates a specific capability of Sockeye with the problem definition and corresponding post-processing tools. Specific examples requested by Westinghouse are described in this section. 


\subsubsection{Custom Friction Closures}

Closures in Sockeye define the correlations used to calculate the following quantities: liquid and vapor friction factors, liquid and vapor heat transfer coefficients, liquid and vapor interfacial heat transfer coefficients, liquid and vapor wall contact fractions, wall temperature, interfacial area density, and capillary pressure. The default closures used in Sockeye are meant to be as widely applicable as possible, but many of these relations can be improved if users have performed experiments for their specific heat pipe designs. Sockeye allows users to replace default closures for maximum flexibility, in many cases without requiring any code development.

One example was added as a verification of the custom closures technique; the example disables the default closures and then replicates them in the input file in the same way that a user would provide their own closures, and the solution is verified to be identical to that produced by an input file using the default closures.

Another example was added to demonstrate an actual custom closure, in this case, the friction factor for the vapor phase. The vapor friction factor was chosen to be an arbitrary function of the Reynolds number, since a dependence on the Reynolds number is a common occurrence for friction factors:

$$
f_{D \text { vapor }}=a+b \cdot \operatorname{Re}_{\text {vapor }}^{c},
$$

where $a, b$, and $c$ are set to $0.02,0.1$, and -0.25 , respectively. The resulting friction factor is plotted against the vapor Reynolds number in Figure 17 and is compared to the analytical definition given by Equation (60) and the friction factor calculated by the default Sockeye closure, described in Section 3.2.2.

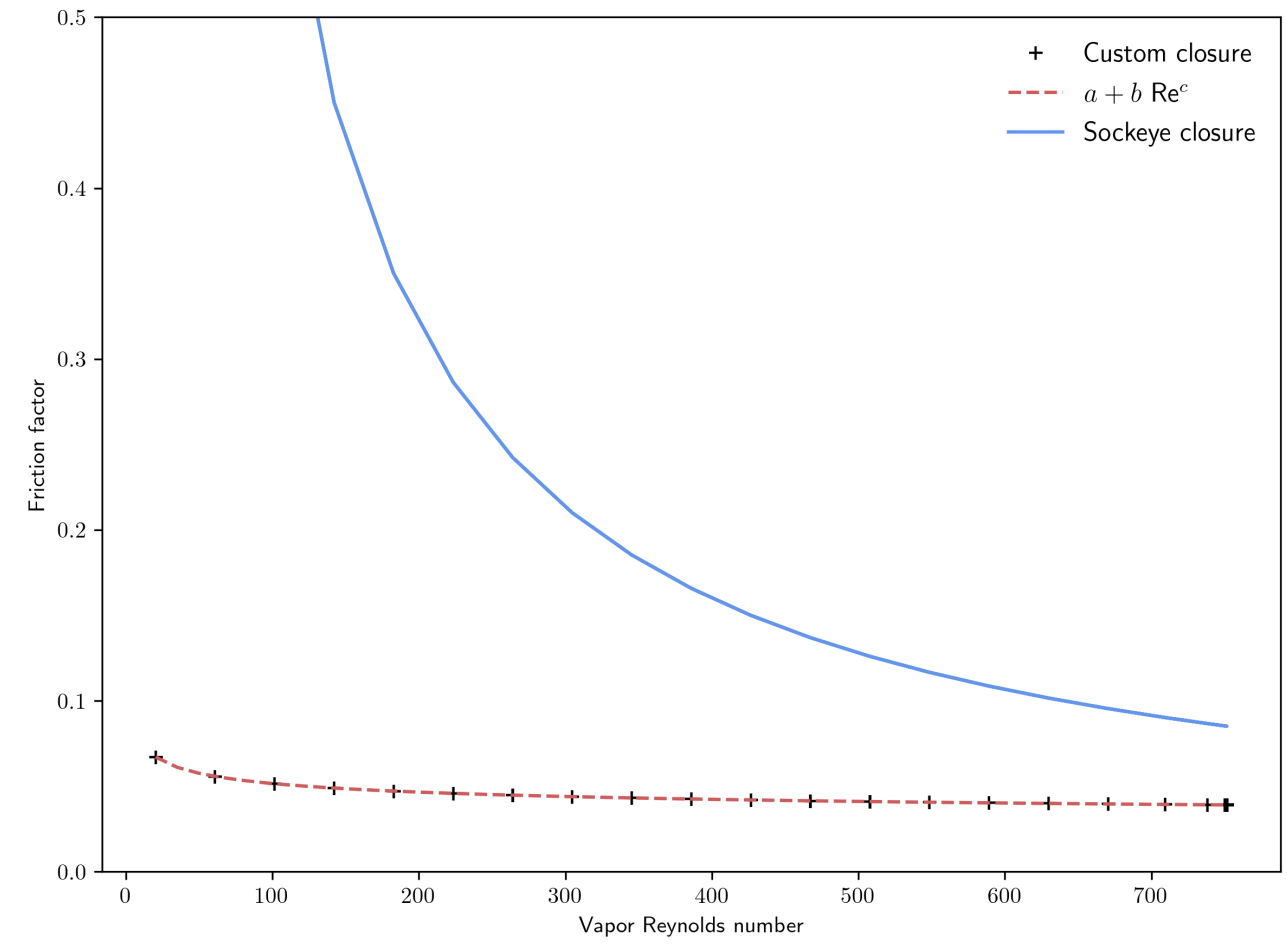

Figure 17: Custom friction factor example.

Figure 18 shows the vapor pressure distributions obtained with the custom closure vs. the default Sockeye 
closures. The overall pressure drop is lower with the custom closures because the custom closure produces smaller friction coefficients, as shown in Figure 17. This custom closure approach allows the analyst to directly change the calculation of important closure parameter and expand the applicability range of Sockeye without code development.

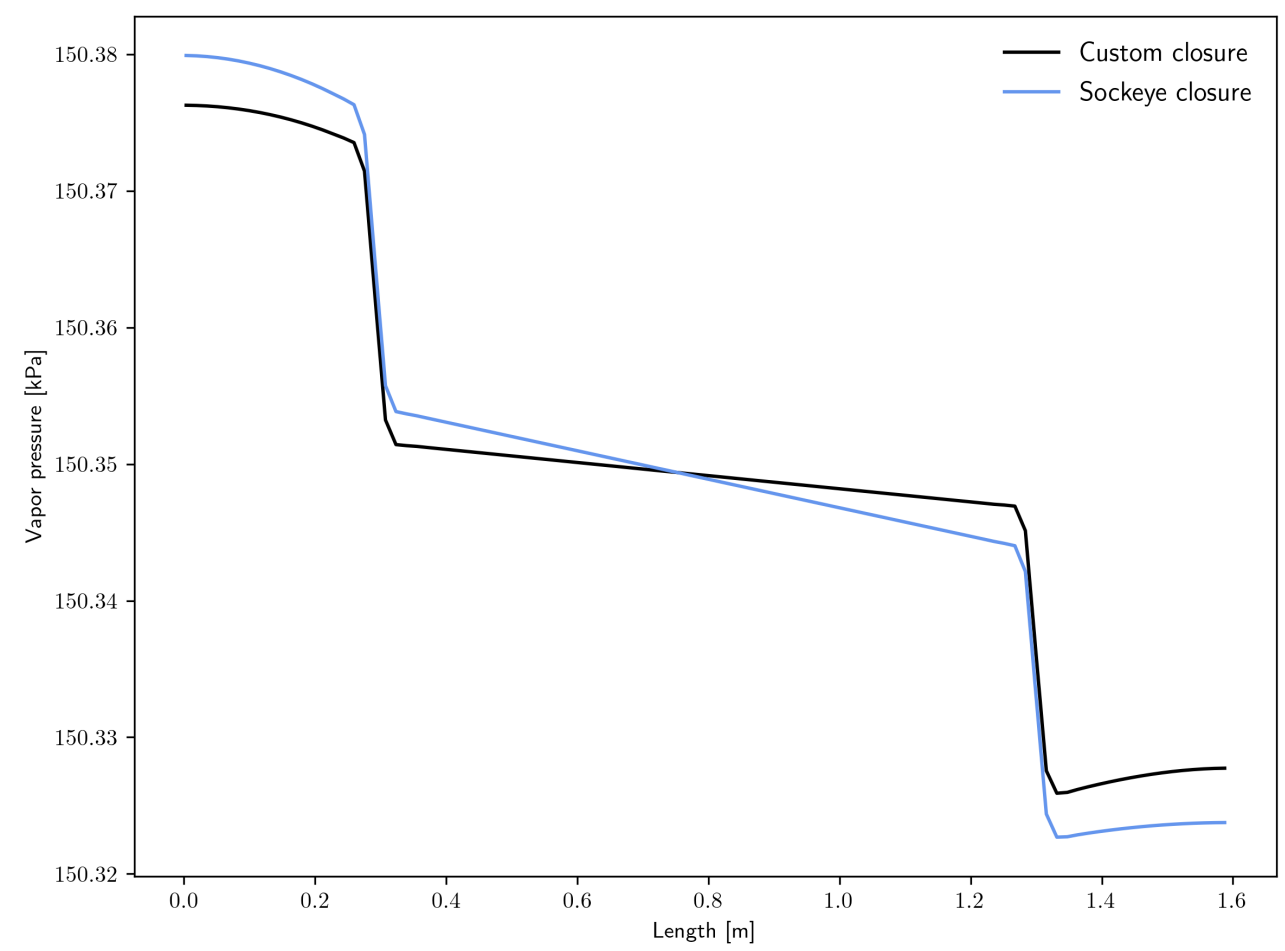

Figure 18: Vapor pressure distribution with custom vapor friction factor.

\subsubsection{Heat Transfer Boundary Conditions}

A heat pipe can exchange heat with its environment through a number of different mechanisms, like convection and radiation, so a user needs to be able to apply these different conditions to accurately simulate the heat transfer rate between the heat pipe and its environment. The use of these heat transfers and boundary conditions are demonstrated in a number of new examples provided in the Sockeye repository, listed in Table 3.

The first set of examples in Table 3 simulate only the 1D working fluid flow and do not couple to 2D heat conduction in the cladding. For this case, two types of heat transfer to the fluid at the heat pipe inner wall are available:

- The heat flux is prescribed: $q=\bar{q}$.

- A convective boundary condition is prescribed: $q=\mathcal{H}\left(T_{\ell}-T_{\text {env }}\right)$, where $T_{\ell}$ is the liquid temperature, $T_{\text {env }}$ is the environment temperature, and $\mathcal{H}$ is the heat transfer coefficient.

The second set of examples in Table 3 simulate the 1D working fluid flow and couple to the 2D heat conduction in the cladding. For this case, several types of boundary conditions can be applied to the outer 
Table 3: List of heat transfer examples.

\begin{tabular}{|c|c|}
\hline File name & Description \\
\hline flow_only.i & 1D flow with a prescribed heat flux \\
\hline flow_only_3phase.i & 1D flow using the 3-phase flow model with a prescribed heat flux \\
\hline flow_only_convection.i & 1D flow with convective heat transfer \\
\hline flow_plus_cladding.i & $\begin{array}{l}\text { 1D flow coupled with } 2 \mathrm{D} \text { heat conduction and a specified heat } \\
\text { flux on the outer wall }\end{array}$ \\
\hline flow_plus_cladding_convection.i & $\begin{array}{l}\text { 1D flow coupled with } 2 \mathrm{D} \text { heat conduction and a convective bound- } \\
\text { ary condition on the outer wall }\end{array}$ \\
\hline flow_plus_cladding_convection_3phase.i & Same as above with the 3 -phase flow model \\
\hline flow_plus_cladding_radiation.i & $\begin{array}{l}\text { 1D flow coupled with } 2 \mathrm{D} \text { heat conduction and radiative boundary } \\
\text { condition on the evaporator outer wall }\end{array}$ \\
\hline
\end{tabular}

wall of the heat pipe:

- The heat flux is prescribed: $q=\bar{q}$.

- A convective boundary condition is prescribed: $q=\mathcal{H}\left(T-T_{\text {env }}\right)$, where $T$ is the cladding outer wall temperature, $T_{\text {env }}$ is the environment temperature, and $\mathcal{H}$ is the heat transfer coefficient.

- A radiative boundary condition is used to define the heat flux: $q=\epsilon \sigma F\left(T^{4}-T_{\mathrm{env}}^{4}\right)$, where $T$ is the cladding outer wall temperature, $T_{\text {env }}$ is the environment temperature, $\sigma$ is the Stefan-Boltzmann constant, $\epsilon$ is the emissivity, and $F$ is a view factor.

\subsubsection{Capillary and Sonic Limits}

The evaluation of the operational limits of a heat pipe is a critical step to define the operating envelope of a given heat pipe design. Some analytic relations have been derived [2], but they rely on a number of assumptions. The flow model implemented in Sockeye can be used to demonstrate these limits, and this work created examples to demonstrate the sonic and capillary limits.

To reproduce a given limit heat rate, we used the following procedure. First, we establish a steady state at low power. Then, the power is slowly increased to stay in a quasi-steady state. Variables of interest are monitored, and the simulation is terminated when the limit is exceeded.

The capillary limit is detected when the capillary pressure reaches its maximum. With the current model, this occurs when the interface between the liquid and vapor phases is fully hemispherical and located at the inner surface of the wick, assuming that the wick is initially fully filled. The vapor volume fraction is monitored, and the simulation terminated when the threshold is exceeded.

Similarly, the sonic limit is detected when the vapor velocity exceeds the speed of sound. To capture the sonic limit, the velocity of the vapor phase is monitored, and the simulation is terminated when it exceeds the speed of sound.

The resulting limits are shown in Figure 19 for the capillary limit. The solid lines are the analytic limits calculated using the utility described in Section 2.5.1. Note that the boiling limit does not appear on this plot because it is much higher than the other limits. The state points for the limit calculation with the flow model have been chosen so that the capillary limit would be the first one encountered. The limit predicted by the flow model follows a similar trend as the analytic one. 
The analytic limit calculation assumes there is no wall friction for the vapor phase and that the wick is perfectly filled. The Sockeye flow model can be used to quantify the effect of these assumptions on the limit calculation. The limit calculation was repeated with a vapor friction factor of 0 . Figure 19 shows that the vapor friction has a negligible effect on the limit. In another set, the same mass of sodium was used for each temperature and corresponded to a perfect fill at the reference temperature of $1000 \mathrm{~K}$. The resulting limits are lower due to the formation of a condenser pool. This shows that the mass of working fluid has a significant impact on the operating range and can be captured by the flow model.

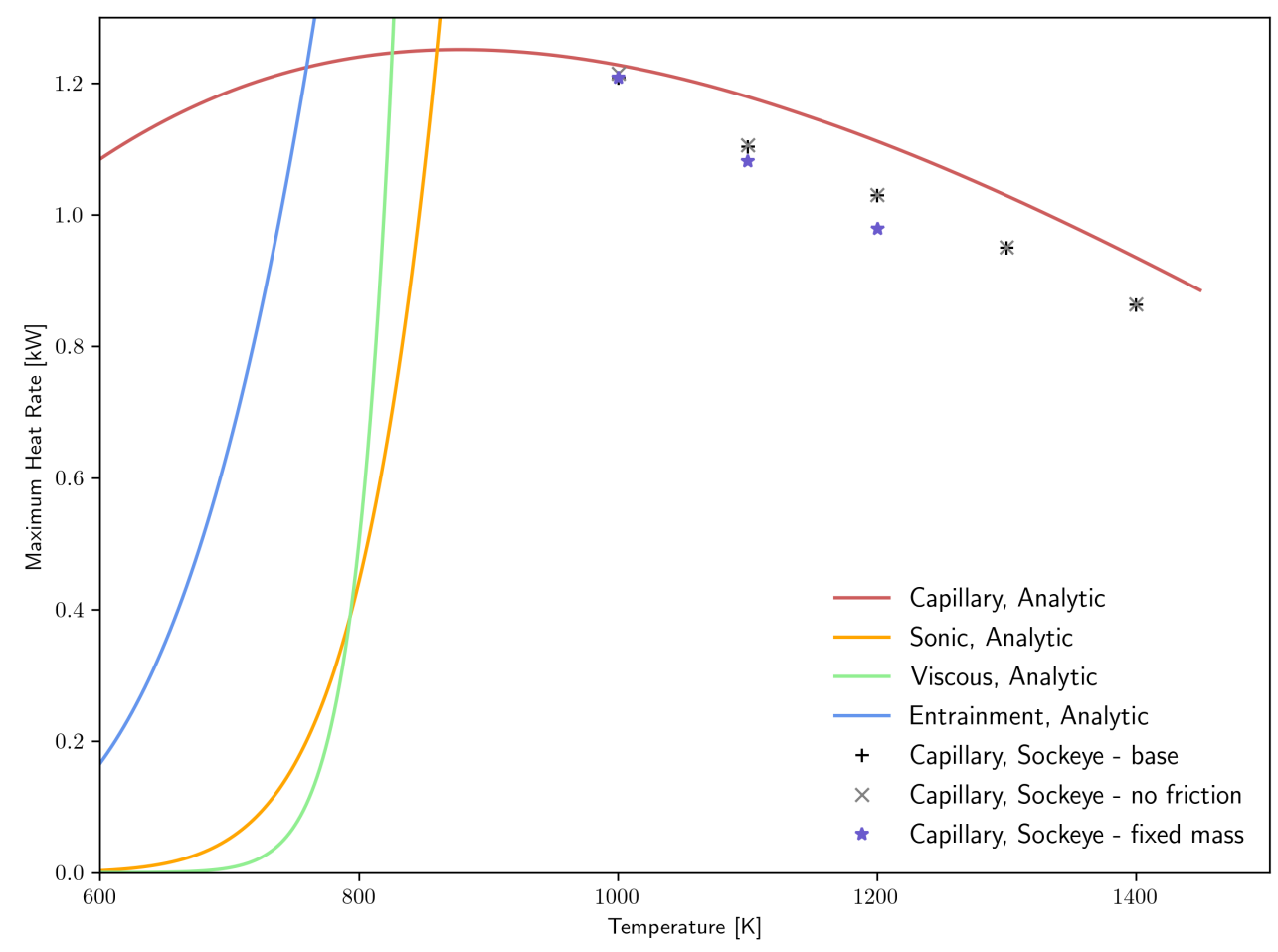

Figure 19: Capillary limit example.

The results for the sonic limit are shown in Figure 20. For these simulations, the permeability was increased so the capillary limit would not interfere with the calculation of the sonic limit and allow a wider range of operating temperature. On the low temperature side, the sonic limit is bounded by the viscous limit. The analytic entrainment limit appears close to the sonic limit, but this limit is not captured by the current flow model and will not cause a failure. There are some differences between the analytic formulation and the flow model, but the values are close and follow the same trend.

\subsection{Discretization Documentation}

Knowledge of the spatial discretization is important because the scheme determines a number of properties of the solution, such as accuracy, spatial convergence rate, and stability. Also, it is necessary for interpreting the solution values output from a code, for example, if they correspond to values at cell edges or cell centers. All of the spatial discretization details are now given in the Sockeye theory manual [17] and will not all be duplicated here, but this report gives a summary and some excerpts. Note that [17] corresponds to a revision that does not contain these changes; a new revision is in the process of being reviewed and 


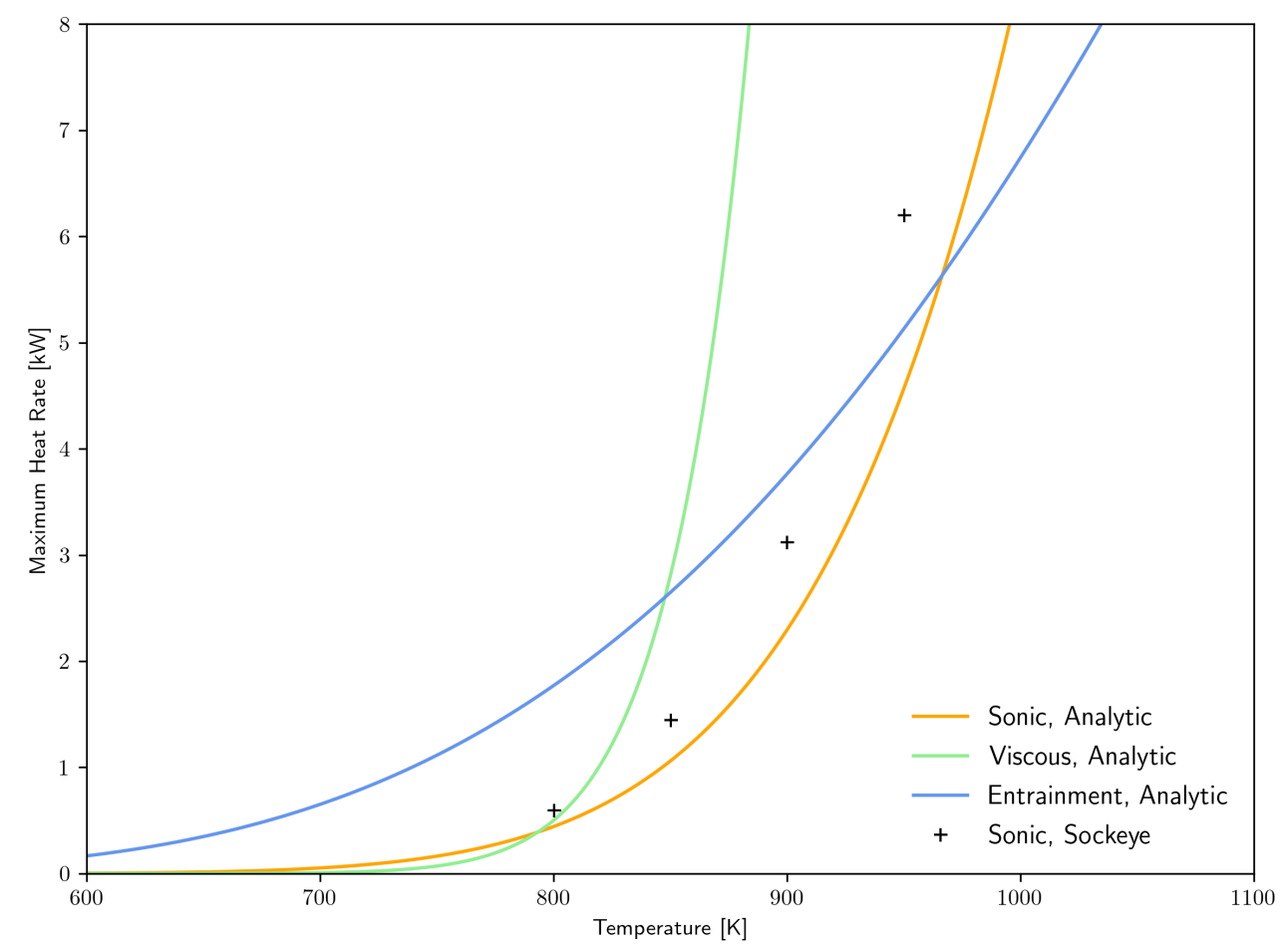

Figure 20: Sonic limit example.

released. Nevertheless, users of Sockeye have access to the latest state of the theory manual, as it is a part of the distributed code repository.

Sockeye is discretized in space using the scheme by Furfaro et al. [29], which is equivalent to a Godunovtype finite volume scheme. The solution thus consists of cell-center values representing averages over the cell and, in the context of the finite element method, is described as having piecewise-constant basis functions. The partial differential equations (PDEs) discretized in Sockeye can be expressed as follows:

$$
\begin{gathered}
\frac{\partial \mathbf{u}_{k}}{\partial t}+\frac{\partial \mathbf{f}_{k}(\mathbf{u})}{\partial x}+\alpha_{k} \frac{\partial \mathbf{h}_{k}(\mathbf{u})}{\partial x}+\frac{\partial \mathbf{m}_{k}(\mathbf{u})}{\partial x}=\mathbf{s}_{k}(\mathbf{u}), \\
\mathbf{u}_{k} \equiv\left[\begin{array}{c}
\alpha_{k} A \\
\alpha_{k} \rho_{k} A \\
\alpha_{k} \rho_{k} u_{k} A \\
\alpha_{k} \rho_{k} E_{k} A
\end{array}\right], \quad \mathbf{u} \equiv\left[\begin{array}{l}
\mathbf{u}_{\ell} \\
\mathbf{u}_{v}
\end{array}\right], \\
\mathbf{f}_{k}(\mathbf{u}) \equiv\left[\begin{array}{c}
\alpha_{k} u_{\text {int }} A \\
\alpha_{k} \rho_{k} u_{k} A \\
\alpha_{k}\left(\rho_{k} u_{k}^{2}+p_{k}\right) A-\alpha_{k} p_{\text {int }} A \\
\left.\rho_{k}\right) A-\alpha_{k} p_{\text {int }} u_{\text {int }} A
\end{array}\right], \quad \mathbf{h}_{k}(\mathbf{u}) \equiv\left[\begin{array}{c}
-u_{\text {int }} A \\
0 \\
0 \\
p_{\text {int }} A \\
p_{\text {int }} u_{\text {int }} A
\end{array}\right], \\
\mathbf{m}_{\ell}(\mathbf{u}) \equiv\left[\begin{array}{c}
0 \\
0 \\
0 \\
0 \\
-\alpha_{\ell} k_{\ell} \frac{\partial T_{\ell}}{\partial x} A
\end{array}\right],
\end{gathered}
$$


and $\mathbf{s}_{k}(\mathbf{u})$ includes a large number of source terms, which are not relevant for this discussion. Note that, in general, heat pipes can be in any orientation in space; the coordinate $x$ here refers to the axial coordinate of the heat pipe. Integrating over each cell volume $\Delta x_{i}$ gives

$$
\begin{aligned}
\left|\Delta x_{i}\right| \frac{\mathrm{d} \mathbf{u}_{k, i}}{\mathrm{~d} t}+\mathbf{f}_{k}\left(\mathbf{u}\left(x_{i+1 / 2}\right)\right)-\mathbf{f}_{k}\left(\mathbf{u}\left(x_{i-1 / 2}\right)\right) & +\alpha_{k, i}\left(\mathbf{h}_{k}\left(\mathbf{u}\left(x_{i+1 / 2}\right)\right)-\mathbf{h}_{k}\left(\mathbf{u}\left(x_{i-1 / 2}\right)\right)\right) \\
& +\mathbf{m}_{k}\left(\mathbf{u}\left(x_{i+1 / 2}\right)\right)-\mathbf{m}_{k}\left(\mathbf{u}\left(x_{i-1 / 2}\right)\right) \approx \int_{\Delta x_{i}} \mathbf{s}_{k}(\mathbf{u}) \mathrm{d} x,
\end{aligned}
$$

where $\mathbf{u}_{k, i}$ denotes the cell-average value:

$$
\mathbf{u}_{k, i}(t) \equiv \frac{1}{\left|\Delta x_{i}\right|} \int_{\Delta x_{i}} \mathbf{u}_{k}(x, t) \mathrm{d} x
$$

The fluxes $\mathbf{f}_{k}\left(\mathbf{u}\left(x_{i+1 / 2}\right)\right), \mathbf{h}_{k}\left(\mathbf{u}\left(x_{i+1 / 2}\right)\right)$, and $\mathbf{m}_{k}\left(\mathbf{u}\left(x_{i+1 / 2}\right)\right)$ are approximated using an approximate Riemann solver. The details of this Riemann solver are summarized briefly here. A characteristics analysis for a hyperbolic system of PDEs can be used to obtain solutions to a Riemann problem, which is defined as a problem having constant left and right states separated by a discontinuity. Approximate Riemann solvers solve this elemental problem approximately, and this forms the basis for many finite volume schemes, where a Riemann problem is solved at each cell interface. The exact solution to a Riemann problem consists of a number of waves separating various solution states. In this case, for a phase $k$, this can be depicted as in Figure 21. The velocity of the liquid-vapor interface is denoted by $u_{\text {int }}$, and various wave speeds are denoted by $S_{k, X}$. A Riemann solver estimates these wave speeds and then uses various conditions, such as the Rankine-Hugoniot condition to solve for the solution states, such as $\mathbf{u}_{k, \mathrm{~L}}^{*}$. Then, the solution is sampled at $t=0$ to determine which state exists at the interface.

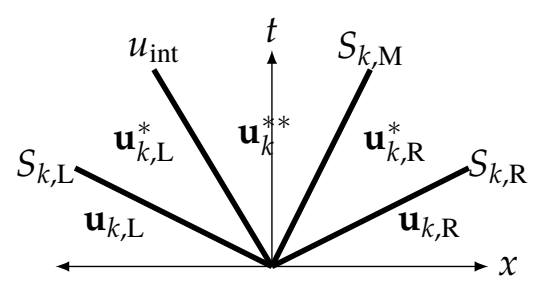

(a) Case $S_{k, \mathrm{~L}}<u_{\text {int }}<S_{k, \mathrm{M}}<S_{k, \mathrm{R}}$.

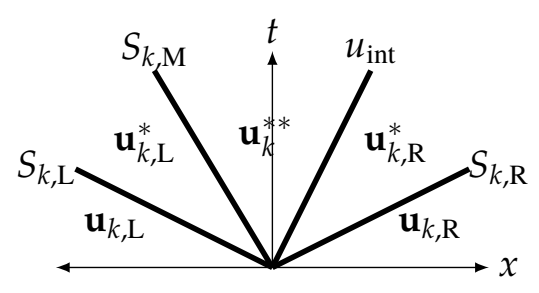

(b) Case $S_{k, \mathrm{~L}}<S_{k, \mathrm{M}}<u_{\text {int }}<S_{k, \mathrm{R}}$.

Figure 21: $x$ - $t$ diagrams for the phase $k$ equations.

The input "left" and "right" states to the Riemann solver can either be the cell-center solution values to either side of the interface or some higher-order estimation of the solution at the interface. For the former case, the scheme is referred to as a Godunov scheme, which is first-order accurate in space. The latter can be accomplished using a number of techniques but, in Sockeye, is accomplished using slope reconstruction. The idea of slope reconstruction is to compute slopes for each cell $i$ of the solution variables, either in the conservative set $\mathbf{u}$ or a primitive set $\mathbf{w}$. Sockeye uses a primitive set. These slopes are computed from the cell-center solution values from a number of adjacent cells and then used to construct a higher-order reconstruction of the solution, which then gets fed into the Riemann solver:

$$
\mathbf{w}_{i}(x, t)=\mathbf{w}_{i}(t)+\left(x-x_{i}\right) \Delta \mathbf{w}_{i},
$$

where $\mathbf{w}_{i}(x, t)$ is the reconstruction, $\mathbf{w}_{i}(t)$ is the cell-average solution, $x_{i}$ is the cell-center position, and $\Delta \mathbf{w}_{i}$ is the slope vector. However, if the slopes are computed without limitation, this can lead to spurious 
oscillations, so a slope limiter is applied. These limiters guarantee certain favorable properties, leading to a total variation diminishing scheme, which ensures the monotonicity of the solution. One well-known slope limiter is the minmod limiter:

$$
\Delta w_{i}=\operatorname{minmod}\left(\Delta w_{i-1 / 2}, \Delta w_{i+1 / 2}\right), \quad \Delta w_{i+1 / 2} \equiv \frac{w_{i+1}-w_{i}}{x_{i+1}-x_{i}},
$$

where $w$ is a component of $\mathbf{w}$. Other slope limiters, also documented in the theory manual, include monotonized central difference and superbee. For smooth problems, second-order accuracy in space is expected when using slope reconstruction, even when using these limiters.

\section{VERIFICATION AND VALIDATION}

This section discusses some verification and validation assessments that were performed in response to customer request for verification and validation activities. These assessments appear in [30]. This section is organized as follows: Section 5.1 describes some comparisons made to analytic solutions, Section 5.2 describes a verification of spatial convergence rates, and Section 5.3 describes a comparison to experimental data for the SAFE-30 heat pipe module test.

\subsection{Analytic Solutions}

In this section, a test problem is described where analytic solutions were derived from the governing partial differential equations used by Sockeye. Two subproblems were developed for demonstrations of these solutions. The first uses a "single-ended" heat pipe configuration, which corresponds to that illustrated in Figure 1b; that is, there is a single evaporator region and a single condenser region. The second subproblem uses a "double-ended" configuration, where, instead of a single condenser region, there are two condenser regions on opposite ends of the heat pipe. The single-ended and double-ended configurations are illustrated in Figures 22a and 22b, respectively.

Note in each case, the heat pipe used is exactly the same; that is, there are no manufacturing differences, only usage differences. Some commonly employed heat pipe analyses fail to consider configurations other than the standard single-ended configuration, but Sockeye's flow models make no assumption in this regard and thus retain full flexibility.

Table 4 summarizes the heat pipe parameters and conditions used for this test problem. The input thermal power is imposed directly and uniformly over the evaporator and equally removed from the condenser region(s). For the single-ended configuration, the evaporator is along the first $0.2 \mathrm{~m}$, followed by a $0.4-\mathrm{m}$ adiabatic section, and then a $0.4-\mathrm{m}$ condenser. For the double-ended configuration, the evaporator section is the middle $0.2 \mathrm{~m}$, and the condenser is split into two 0.2-m segments, one on each end, with 0.2-m adiabatic sections dividing evaporator and condenser sections, making a symmetric problem about the axial midpoint.

Sockeye results include the volume fraction, thermodynamic state, and axial velocity for each phase. Where possible, results are compared with analytic solutions.

Sockeye's governing partial differential equations are given by Equation (61). Recall that $x$ refers to the axial coordinate of the heat pipe. Defining the mass flow rate of a phase $k$ as $\dot{m}_{k} \equiv \alpha_{k} \rho_{k} u_{k} A$, the conservation of mass equations become the following for steady conditions:

$$
\frac{\mathrm{d} \dot{m}_{\ell}}{\mathrm{d} x}=-\Gamma_{\mathrm{int}} a_{\mathrm{int}} A, \quad \frac{\mathrm{d} \dot{m}_{v}}{\mathrm{~d} x}=\Gamma_{\mathrm{int}} a_{\mathrm{int}} A
$$




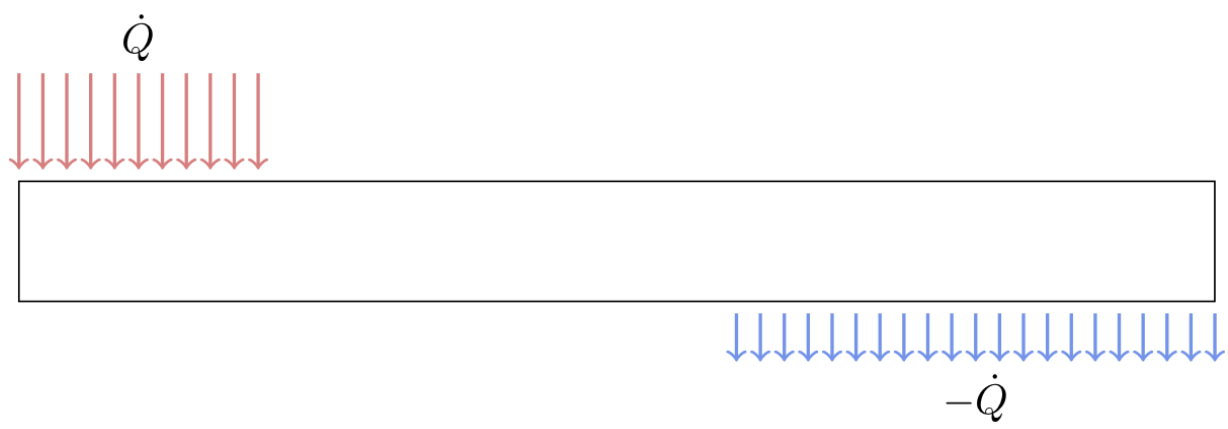

(a) Single-ended configuration.

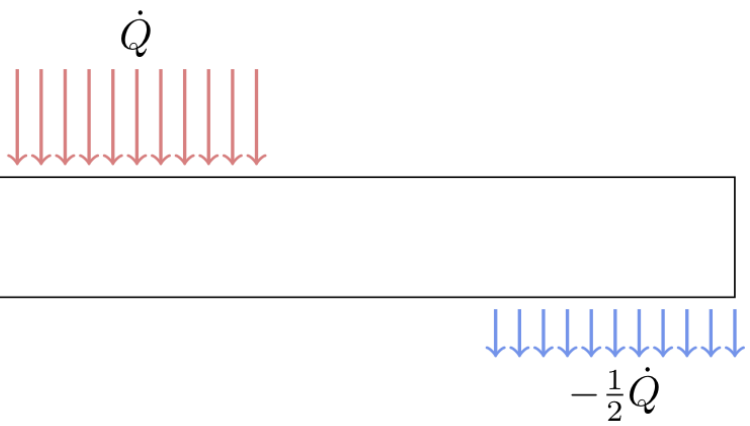

(b) Double-ended configuration.

Figure 22: Configurations for analytic solutions comparisons.

Then, the following assumptions are employed for the conservation of energy equations:

- Steady

- Negligible energy change due to body forces (e.g., friction and gravity)

- Negligible axial heat conduction

- Negligible energy flux due to pressure (i.e., $\frac{\mathrm{d} \alpha_{k} u_{k} p_{k} A}{\mathrm{~d} x} \approx 0$ )

- Negligible total energy gradient (i.e., $\frac{\mathrm{d} E_{x}}{\mathrm{~d} x} \approx 0$ ).

Substituting Equation (66) into the energy equations resulting from the above assumptions and then summing them gives

$$
\frac{\mathrm{d} \dot{m}_{v}}{\mathrm{~d} x}=-\frac{\mathrm{d} \dot{m}_{\ell}}{\mathrm{d} x}=\frac{q^{\prime}(x)}{h_{\ell v}},
$$

where $q^{\prime}=q_{\text {wall }} P_{\text {wall }}$ is the linear wall heat rate and $h_{\ell v}$ is the latent heat of vaporization. Now, to get the axial velocity profile, the following additional assumptions are made:

- Density and latent heat of vaporization are uniform

- The wick is perfectly saturated: $\alpha_{v}=\alpha_{v}^{\text {wick,i, }, 0}$. 
Table 4: Heat pipe specifications for analytic solutions comparisons.

\begin{tabular}{lr}
\hline Parameter & Value \\
\hline Working fluid & Sodium \\
Wick inner diameter, $D_{\text {wick,i }}$ & $0.01231 \mathrm{~m}$ \\
Wick outer diameter, $D_{\text {wick,o }}$ & $0.01259 \mathrm{~m}$ \\
Cladding inner diameter, $D_{\text {clad,i }}$ & $0.01410 \mathrm{~m}$ \\
Heat pipe length, $L_{\mathrm{hp}}$ & $1.0 \mathrm{~m}$ \\
Wick porosity, $\epsilon$ & 0.770621 \\
Pore radius, $R_{\text {pore }}$ & $23 \times 10^{-6} \mathrm{~m}$ \\
Wick permeability, $K$ & $10^{-10} \mathrm{~m}^{2}$ \\
\hline Orientation & Horizontal \\
Initial temperature, $T_{\ell}, T_{v}$ & $1200 \mathrm{~K}$ \\
Initial working fluid volume & $1.01\left(1-\alpha_{v}^{\text {wick, }, 00}\right) A L_{\mathrm{hp}}$ \\
Power, $Q$ & $1 \mathrm{~kW}$ \\
\hline
\end{tabular}

With these assumptions,

$$
u_{v}(x)=\frac{\int_{0}^{x} q^{\prime}\left(x^{\prime}\right) \mathrm{d} x^{\prime}}{\rho_{v} h_{\ell v} A_{v}}, \quad u_{\ell}(x)=-\frac{\int_{0}^{x} q^{\prime}\left(x^{\prime}\right) \mathrm{d} x^{\prime}}{\rho_{\ell} h_{\ell v} A_{\ell}}
$$

where $A_{v}=\frac{\pi}{4} D_{\text {wick, } \mathrm{i}}^{2}$ is the vapor flow cross-sectional area and $A_{\ell}=A-A_{v}$ is the liquid flow crosssectional area.

For these demonstrations, 100 uniform elements are used along the length, and BDF2 time integration with an adaptive time step size is used and run until steady. Velocities for both the single-ended and doubleended configurations are shown in Figure 23 and compared with the analytical solutions given in Equation (68), showing excellent agreement. With uniform heating and cooling, the velocities change linearly, in accordance with Equation (68). For the double-ended configuration, the total mass flow rate required for the given power transmission is split between two ends; thus the maximum speed of either phase is cut by a factor of 2. The decreased average speed of the double-ended configuration implies a lower pressure drop, since frictional terms in both phases are proportional to speed.

Since equal power is added and removed, there is no net energy addition into the system, and thus the steady solution should be a redistribution of the initial energy of the system at the initial, uniform temperature of $1200 \mathrm{~K}$. This, combined with the observation of the very small temperature difference that heat pipes typically operate on, indicates that the final temperature distribution should show a profile that is nearly uniform and around $1200 \mathrm{~K}$. Figure 24 shows the liquid and vapor temperature profiles for both configurations and indicates that the results are consistent with expectation. For both cases, the temperatures vary less than $3 \mathrm{~K}$ over the length of the heat pipe, around $1200 \mathrm{~K}$. Since heating and cooling are applied to the liquid phase, the liquid phase temperature exceeds the vapor phase in the evaporator and is lower than the vapor phase in the condenser.

The void fraction profile is shown in Figure 25. The value $\alpha_{v}^{\text {wick,i,0 }}$ corresponds to the liquid-vapor interface flat at the inner surface of the wick, and $\alpha_{v}^{\text {wick,i, }+}$ corresponds to a full curvature inward at the inner surface of the wick. Therefore, for both configurations, the liquid-vapor interface is only slightly curved for most of the heat pipe length with some minor pooling of the liquid at the condenser end(s). This pooling is 


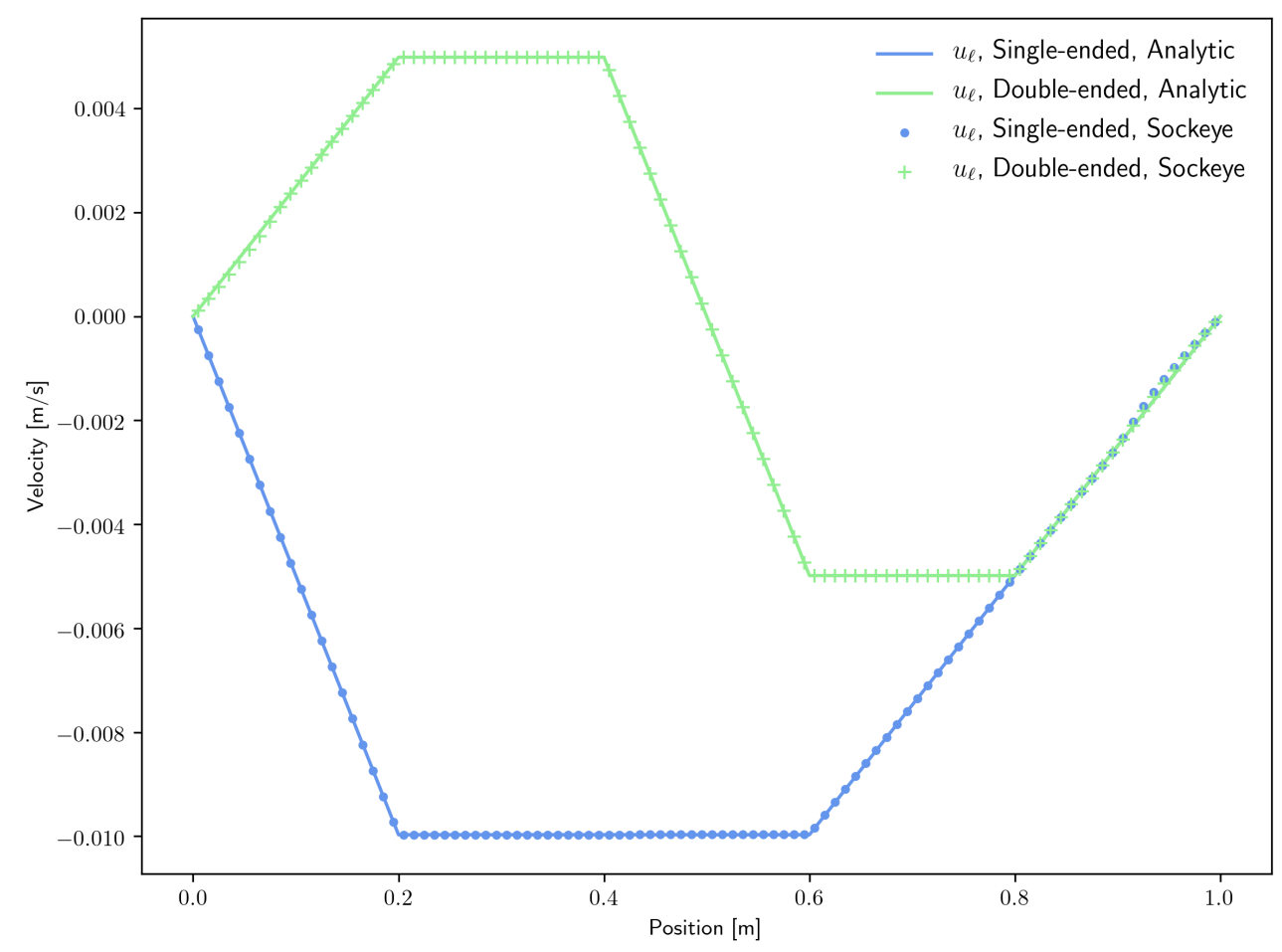

(a) Comparison to analytic liquid velocity solutions.

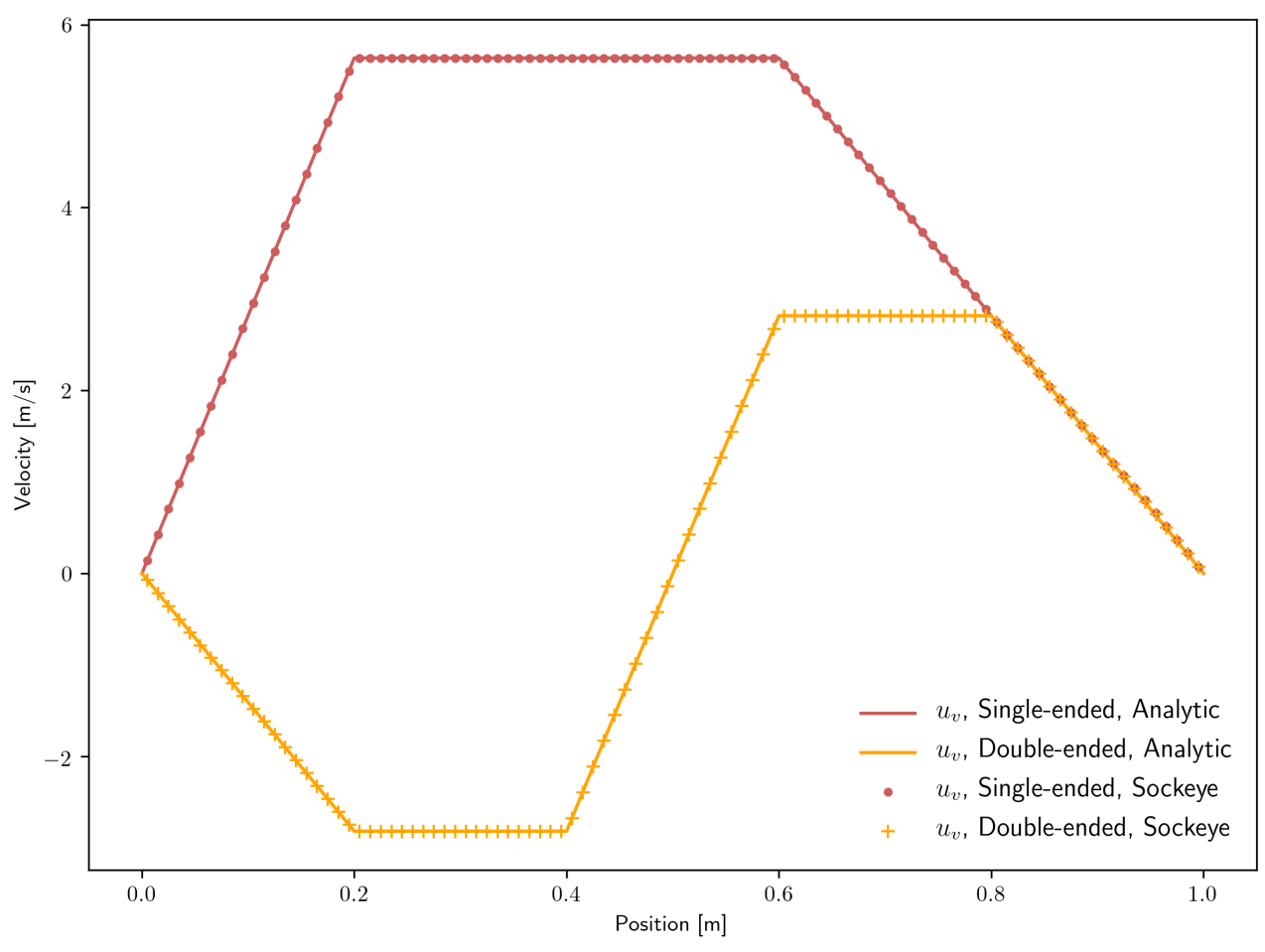

(b) Comparison to analytic vapor velocity solutions.

Figure 23: Comparison to analytic velocity solutions. 


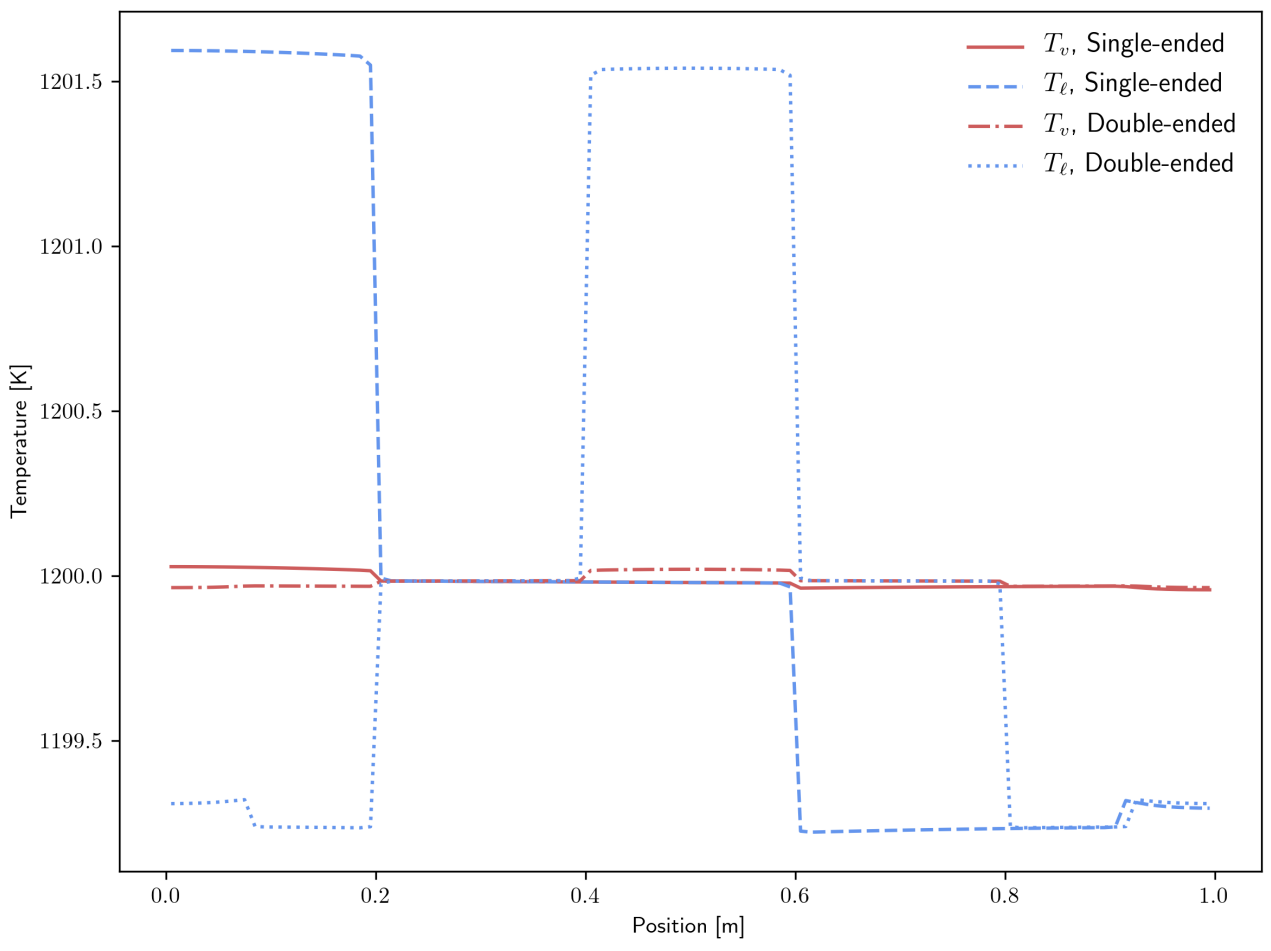

Figure 24: Steady temperature solutions for single-ended vs. double-ended configurations.

the result of the $1 \%$ excess working fluid volume, as indicated by Table 4 .

The pressure distributions of both phases are shown for both configurations in Figure 26. For both configurations, the liquid pressure drop is dominant to the vapor pressure drop. Wet points (where liquid and vapor pressure are approximately equal) appear at the end of the condenser section(s). In the case of the double-ended configuration, the liquid and vapor flow paths are decreased by a factor of 2 over the single-ended configuration, thus leading to significantly smaller pressure drops.

Pressure solutions are compared against analytical pressure drop relations for the single-ended configuration. For the vapor phase, Cotter's theory [31] is used. The radial Reynolds number for the vapor phase,

$$
\operatorname{Re}_{r, v} \equiv \frac{\rho_{v} w_{v} R_{v}}{\mu_{v}}=\frac{1}{2 \pi \mu_{v}} \frac{\mathrm{d} \dot{m}_{v}}{\mathrm{~d} x}
$$

where $w_{v}$ is the radial vapor velocity component and $R_{v}=\frac{1}{2} D_{\text {wick,i }}$ is used to distinguish between pressure drop formulations. The maximum radial Reynolds number for this test problem was determined to be approximately 11.7, which Cotter's theory classifies as "turbulent," leading to the following pressure drop [2]:

$$
\begin{gathered}
\frac{\mathrm{d} p_{v}}{\mathrm{~d} x}=-\frac{s(x) \dot{m}_{v}(x)}{4 \rho_{v} R_{v}^{4}} \frac{\mathrm{d} \dot{m}_{v}}{\mathrm{~d} x}-\frac{8 a(x) \mu_{v} \dot{m}_{v}(x)}{\rho_{v} \pi R_{v}^{4}}, \\
s(x)= \begin{cases}1 & q(x)>0 \\
0 & q(x)=0 \\
\frac{4}{\pi^{2}} & q(x)<0\end{cases}
\end{gathered}
$$




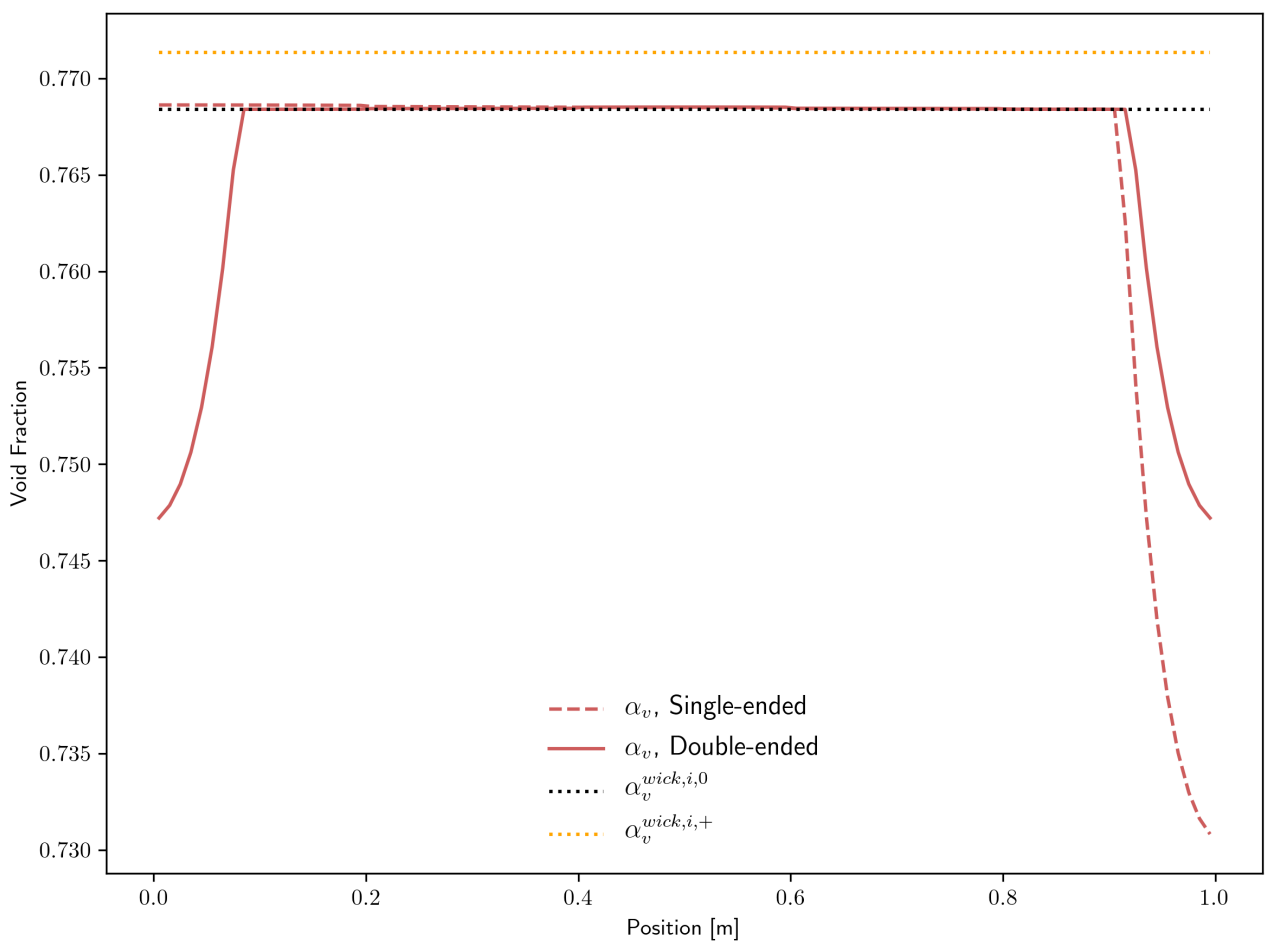

Figure 25: Steady void fraction solutions for single-ended vs. double-ended configurations.

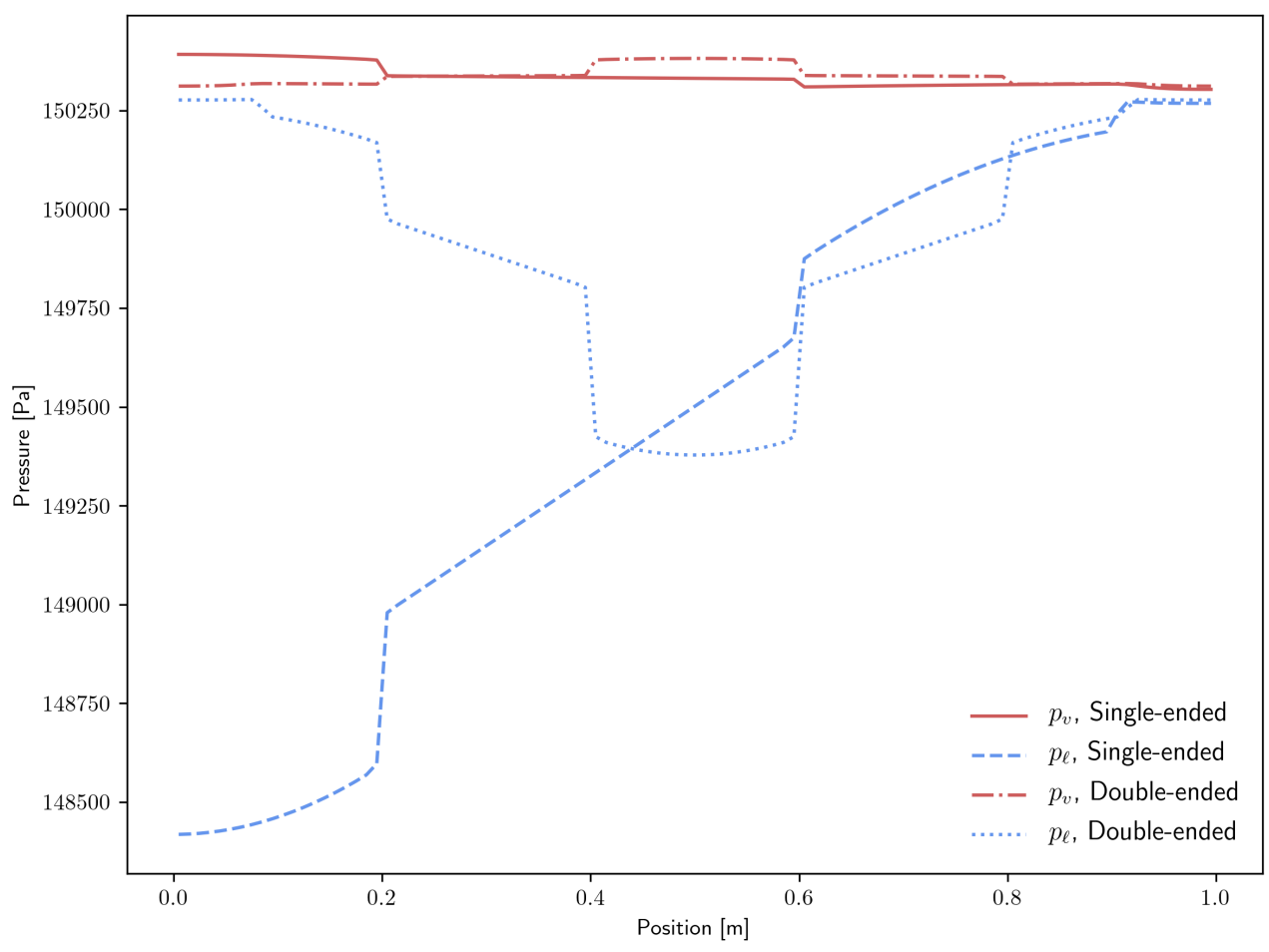

Figure 26: Steady pressure solutions for single-ended vs. double-ended configurations. 


$$
a(x)=\left\{\begin{array}{ll}
1 & q(x)=0 \\
0 & q(x) \neq 0
\end{array} .\right.
$$

Figure 27 shows the comparison of this analytic solution with the pressure solution from Sockeye, as well as the saturation pressure at the vapor temperature. Vapor pressure drops within each region (evaporator, adiabatic, and condenser) match analytical solutions very well. However, Sockeye results indicate pressure drops between these regions that are not shown by the analytic models. Note from the pressure scale that these jumps are actually quite small, on the order of $10 \mathrm{~Pa}$. As for the cause of these jumps, there is strong agreement between Sockeye's pressure curve and saturation pressure curve, which indicates that the vapor phase is nearly following the saturation condition. The saturation pressure profile suggests the reason for these pressure drops: the temperature changes sharply at these locations. The analytic solution does not consider the non-isothermal effects.

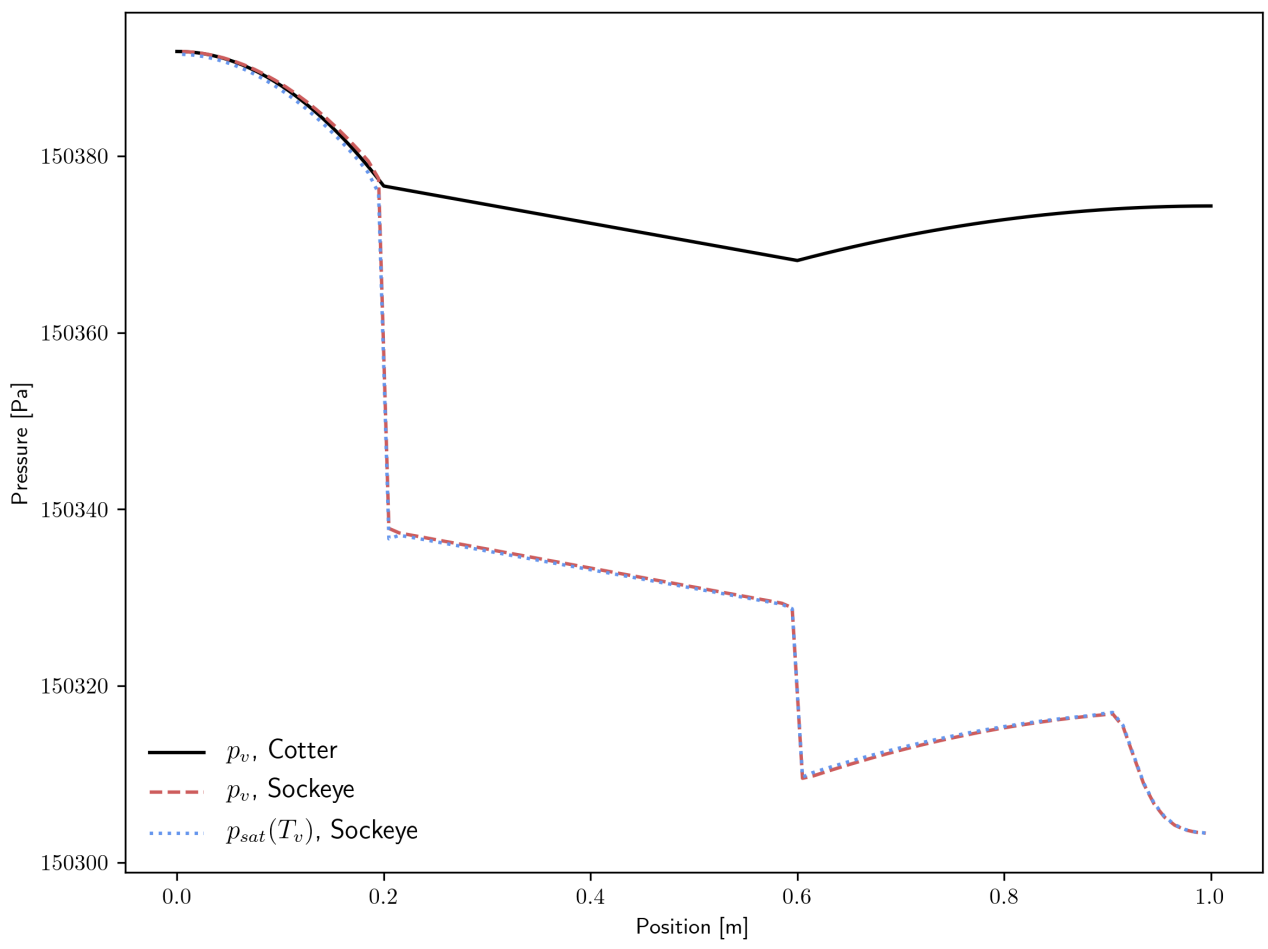

Figure 27: Comparison of vapor pressure drop with analytic solution for single-ended configuration.

For the liquid phase, Darcy's law is used to analyze the pressure drop [2]:

$$
\frac{\mathrm{d} p_{\ell}}{\mathrm{d} x}=-\frac{\mu_{\ell} u_{\ell}}{K} \frac{A_{\text {wick, } \ell}}{\alpha_{\ell} A}
$$

where the factor $A_{\text {wick, } \ell} /\left(\alpha_{\ell} A\right)$ is applied because only a fraction of the liquid channel is through the porous structure. Again, pressure drops within each region match very well to the analytic solution, but pressure drops between regions are significant. Unlike the vapor phase, the liquid phase does not follow the saturation curve.

In summary, these comparisons show excellent agreement with analytic solutions. Velocity profiles are visually indistinguishable from analytic solutions, and pressure drops show excellent agreement within each axial region of the heat pipe, with pressure drops between regions not present in analytic solutions. The 


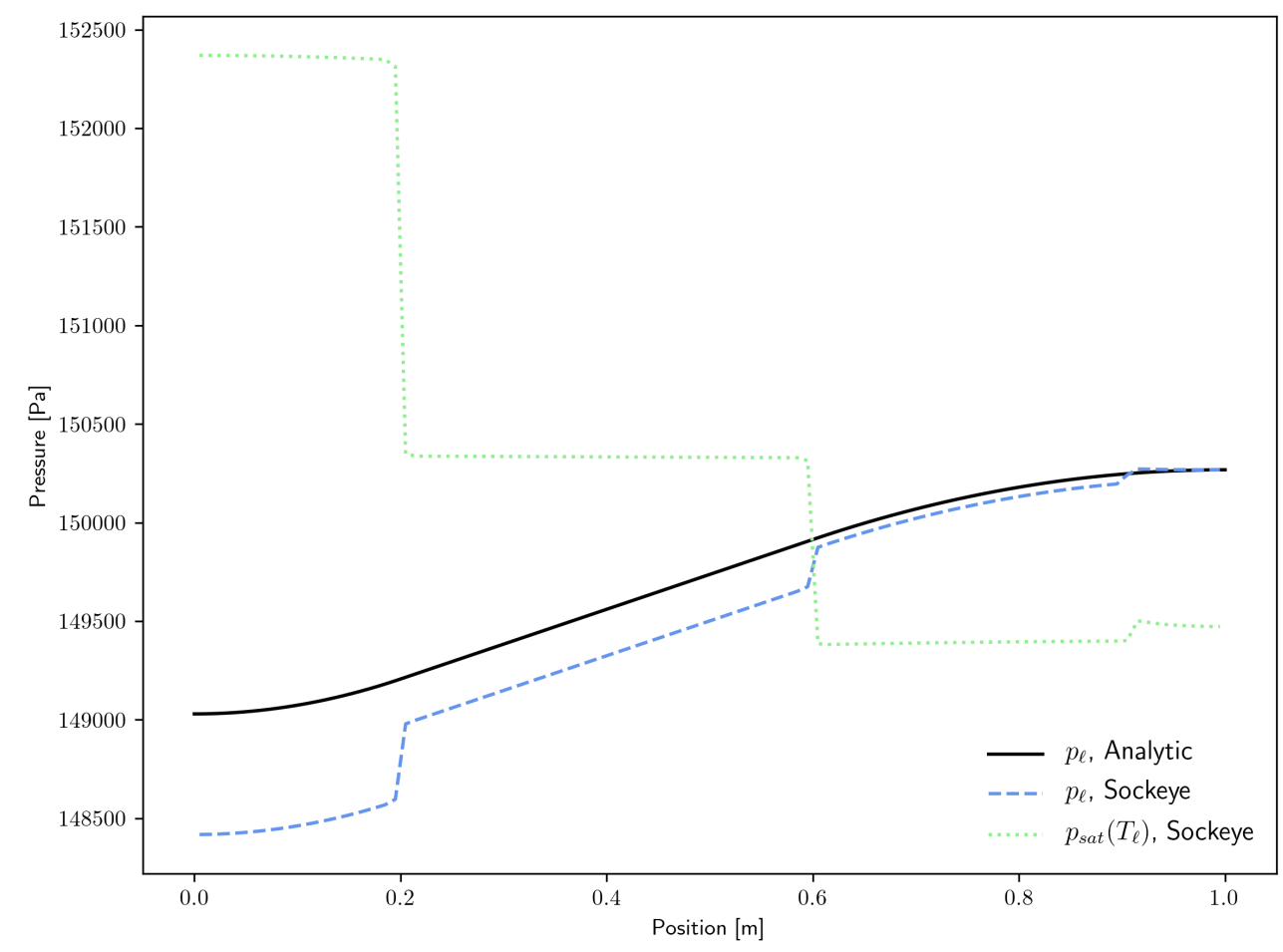

Figure 28: Comparison of liquid pressure drop with analytic solution for single-ended configuration.

vapor pressure drops were shown to be justified by examining the saturation pressure corresponding to the vapor temperature, which indicates a shortcoming of the analytic solutions and conversely a major advantage of numerical simulation. These results are an excellent step toward the code verification that customers require.

\subsection{Spatial Convergence Rate Verification}

This section describes a verification of the spatial convergence order of the flow model used in Sockeye. This verification is essential for demonstrating that the implementation of the spatial discretization, as described in Section 4.2, is correct. To execute this verification, the method of manufactured solutions (MMS) was used. MMS is a very useful technique for verification where the analyst chooses the solution to the problem first. This solution is then substituted into the governing equations to derive source terms. These derived source terms are then implemented in the test problem, and the test verifies that the resulting solution matches the original, chosen solution.

Since the objective here is to verify the spatial convergence order, only spatial operators are important. Therefore, source terms in the equations can be safely dropped without detriment to the analysis, allowing a tractable MMS source derivation. In light of this observation and to simplify analyses by decoupling the phases, the following assumptions are made:

- Constant volume fraction

- Zero interfacial heat transfer coefficients 
- Zero pressure relaxation

- Zero heat conduction

- Infinite permeability

- Zero gravity

- Zero wall friction

- Zero wall heat.

With the first three assumptions, the liquid and vapor phases are effectively decoupled, and with the remaining assumptions, the flow equations reduce to a homogeneous system, to which MMS sources are added, resulting in the following system:

$$
\begin{gathered}
\frac{\partial \alpha_{\ell} \rho_{\ell} A}{\partial t}+\frac{\partial \alpha_{\ell} \rho_{\ell} u_{\ell} A}{\partial x}=S_{\alpha_{\ell} \rho_{\ell} A}, \\
\frac{\partial \alpha_{\ell} \rho_{\ell} u_{\ell} A}{\partial t}+\frac{\partial \alpha_{\ell}\left(\rho_{\ell} u_{\ell}^{2}+p_{\ell}\right) A}{\partial x}=S_{\alpha_{\ell} \rho_{\ell} u_{\ell} A} \\
\frac{\partial \alpha_{\ell} \rho_{\ell} E_{\ell} A}{\partial t}+\frac{\partial \alpha_{\ell} u_{\ell}\left(\rho_{\ell} E_{\ell}+p_{\ell}\right) A}{\partial x}=S_{\alpha_{\ell} \rho_{\ell} E_{\ell} A} \\
\frac{\partial \alpha_{v} \rho_{v} A}{\partial t}+\frac{\partial \alpha_{v} \rho_{v} u_{v} A}{\partial x}=S_{\alpha_{v} \rho_{v} A}, \\
\frac{\partial \alpha_{v} \rho_{v} u_{v} A}{\partial t}+\frac{\partial \alpha_{v}\left(\rho_{v} u_{v}^{2}+p_{v}\right) A}{\partial x}=S_{\alpha_{v} \rho_{v} u_{v} A}, \\
\frac{\partial \alpha_{v} \rho_{v} E_{v} A}{\partial t}+\frac{\partial \alpha_{v} u_{v}\left(\rho_{v} E_{v}+p_{v}\right) A}{\partial x}=S_{\alpha_{v} \rho_{v} E_{v} A}
\end{gathered}
$$

where $S_{i}$ represents the MMS source rate for $i$.

To facilitate the MMS source derivation, the ideal gas equation of state is used for both phases, with different parameters:

$$
p_{k}(\rho, e)=\rho e\left(\gamma_{k}-1\right) \text {, }
$$

where $\gamma_{\ell}=2$ and $\gamma_{v}=1.4$.

The chosen MMS solutions are the following:

$$
\begin{gathered}
\alpha_{k}(x, t)=0.5, \\
\rho_{k}(x, t)=A_{k}\left(\sin \left(B_{k} x+C_{k} t\right)+2\right), \\
u_{k}(x, t)=A_{k} t \sin (\pi x), \\
p_{k}(x, t)=A_{k}\left(\cos \left(B_{k} x+C_{k} t\right)+2\right),
\end{gathered}
$$

where $A_{k}, B_{k}$, and $C_{k}$ are chosen constants for phase $k$. The chosen constants were $A_{\ell}=B_{\ell}=C_{\ell}=1$ and $A_{v}=B_{v}=C_{v}=0.5$.

Runs were performed on the domain $x \in(0,1)$ with a cross-sectional area of $A=1$. To prevent the temporal error from obscuring the spatial error, a small time step size of $\Delta t=10^{-6} \mathrm{~s}$ was used with the 3rd-order, explicit, strong-stability-preserving temporal integration scheme [32]. 10 time steps were used in each of the five runs of different mesh sizes, with the coarsest mesh size being $0.1 \mathrm{~m}$ and refining the mesh 
by a factor of 2 for each subsequent run. Error was computed for density, velocity, and pressure in each phase. The following error norm was used to assess convergence for a quantity $y$ :

$$
\left\|y-y_{h}\right\|_{1} \equiv \sum_{i} h_{i}\left|y\left(x_{i}\right)-Y_{i}\right|
$$

where in this context, $h$ represents the mesh size (i.e., $\Delta x$ ), $y_{h}$ represents the approximate solution of the exact solution $y, i$ is an element index, and $Y_{i}$ is the associated solution value.

Without slope reconstruction, the spatial discretization is described as a Godunov scheme, which is firstorder accurate, and, with a full slope reconstruction, second-order accuracy is expected [33, 34]. Figures 29 and 30 show the results of this study for the Godunov scheme and full-reconstruction scheme, respectively. Reference slopes for first-order and second-order accuracy are provided where relevant. The Godunov scheme demonstrates the expected first-order accuracy for density and pressure, but velocity encounters a second-order accuracy superconvergence for the chosen test problem and error norm. The full slope reconstruction scheme demonstrates universal second-order accuracy in the chosen error norm.

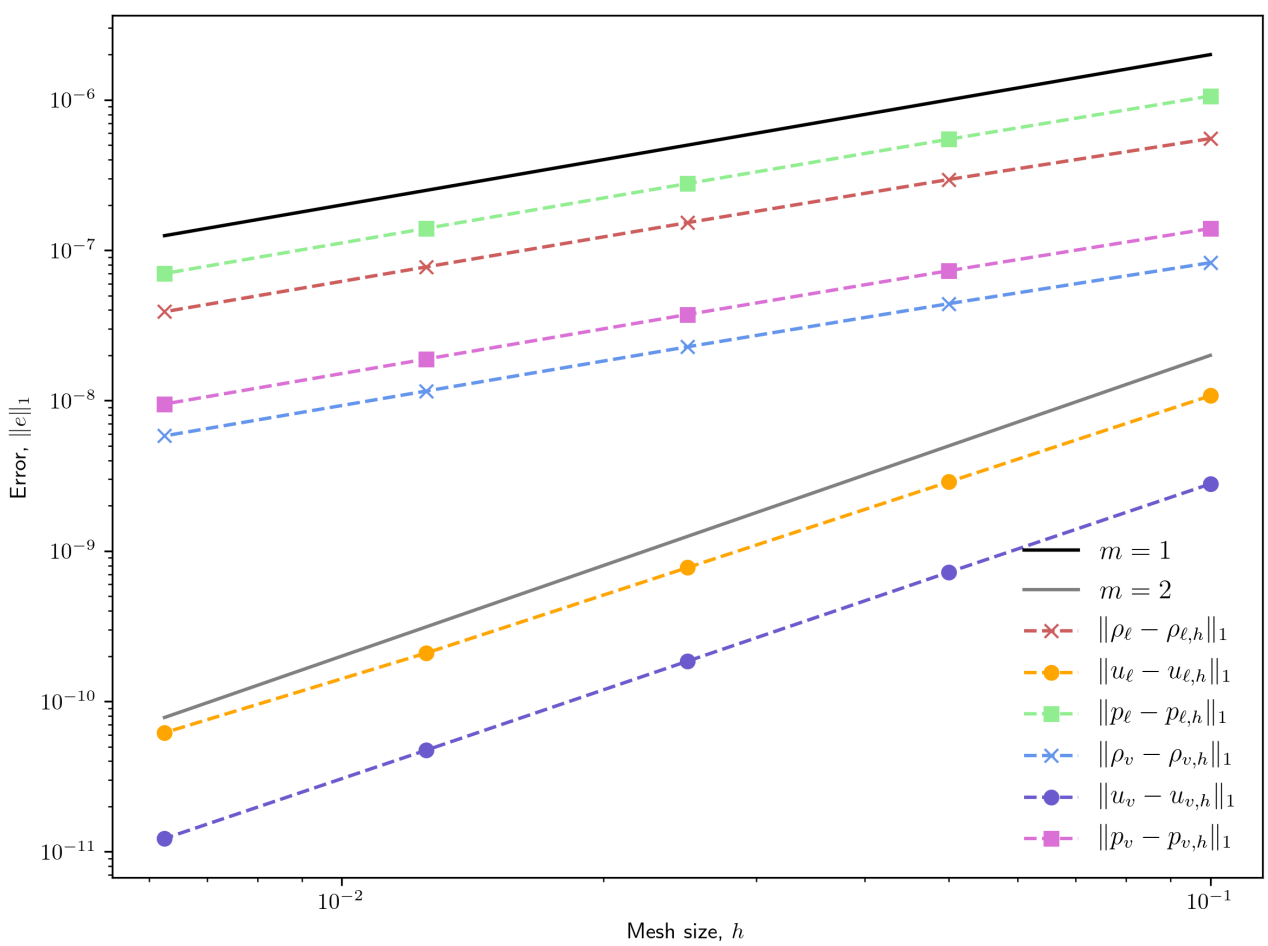

Figure 29: Spatial convergence for the Godunov scheme.

\subsection{SAFE-30 Heat Pipe Module Test}

For this test problem, the SAFE-30 heat pipe module experiment [35] is modeled and compared to experimental data provided by Los Alamos National Laboratory. For this simulation, the 1D, two-phase flow channel is coupled to heat conduction in the heat pipe cladding, which demonstrates the ability to account for the thermal resistance of the heat pipe and the transient effects of the cladding's thermal capacity. This experiment started from room temperature, so the sodium working fluid was initially frozen, but Sockeye is 


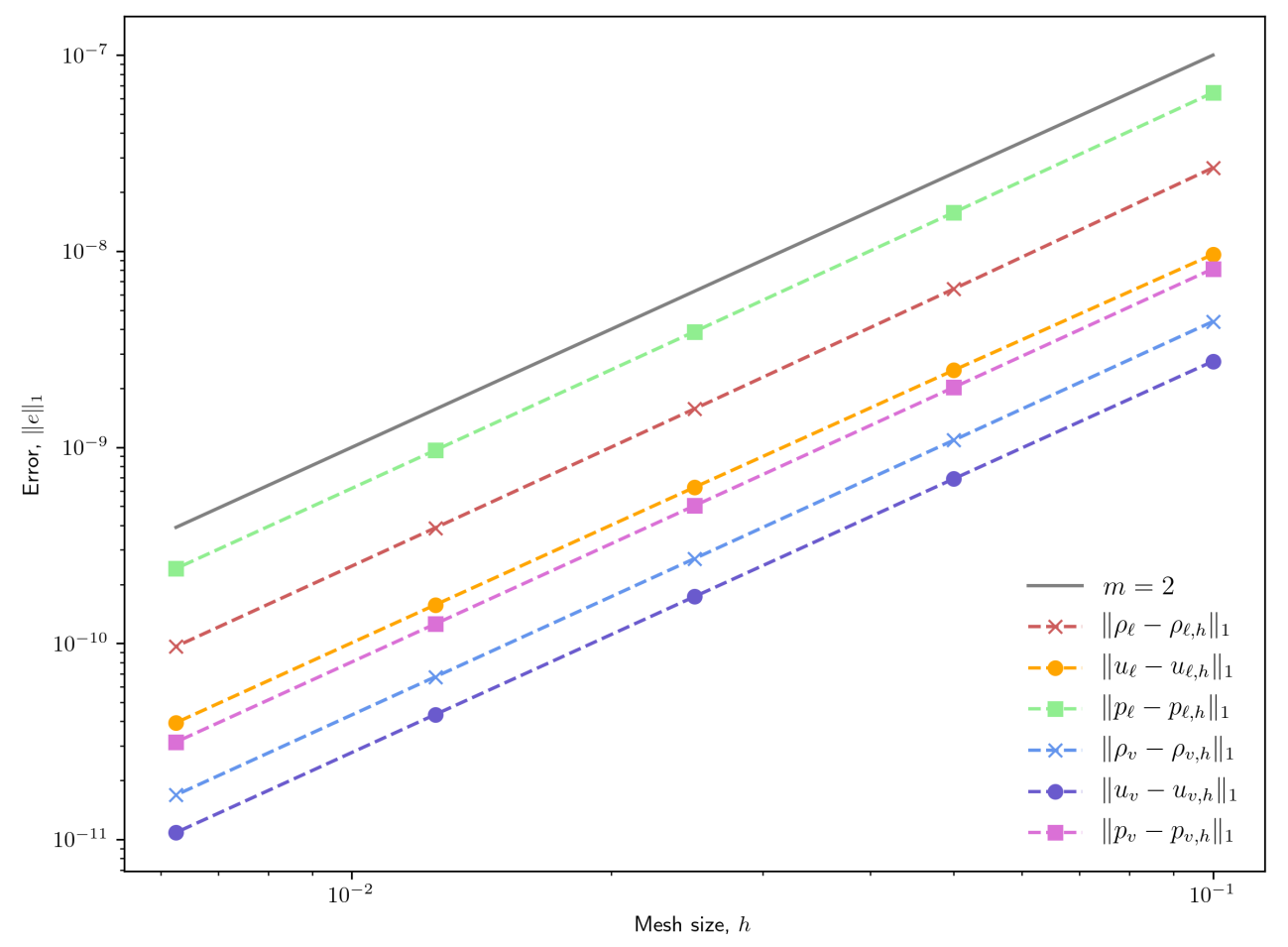

Figure 30: Spatial convergence for the full-reconstruction scheme.

not yet equipped to model startup, so the simulation was started at $t=8,000 \mathrm{~s}$. The experiment was run until $t=25,200 \mathrm{~s}$ (7 hours), but the Sockeye simulation results shown here are at $t=9,575 \mathrm{~s}$ because failure occurs shortly afterward; this failure is discussed later in this section.

Table 5 summarizes the heat pipe design used in this simulation; all parameters are taken or derived from the experiment, with the exception of the permeability, which was not reported in [35]. The experiment estimated the power input to the evaporator from the "fuel tubes" based on a lumped energy balance and measured thermocouple values. This power is denoted by " $\dot{Q}_{\text {evap,FT, }}$, Experiment" in Figure 31 . This estimated power input was used as the input power to the evaporator in the Sockeye simulation by linearly interpolating between experimental points, which is denoted by " $\dot{Q}_{\text {evap,FT }}$, Sockeye." Also in Figure 31 is the experimental estimation of the radiative power from the condenser region and exposed evaporator region, denoted by " $-\dot{Q}_{\text {rad,nonpool }}$, Experiment"; as Reid notes, this does not account for the radiative losses from the condenser pool since there were no thermocouples in that region. For the Sockeye simulation, radiative boundary conditions, were used:

$$
q_{b}=\epsilon \sigma F\left(T_{\infty}^{4}-T^{4}\right),
$$

where $q_{b}$ is the heat flux applied to the boundary, $\epsilon$ is the emissivitiy of the surface, $\sigma$ is the Stefan-Boltzmann constant, $F$ is the view factor, $T_{\infty}$ is the environment temperature, and $T$ is the surface temperature. In the experiment, the condenser section is fully exposed (thus having a view factor of $F=1$ ), but the evaporator section is only partially exposed due to contact with the "fuel tubes," and a view factor of $F=0.25$ is used here. The environment temperature for the radiation exchange and surface emissivity from [35] are $296 \mathrm{~K}$ and 0.4 , respectively. For this demonstration, the evaporator and condenser regions were discretized into 50 elements each, and we used a BDF2 time integration with an adaptive time step size.

Figure 31 shows, in addition to experimental power estimations and Sockeye input, the resulting Sockeye 
Table 5: SAFE-30 heat pipe description.

\begin{tabular}{lr}
\hline Parameter & Value \\
\hline Working fluid & Sodium \\
Working fluid mass, $m$ & $0.1453 \mathrm{~kg}$ \\
Wick inner diameter, $D_{\text {wick,i }}$ & $0.0174 \mathrm{~m}$ \\
Wick outer diameter, $D_{\text {wick,o }}$ & $0.0207 \mathrm{~m}$ \\
Cladding inner diameter, $D_{\text {clad,i }}$ & $0.0221 \mathrm{~m}$ \\
Cladding outer diameter, $D_{\text {clad,o }}$ & $0.0254 \mathrm{~m}$ \\
Evaporator length, $L_{\text {evap }}$ & $0.43 \mathrm{~m}$ \\
Condenser length, $L_{\text {cond }}$ & $0.77 \mathrm{~m}$ \\
Wick porosity, $\varphi$ & 0.807308741 \\
Pore radius, $R_{\text {pore }}$ & $47 \times 10^{-6} \mathrm{~m}$ \\
Wick permeability, $K$ & $10^{-10} \mathrm{~m}^{2}$ \\
\hline Orientation & Horizontal \\
\hline
\end{tabular}

radiative power losses in the condenser and evaporator sections. The set " $-\dot{Q}_{\text {cond }}$, Sockeye" shows the radiation power of the entire condenser section, including the liquid pool, and " $-\dot{Q}_{\text {rad }}$, Sockeye" additionally includes the radiation power of the exposed evaporator section. Unfortunately, neither of these have a direct experimental comparison (experimental results include evaporator but not condenser pool), but it is at least useful to verify that the total radiative power is roughly following the input power.

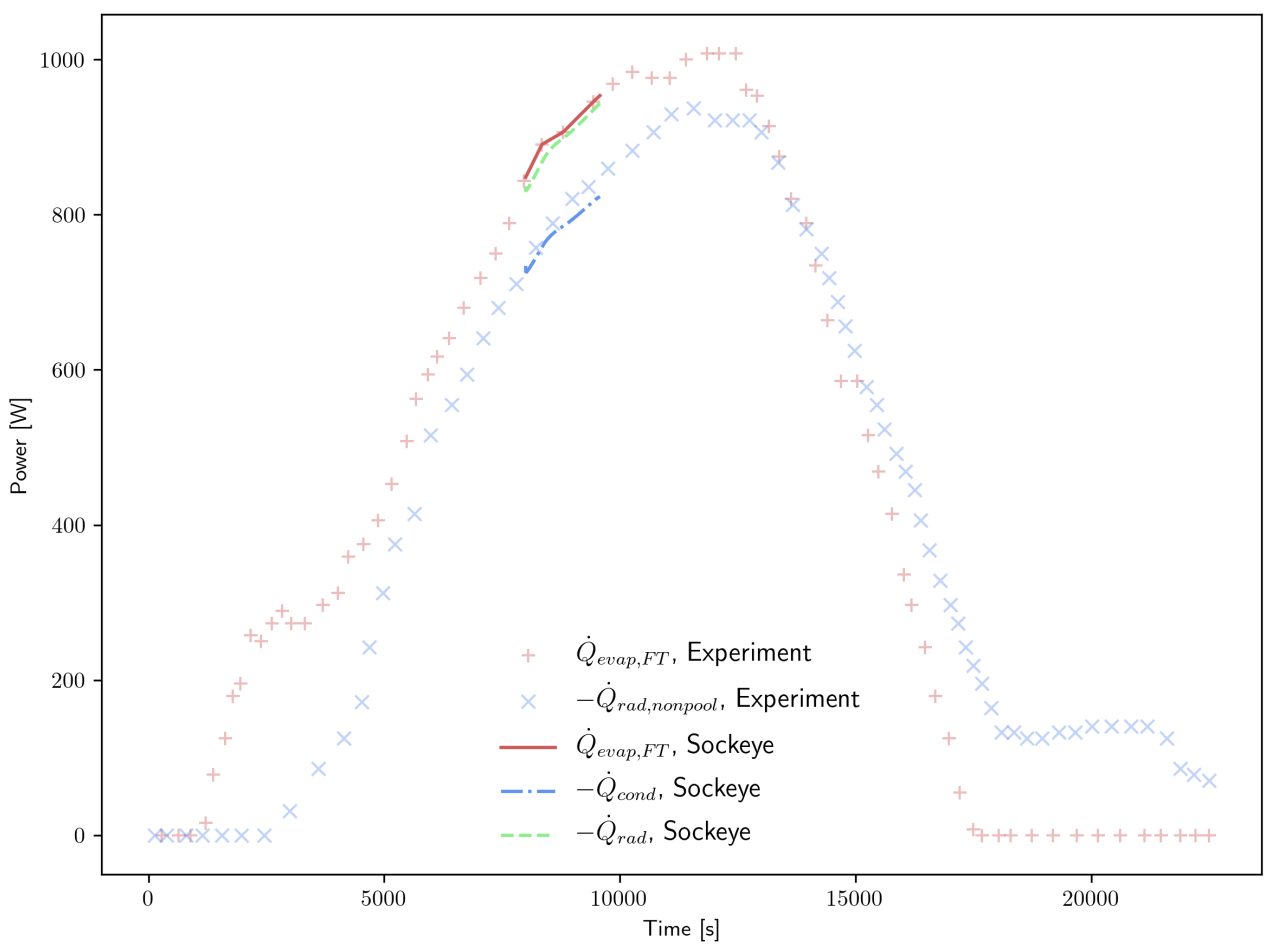

Figure 31: Comparison of experimental and numerical power transients for SAFE-30 test problem. 
Figure 32a shows the temperature transient for the five thermocouples installed on the heat pipe. These thermocouple locations are given in Table 6. Figure $32 \mathrm{~b}$ shows a zoom view for the simulated time period with error bars for the experimental data points. For Type $\mathrm{C}$ thermocouples, the error limits are the greater of $4.5{ }^{\circ} \mathrm{C}$ for temperatures up to $425{ }^{\circ} \mathrm{C}$ and $1 \%$ for temperatures up to $2320{ }^{\circ} \mathrm{C}$ [36]; thus, the error bars in the considered temperature range correspond to $1 \%$. The Sockeye solution stays within all of these error bars. Both the power and and temperature transients are strongly dependent on the modeling of boundary conditions on the cladding as well as the total thermal capacity of the heat pipe. Future work is planned to improve upon the simulation setup used here by not using experimental estimations for evaporator power input but instead using the input electric power to the heat cartridges and then perform a lumped radiation transfer between heat cartridges, fuel tubes, the heat pipe, and the environment, similar to what is done in [8].

Table 6: SAFE-30 thermocouple locations.

\begin{tabular}{lr}
\hline Name & Distance from Evaporator Endcap \\
\hline TC1 & $0.216 \mathrm{~m}$ \\
TC2 & $0.508 \mathrm{~m}$ \\
TC3 & $0.711 \mathrm{~m}$ \\
TC4 & $0.914 \mathrm{~m}$ \\
TC5 & $1.09 \mathrm{~m}$ \\
\hline
\end{tabular}

Figure 33 shows the temperature profiles at the final time, and the heat pipe operates nearly isothermally except for the liquid condenser pool, which attains a much lower temperature because its only source of heat is through condensation at its axial surface, while liquid in the rest of the heat pipe has a much greater surface-to-volume ratio. Figure 34 shows the final temperature profile in the heat pipe cladding, with the bottom edge corresponding to the inner cladding surface. The scale's lower bound was increased to exclude some of the lower temperatures in the pool region to give the radial temperature gradient more visibility.

Figures 35a and 35 show a full view and zoom view, respectively, of the computed void fraction profile. The full view shows that for most of the heat pipe, the liquid-vapor interface is near the inner surface of the wick, and the zoom view shows that the interface is nearly flat. Figure $35 \mathrm{a}$ shows that, in the final $10 \mathrm{~cm}$, a liquid condenser pool $\left(\alpha_{v} \approx 0\right)$ formed. The formation of this liquid condenser pool is the reason for the simulation failure shortly after $t=9,575 \mathrm{~s}$. Sockeye currently is not numerically robust in the limit $\alpha_{v} \rightarrow 0$; phase disappearance treatment is a high-priority item of future work. Heat pipes are commonly designed with more fluid than the annulus and wick can accommodate at a typical operating temperature, and this excess liquid volume grows with thermal expansion.

Lastly, the final solutions for velocity and pressure are presented. Velocity profiles are shown in Figure 36 and follow the trends suggested by Equation (68); that is, the vapor velocity increases where there is heating and decreases where there is cooling, with liquid velocity doing the opposite. In this case, there is a condenser pool, so the liquid velocity is zero in this region. Note that vapor solutions are not plotted where no vapor exists. The pressure profiles are shown in Figure 37. The vapor pressure features a drop over the evaporator region due to acceleration and gradual pressure recovery in the condenser; however, unlike for the test problem in Section 5.1, there is no sharp pressure drop between the evaporator and condenser. This is due to a much more gradual temperature drop between the regions, which is owed to the intermediate cladding layer and solution-dependent condenser boundary conditions. The liquid pressure profile does show a significant pressure drop between the condenser and evaporator since the liquid phase has a sharper temperature gradient there. The liquid pressure drop due to porous losses is small in comparison. 


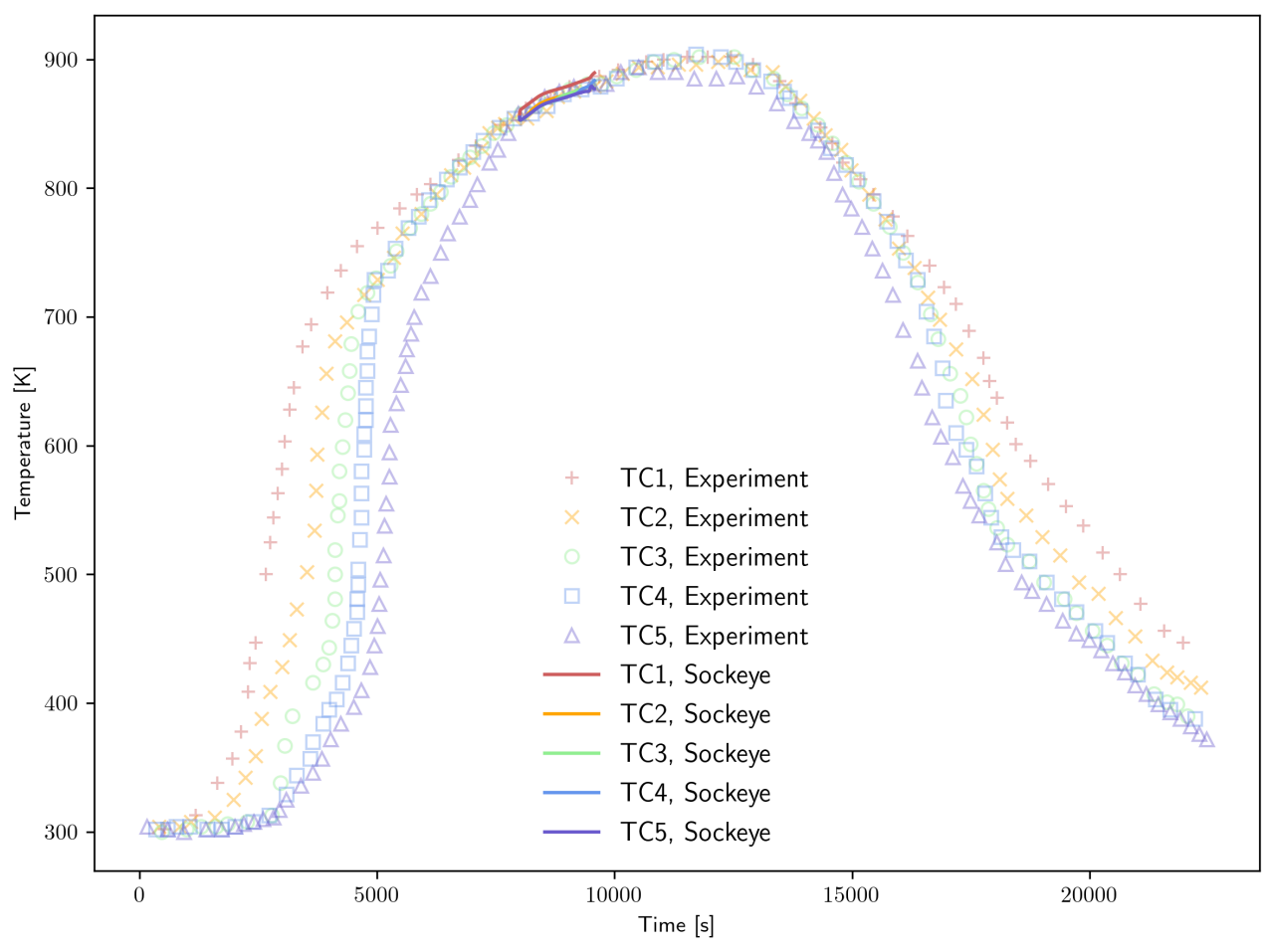

(a) Full experimental time range.

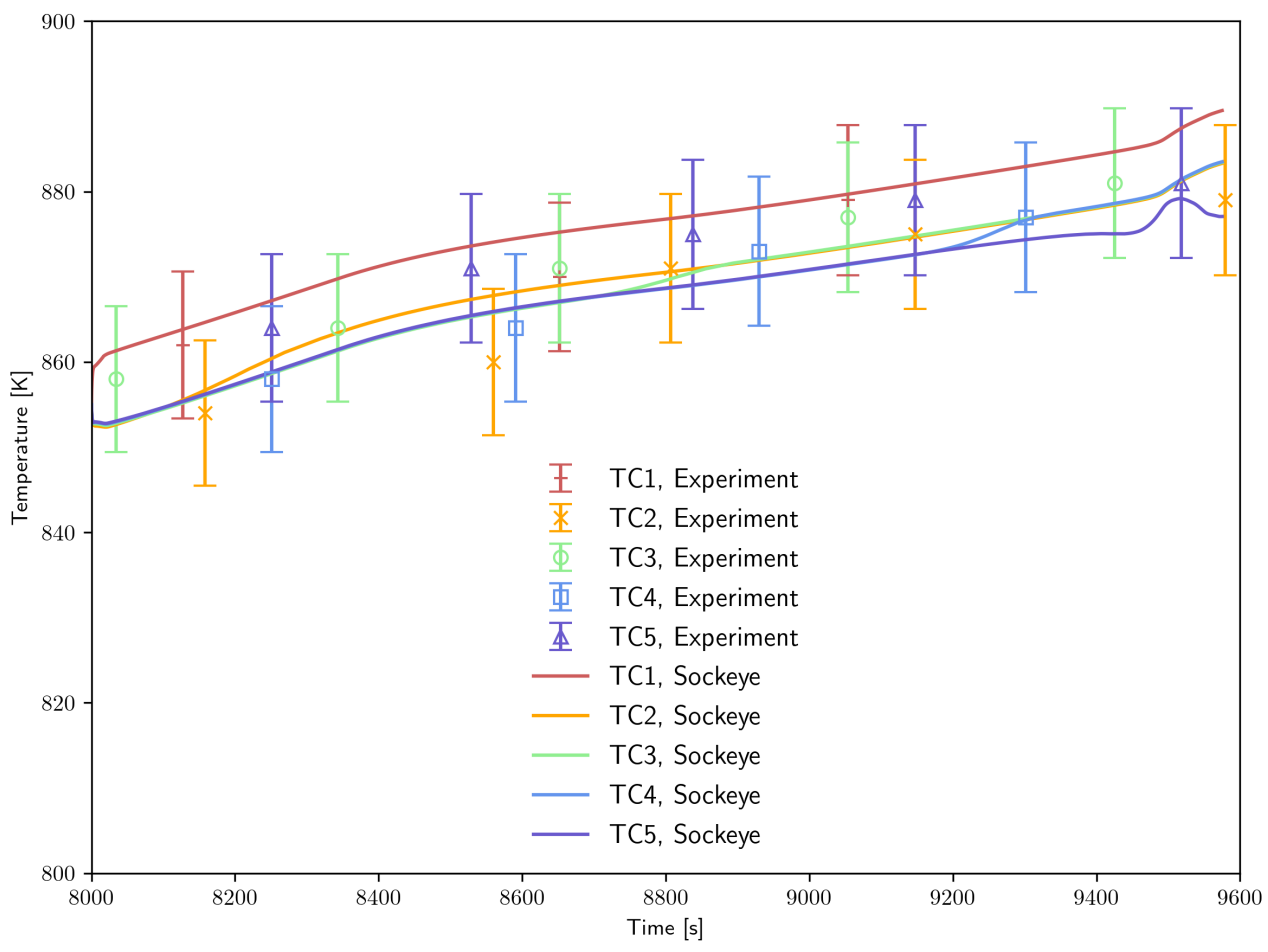

(b) Zoom to simulated time range.

Figure 32: Comparison of experimental thermocouple values against temperature solution values at thermocouple positions for SAFE-30 test problem. 


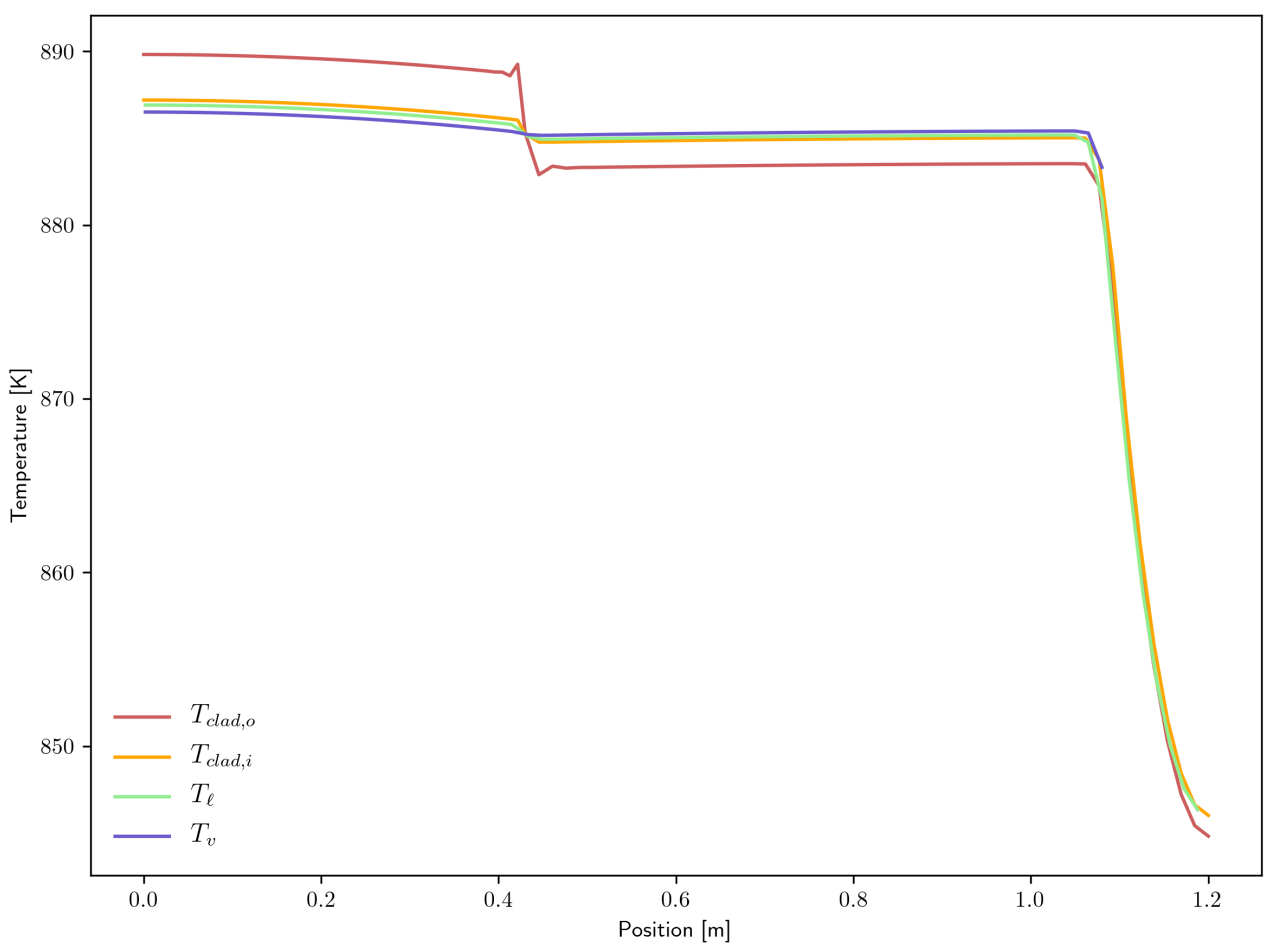

Figure 33: Final temperature profiles for SAFE-30 test problem.
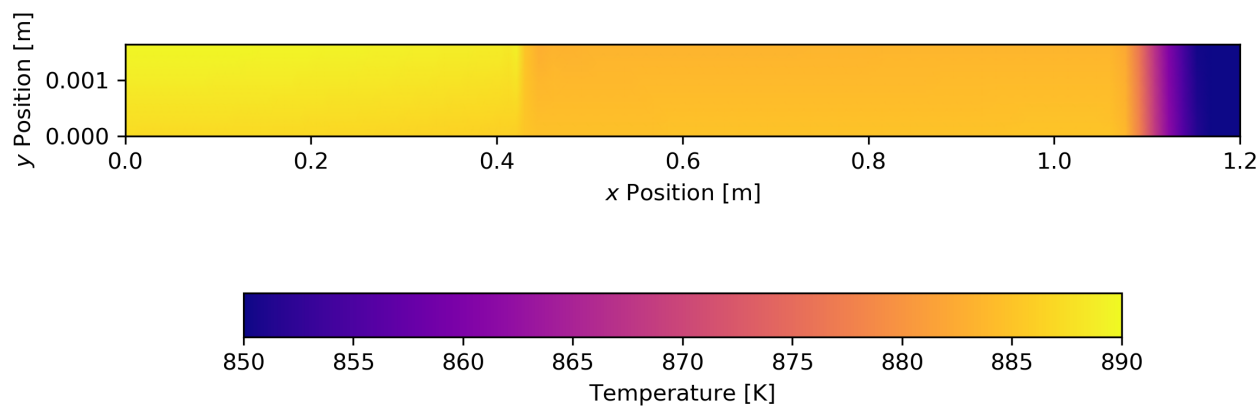

Figure 34: Final cladding temperature profile for SAFE-30 test problem. 


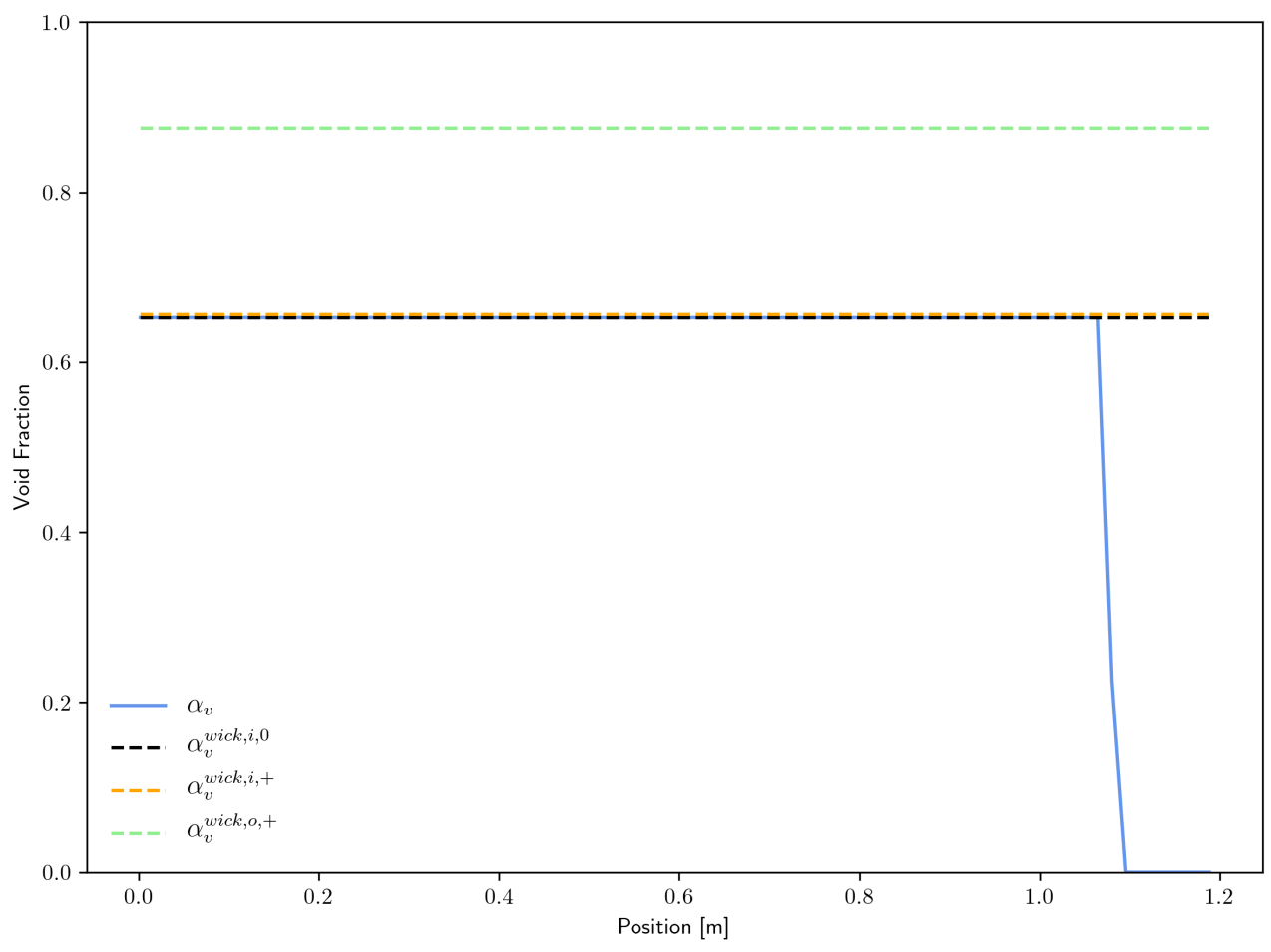

(a) Full range.

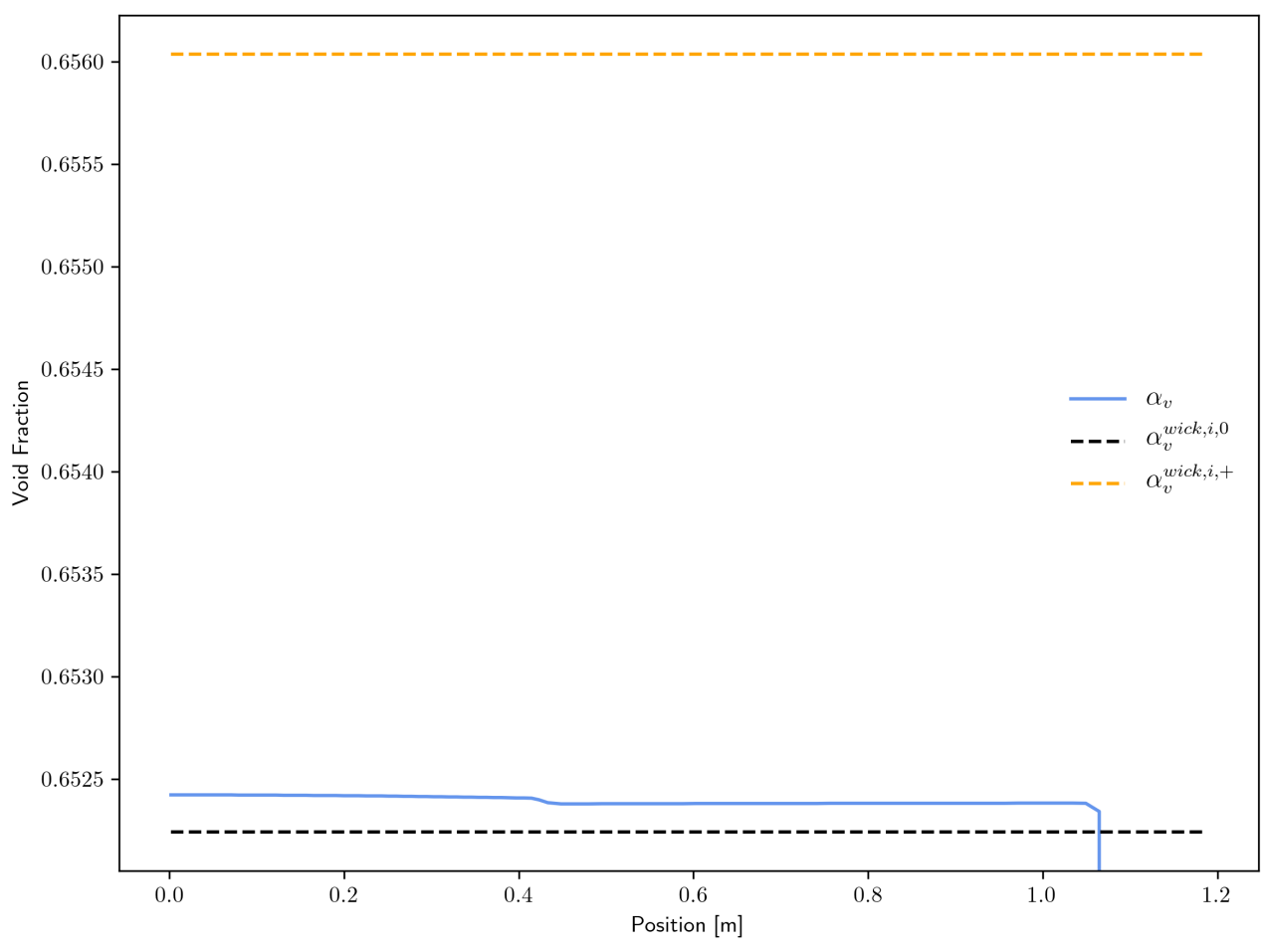

(b) Zoom about $\alpha_{v}=\alpha_{v}^{\text {wick,i, } 0}$.

Figure 35: Final void fraction solutions for SAFE-30 test problem. 


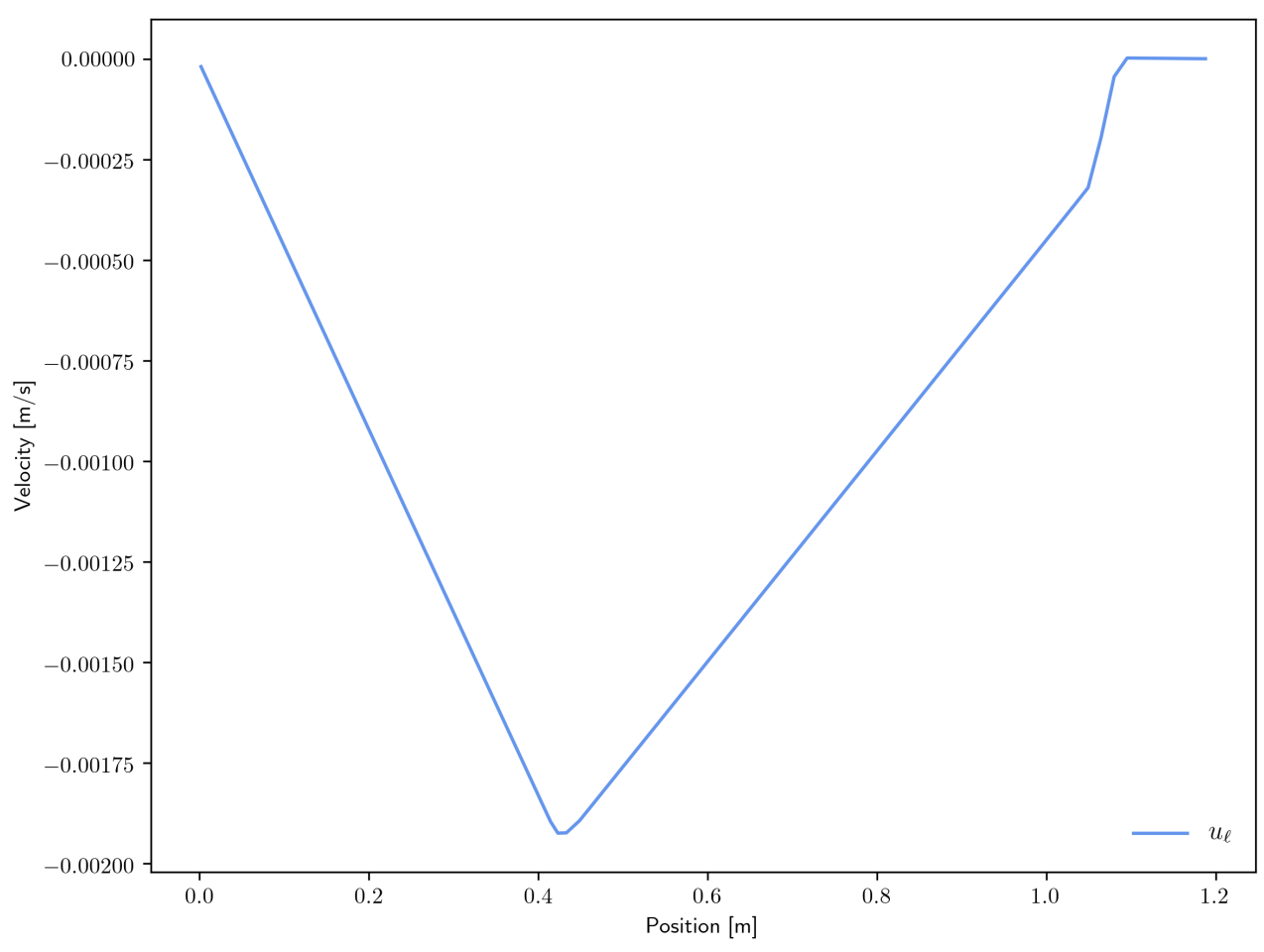

(a) Final liquid velocity solution for SAFE-30 test problem.

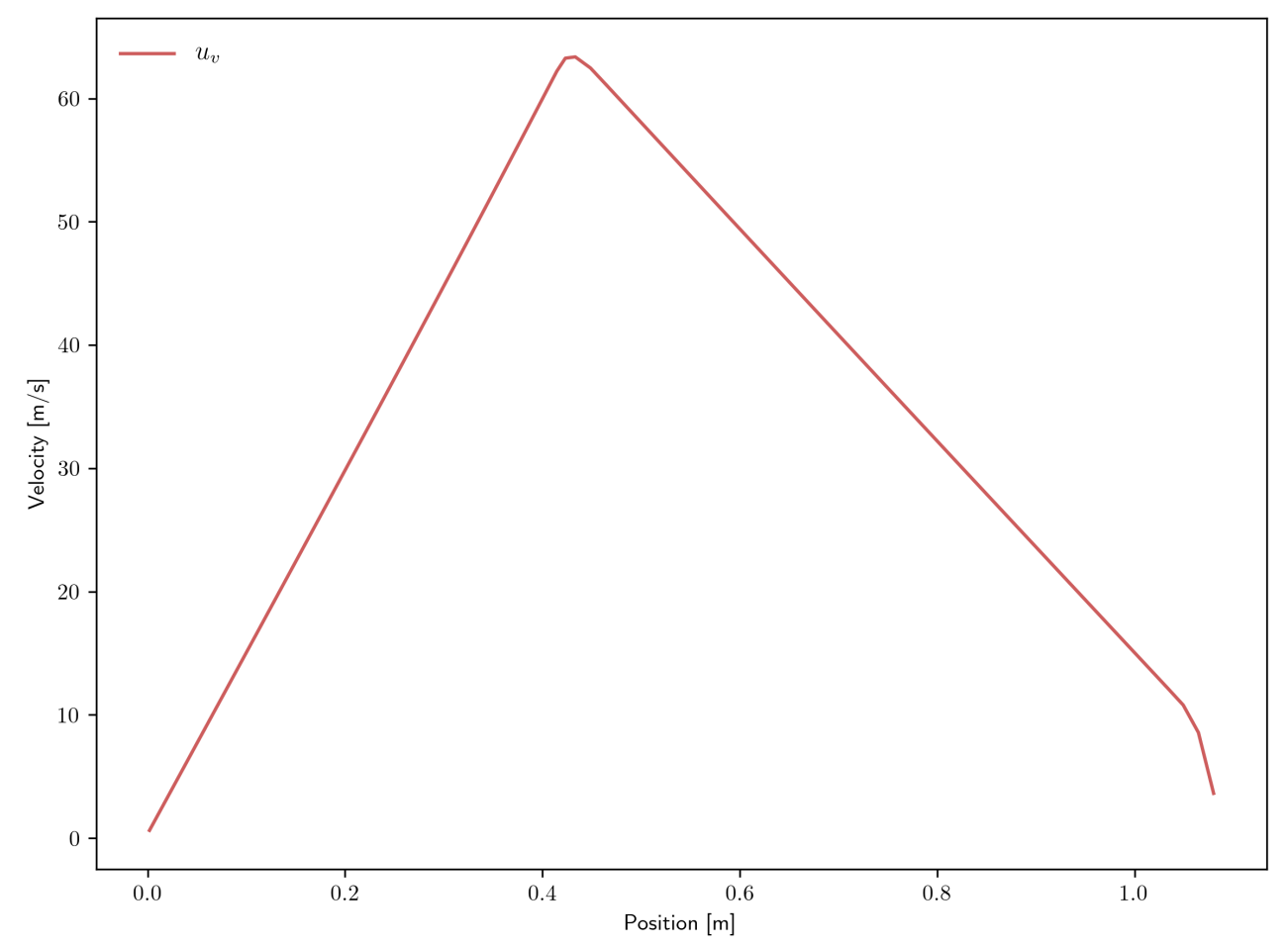

(b) Final vapor velocity solution for SAFE-30 test problem.

Figure 36: Final velocity solutions for SAFE-30 test problem. 


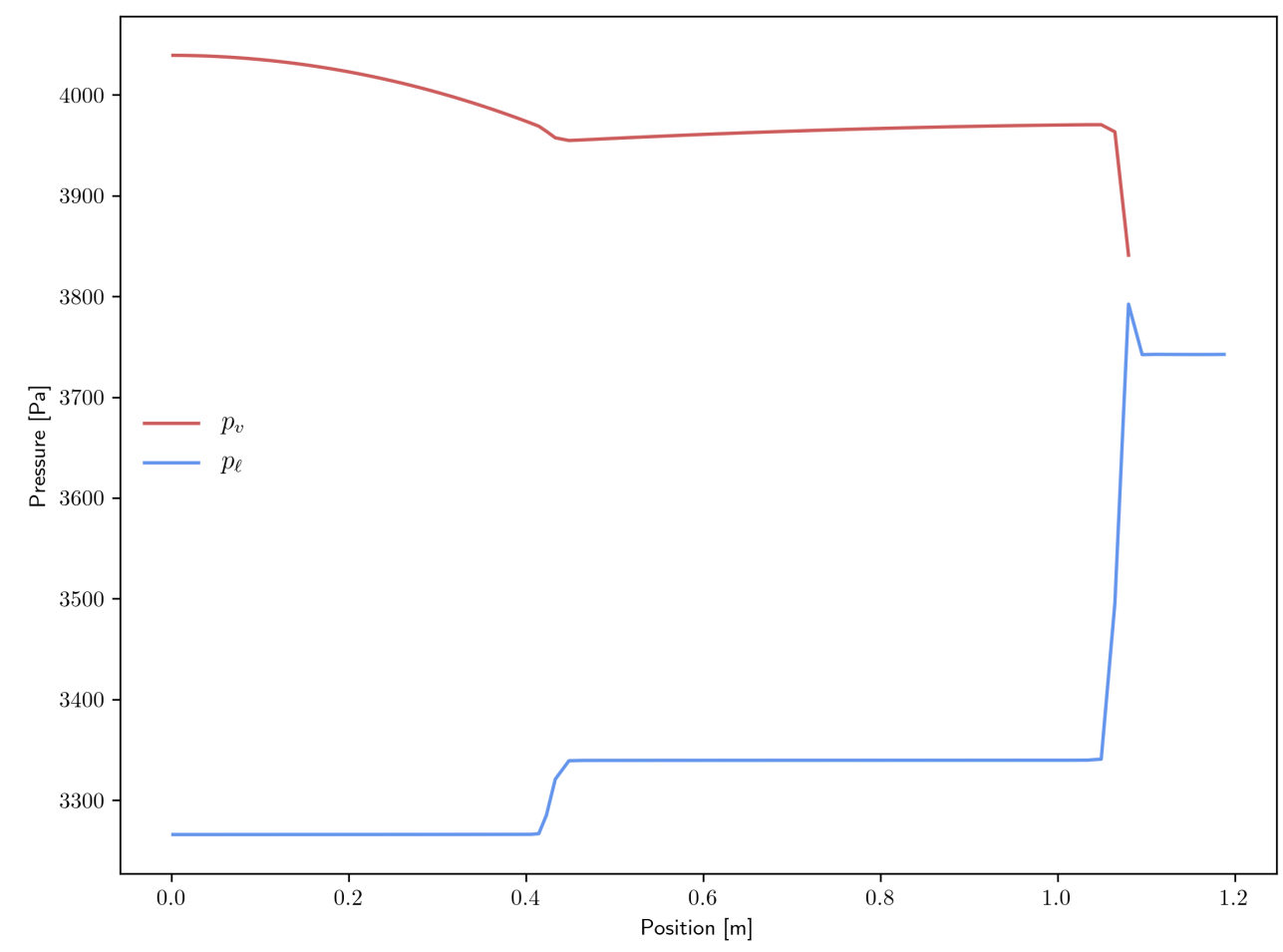

Figure 37: Final pressure solutions for SAFE-30 test problem.

\section{CONCLUSIONS}

This report discussed a multitude of improvements made to Sockeye, including new capabilities, model improvements, user documentation and examples, and verification and validation. These improvements were all conceived as a result of interaction with key stakeholders, both through their user experience and discussions about future modeling requirements. These interactions yielded advancements that were not achievable by the development team alone, as customers provided valuable insights and considerations regarding heat pipe modeling. For this reason, this relationship with stakeholders is expected to continue throughout the lifetime of the project.

New capabilities have significantly increased modeling flexibility and provided tools for complementary analyses that aid with design exploration. With the working fluid distribution initial condition ability, users can match the working fluid inventory to their heat pipe design and start simulations with realistic distributions. The ability to vary permeability allows for the simulation of the changing flow resistance associated with the long-term corrosion and degradation of the wick. The ability to vary the meniscus angle limits allows temperature-dependent capillary contact to be modeled realistically, which is important for modeling heat pipes in their lower temperature range. An alternative heat pipe model provides users with a simple, robust model that can accurately predict thermal transients when within a heat pipe's operational limits. Design utilities provide users the ability to explore the heat pipe design space without requiring simulation.

Modeling improvements have made significant strides in the accuracy of key heat pipe phenomena. Capillary pressure and pressure losses both largely determine the prediction of the capillary limit, which is arguably the most important consideration in heat pipe design. The ability to specify the capillary pressure 
relationship vs. wick saturation enables wick-specific experimental data to provide much greater accuracy than generic, rough, theoretical approximations. Wall heat transfer was also significantly improved to leverage available closures rather than rely completely on user-provided constant values and to also allow the process of wall boiling, which is necessary for capturing the boiling limit in heat pipes.

User training and documentation improvements have included numerous user examples and additions to the theory manual. Users now have examples showing how they can supply their own friction closures, use each of the different heat transfer types, and reproduce the sonic and capillary limits. Additionally, these examples laid the groundwork for the user training documentation. The theory manual now includes all of the details of the spatial discretization, which allows users to interpret details of the spatial solution.

Verification and validation tests were executed, verifying spatial convergence rates and correct steadystate velocity and pressure solutions. Validation was performed against some experimental heat pipe data from the SAFE-30 heat pipe module test and indicated a close match between the numerical temperature solution and experimental data. However, to model the full transient, a significant amount of work needs to be performed.

An extensive amount of future work was identified from this work and is discussed in Section 6.1.

\subsection{Future Work}

Future work identified from this work is extensive and includes model improvements, user documentation, and verification and validation.

As new microreactor heat pipe designs become revealed and evolve, the scope of Sockeye's heat pipe model assumptions expands. For example, a number of heterogeneous wick designs have been proposed, where, for example, a multilayer screen mesh wick can have layers of varying mesh size. These designs offer a solution to the tradeoff between heat capacity and capillary pressure when choosing a mesh size in a homogeneous wick. To accurately predict the capillary limit, users will need the ability to input radially varying wick properties.

The accurate simulation of long-term wick degradation requires a lot more work. In addition to the permeability flexibility introduced by this work, other wick properties change, such as porosity and even capillary contact. These effects are currently not well understood, and relevant experimental data is scarce, but this is an important consideration in long-term heat pipe performance.

Numerous potential improvements were outlined for the alternative heat pipe model based on heat conduction. One major improvement would be the way in which operational limits are handled. Instead of rescaling boundary conditions, the operational limits can be modeled on an individual basis as appropriate. For example, limits leading to dryout can actually change the thermal properties in the wick-annulus region to those of vapor to more mechanistically model the resulting thermal transient, and sonic and viscous limits can be modeled by cleverly modifying the thermal conductivity in the vapor core region. Another major improvement would be accounting for the latent heat of fusion when performing a startup. For example, this heat can be equivalent to the heat associated with an $80-\mathrm{K}$ temperature difference, so this consideration can significantly alter the transient response during startup and shutdown. Lastly, the lengths of pools of excess working fluid and non-condensable gases can be predicted and used to achieve a much more accurate heat transfer at the condenser side.

The design utilities in this work can be extended in scope to general heating configurations, and various approximations can be improved. Additionally, other design utilities may be conceived to provide other design exploration capabilities, such as the optimization of various wick parameters or other pre-simulation 
analyses.

The comparison to the experimental data for the SAFE-30 heat pipe module test demonstrated a crucial need for robustness enhancements to the flow model in the limit of vanishing phases. This experiment featured the formation of a pool of excess working fluid at the condenser end, and numerical robustness issues prevented the simulation of the full experiment. These condenser pools are a regular occurrence in heat pipe operation and thus need to be robustly modeled. Also, accident conditions often lead to dryout, which is a disappearance of the liquid phase, so this also needs to be robust. The numerical experiment also showed that more work needs to be performed in the startup regime.

There is plenty more user documentation and training that should be created. In addition to user examples, full tutorials should be developed to teach users in a more organized, sequential fashion. These tutorials would include subjects like how and where to run Sockeye, how to use visualization tools to analyze results, how to use design utilities, and of course, how to build models. In general, the theory manual is updated in parallel with corresponding model changes and additions; however, there is a bit more work to do in this area, such as providing the details of the derivation of the governing partial differential equations.

Future work will obviously need to include more verification and validation. Apart from the verification shown in this work, most other verification will be testing individual pieces rather than the whole system of equations. This sort of unit testing occurs in parallel with code development, as code changes must be accompanied by corresponding tests. Validation, however, is an ongoing task that relies on the influx of data from experiments, a number of which are ongoing.

\section{REFERENCES}

[1] B. Zohuri, Nuclear Micro Reactors. Springer International Publishing, 2020.

[2] A. Faghri, Heat Pipe Science and Technology. Global Digital Press, second ed., 2016.

[3] D. A. Reay, P. A. Kew, and R. J. McGlen, Heat Pipes: Theory, Design and Applications. Elsevier Ltd., sixth ed., 2014.

[4] P. Sabharwall, C.-S. Lin, J. Hansel, V. Laboure, D. Andrs, W. Hoffman, S. Novascone, A. Slaughter, and R. Martineau, "Application of integrated modeling and simulation capabilities for full scale multiphysics simulation of microreactor concepts," Tech. Rep. INL/EXT-19-55159, Idaho National Laboratory, 2019.

[5] C. J. Permann, D. R. Gaston, D. Andrš, R. W. Carlsen, F. Kong, A. D. Lindsay, J. M. Miller, J. W. Peterson, A. E. Slaughter, R. H. Stogner, and R. C. Martineau, "MOOSE: Enabling massively parallel multiphysics simulation," SoftwareX, vol. 11, p. 100430, 2020.

[6] C. Matthews, V. Laboure, M. DeHart, J. Hansel, D. Andrs, Y. Wang, J. Ortensi, and R. C. Martineau, "Coupled multiphysics simulations of heat pipe microreactors using DireWolf," Nuclear Technology, vol. 117, 2021. Submitted.

[7] A. Faghri and C. Harley, "Transient lumped heat pipe analyses," Heat Recovery Systems and CHP, vol. 14, no. 4, pp. $351-363,1994$.

[8] R. S. Reid, “Heat pipe transient response approximation,” Tech. Rep. LA-UR-01-5895, Los Alamos National Laboratory, 2002. 
[9] M. J. Hall and J. M. Doster, "A sensitivity study of the effects of evaporation/condensation accomodation coefficients on transient heat pipe modeling," International Journal of Heat and Mass Transfer, vol. 33, no. 3, pp. 465-481, 1990.

[10] Y. Cao and A. Faghri, "Transient Two-Dimensional Compressible Analysis for High-Temperature Heat Pipes with Pulsed Heat Input," Numerical Heat Transfer Part A - Applications, vol. 18, pp. 483-502, Dec. 1990.

[11] Y. Cao and A. Faghri, "A numerical analysis of high-temperature heat pipe startup from the frozen state," Journal of Heat Transfer, vol. 115, no. 247, 1993.

[12] J.-M. Tournier and M. S. El-Genk, "A heat pipe transient analysis model," International Journal of Heat and Mass Transfer, vol. 37, no. 4, pp. 753-762, 1994.

[13] "eVinci TM micro reactor." https://www.westinghousenuclear.com/new-plants/ evinci-micro-reactor. Accessed: 2021-01-19.

[14] K. Panda, I. Dulera, and A. Basak, "Numerical simulation of high temperature sodium heat pipe for passive heat removal in nuclear reactors," Nuclear Engineering and Design, vol. 323, 042017.

[15] M. Ma, W. Liang, S. Wang, Q. Xie, D. Qian, Z. Bai, T. Zhang, and R. Zhang, "A pure-conduction transient model for heat pipes via derivation of a pseudo wick thermal conductivity," International Journal of Heat and Mass Transfer, vol. 149, p. 119122, 2020.

[16] J. H. Rosenfeld, I. E. Locci, J. L. Sanzi, D. R. Hull, and S. M. Geng, "Post-test analysis of a 10year sodium heat pipe life test," Tech. Rep. NASA/TM2011-217206, National Aeronautics and Space Administration, December 2011.

[17] J. E. Hansel, R. A. Berry, D. Andrš, and R. C. Martineau, "Sockeye theory manual," Tech. Rep. INL/EXT-19-54395, Idaho National Laboratory, March 2020.

[18] M. Bader and C. A. Busse, "Wetting by sodium at high temperatures in pure vapour atmosphere," Journal of Nuclear Materials, vol. 67, pp. 295-300, 1977.

[19] Y. Cao and A. Faghri, "Closed-Form Analytical Solutions of High-Temperature Heat Pipe Startup and Frozen Startup Limitation,” Journal of Heat Transfer, vol. 114, pp. 1028-1035, 111992.

[20] T. J. Dickinson, "Performance analysis of a liquid metal heat pipe space shuttle experiment," Master's thesis, Air Force Institute of Technology, 1996.

[21] J. Hansel, D. Andrs, R. Berry, and R. Martineau, "Sockeye: A 1-D heat pipe modeling tool," Tech. Rep. INL/EXT-19-55742, Idaho National Laboratory, September 2019.

[22] M. C. Leverett, "Capillary pressure in porous solids," in Transactions of the AIME, vol. 142, pp. 152-169, Society of Petroleum Engineers, December 1941.

[23] R. A. Moss and A. J. Kelly, "Neutron radiographic study of limiting planar heat pipe performance," International Journal of Heat and Mass Transfer, vol. 13, no. 3, pp. 491 - 502, 1970.

[24] L. C. Chow and J. H. Ambrose, "Pulse mitigation and heat transfer enhancement techniques. vol. 5 transient liquid movement in heat pipe wicks," Tech. Rep. WL-TR-92-2099, Univerity of Kentucky, August 1992. 
[25] J. Charles C. Roberts, A probabilistic approach to the design of heat pipes. $\mathrm{PhD}$ thesis, The University of New Mexico, December 1972.

[26] N. M. Natarajan and S. M. Lakshmanan, "Laminar flow through annuli: Analytical method of pressure drop," Indian Chemical Engineer, vol. 5, no. 3, pp. 50-53, 1973.

[27] E. Skupinski, J. Tortel, and L. Vautrey, "Determination of convective coefficient for a sodium-potassium alloy in a circular tube," International Journal of Heat and Mass Transfer, vol. 8, pp. 937 - 951, 1965.

[28] M. Shah, "A survey of experimental heat transfer data for nucleate pool boiling of liquid metals and a new correlation," International Journal of Heat and Fluid Flow, vol. 13, no. 4, pp. 370 - 379, 1992.

[29] D. Furfaro and R. Saurel, "A simple HLLC-type Riemann solver for compressible non-equilibrium two-phase flows," Computers \& Fluids, vol. 111, pp. 159-178, 2015.

[30] J. E. Hansel, R. A. Berry, D. Andrs, M. S. Kunick, and R. C. Martineau, "Sockeye: A 1-D, two-phase, compressible flow heat pipe application," Nuclear Technology, vol. 117, 2021. Accepted.

[31] T. Cotter, "Theory of heat pipes," Tech. Rep. LA-3246-MS, Los Alamos National Laboratory, 1965.

[32] S. Gottlieb, "On high order strong stability preserving runge-kutta and multi step time discretizations," Journal of Scientific Computing, vol. 25, November 2005.

[33] R. LeVeque, L. J, and D. Crighton, Finite Volume Methods for Hyperbolic Problems. Cambridge Texts in Applied Mathematics, Cambridge University Press, 2002.

[34] E. Toro, Riemann Solvers and Numerical Methods for Fluid Dynamics: A Practical Introduction. Springer Berlin Heidelberg, 2009.

[35] R. S. Reid, J. T. Sena, and A. L. Martinez, "Sodium heat pipe module test for the SAFE-30 reactor prototype," in AIP Conference Proceedings, vol. 552, pp. 869-874, 2001.

[36] G. W. Burns, M. G. Scroger, G. F. Strouse, M. C. Croarkin, and W. F. Guthrie, "Temperatureelectromotive force reference functions and tables for the letter-designated thermocouple types based on the ITS-90," Tech. Rep. Monograph 175, National Institute of Standards and Technology, 1993. 